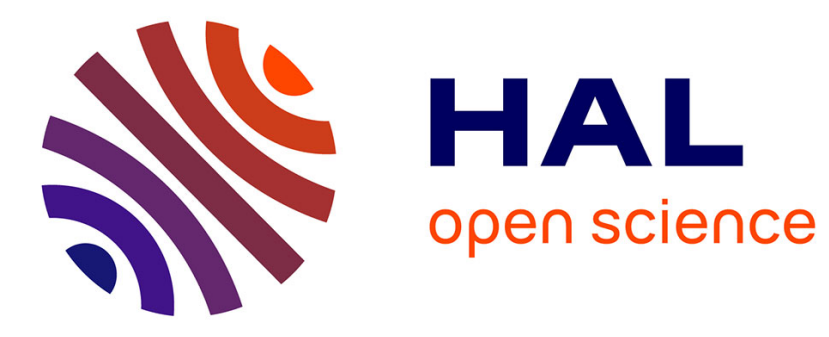

\title{
Basics of Micro/Nano Fluidics and Biology
}

Olivier Français, Morgan Madec, Norbert Dumas, Denis Funfschilling,
Wilfried Uhring

\section{To cite this version:}

Olivier Français, Morgan Madec, Norbert Dumas, Denis Funfschilling, Wilfried Uhring. Basics of Micro/Nano Fluidics and Biology. Engineering of micro/nano bio systems: fundamentals and applications, pp.7-87, 2020, Microtechnology and MEMS, 10.1007/978-981-13-6549-2_2 . hal-02404718

\section{HAL Id: hal-02404718 https://hal.science/hal-02404718}

Submitted on 11 Dec 2019

HAL is a multi-disciplinary open access archive for the deposit and dissemination of scientific research documents, whether they are published or not. The documents may come from teaching and research institutions in France or abroad, or from public or private research centers.
L'archive ouverte pluridisciplinaire HAL, est destinée au dépôt et à la diffusion de documents scientifiques de niveau recherche, publiés ou non, émanant des établissements d'enseignement et de recherche français ou étrangers, des laboratoires publics ou privés. 


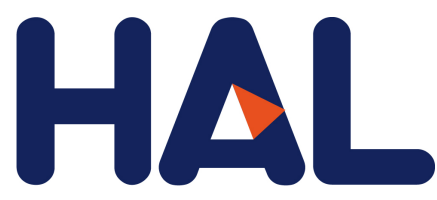

archives-ouvertes

\title{
Basics of Micro/Nano Fluidics and Biology
}

\author{
Olivier Français, Morgan Madec, Norbert Dumas, Denis Funfschilling, \\ Wilfried Uhring
}

\section{To cite this version:}

Olivier Français, Morgan Madec, Norbert Dumas, Denis Funfschilling, Wilfried Uhring. Basics of Micro/Nano Fluidics and Biology. Engineering of micro/nano bio systems: fundamentals and applications, pp.7-87, 2020, Microtechnology and MEMS, 10.1007/978-981-13-6549-2_2 . hal-02404718

\section{HAL Id: hal-02404718 \\ https://hal.archives-ouvertes.fr/hal-02404718}

Submitted on 11 Dec 2019

HAL is a multi-disciplinary open access archive for the deposit and dissemination of scientific research documents, whether they are published or not. The documents may come from teaching and research institutions in France or abroad, or from public or private research centers.
L'archive ouverte pluridisciplinaire HAL, est destinée au dépôt et à la diffusion de documents scientifiques de niveau recherche, publiés ou non, émanant des établissements d'enseignement et de recherche français ou étrangers, des laboratoires publics ou privés. 


\section{Olivier Français ,Morgan Madec , Norbert Dumas , Denis Funfschilling , Wilfried Uhring}

\section{Abstract}

Mastering the basics of theoretical and experimental aspects of fluidics and biology is an obvious requirement for the design and evaluation of micro/nano biosystems. This chapter provides a review of the phyics of micro/nano fluidics systems as well as basics of biochemistry and biological functions. It also gives detailed information on common experimental techniques used to characterize fluidic and biological systems as well as tyical examples of application.

\subsection{Introduction}

Micro- and nano-biosystems are, by nature, multidisciplinary systems involving both the common fields of engineering (such as electronics, mechanics, thermal physics, etc) but also related domains such as biology and or fluidics. It is almost impossible to gather all the knowledge and know-how required for the development of such system within the same person or the same team. Thus, projects around biosystems often involve several teams from several area of expertise. In this context, the biggest challenge which come forward before technological locks, is the ability to communicate between the different actors of the project and understand the issues of each other.

For this purpose, this chapter aims at giving the reader the theoretical background in the domain of fluidics and biology and detailed information on some experimental techniques used for micro/nnao biosystems assessment. It is composed of brief introductions on different concepts on which the reader can rely in order to understand the other chapters of this book. Extra bibliographic resources are explicitly suggested to the reader throughout the chapter for an in-depth learning of specific aspects that are only overviewed here. The chapter is divided in four parts.

The first section deals with the physical concepts related to fluidics phenomenon at micro and nanoscale. The impact of miniaturization on standard hydrodynamics laws (Navier-Stockes equation, Poiseuille flow, convection and diffusion in microfluidics channels) are discussed in the four main domains of microfluidics, i.e. the continuous flow (lab-on-chip), the centrifugal flow (lab-on-disc), the digital flow (droplet microfluidics) and the capillary flow (paper-based microfluidics).

The second section is an introduction to biology and biomolecular interactions. It starts with a brief reminder on biochemistry (atoms, molecules, functional groups, proteins). Then, the relationship between the structures of the proteins and the associated biological functions is demystified. In particular, the role of DNA, RNA, enzymes and antibodies are explained. Metabolic pathways and gene regulatory networks, which realize biological functions are also discussed. Finally, mathematical tools that are commonly used to model and simulate such systems are also presented.

The two last parts are focused on measurement techniques, and more specifically the micro-particle image velocimetry technique (MicroPIV) and some ex-situ characterization techniques mostly based on optical properties. Standard PIV techniques as well as the consequences of the scale down to microPIV are discussed. First, the properties of fluids at microscopic level (density, viscosity, surface tension, interfacial tension, contact angle) are reminded. Then, experimental aspects and the main applications of microPIV (e.g. droplet formation, blood flow measurement, wall shear stress measurement, rheology, etc) are described. The ex-situ characterization techniques considered are colorimetry and fluorescence spectroscopy. For both, the physical principles, the experimental set-up, the characteristics of elements that compose the set-ups and their applications are described.

\subsection{Microfluidics and micro and nanoscale (Olivier Français)}

Microfluidic deals with the flow study at microscale and its implementation within micro-channel networks. The applications concern a large number of industrial domains such food sector, biotechnologies or 
chemical analysis with the advantages of small volumes and well-controlled manipulation. Microfluidic is at a crossroad between biology, physic, chemistry and engineering. It benefits from progress in microelectronics for its achievement and physical effects that occur at this scale with the size reduction. Nowadays, microfluidic can be seen as a toolbox for scientific research [1] but also for industrial engineering [2].

Concerning the domains associated, the microfluidic world can be divided into four principles (Fig. 2.2.1): i) continuous flow [3] (case of classical microfluidic used in Lab On Chip where pressure driven handle the flow), ii) centrifugal flow [4] (case of Lab On Disc devices based on spinning effect), iii) digital flow [5] (case of two phase flow with droplet generation) and iiii) capillary flow [6] (associated to paper based microfluidic).

In this section, the Physics behind these four categories will be covered from a basic point of view, having in mind the impact of the miniaturization [7]. First of all, continuous hydrodynamics must be discussed taking into account the scaling factor of miniaturization. In fact, fluidic flow at micro-scale is mainly driven by pressure and viscous looses. As consequences, the Navier-Stokes equation governs the mass transport and the diffusion associated. For the diphasic flow (droplet), capillary phenomena dominate and enable stable droplet generation. With centrifugal flow, inertial effects are exploited to move liquid within microchannel embedded inside the disc. Finally, the surface tension drives the fluid front inside paper and thus induces liquid and mass transport towards analysis zone.

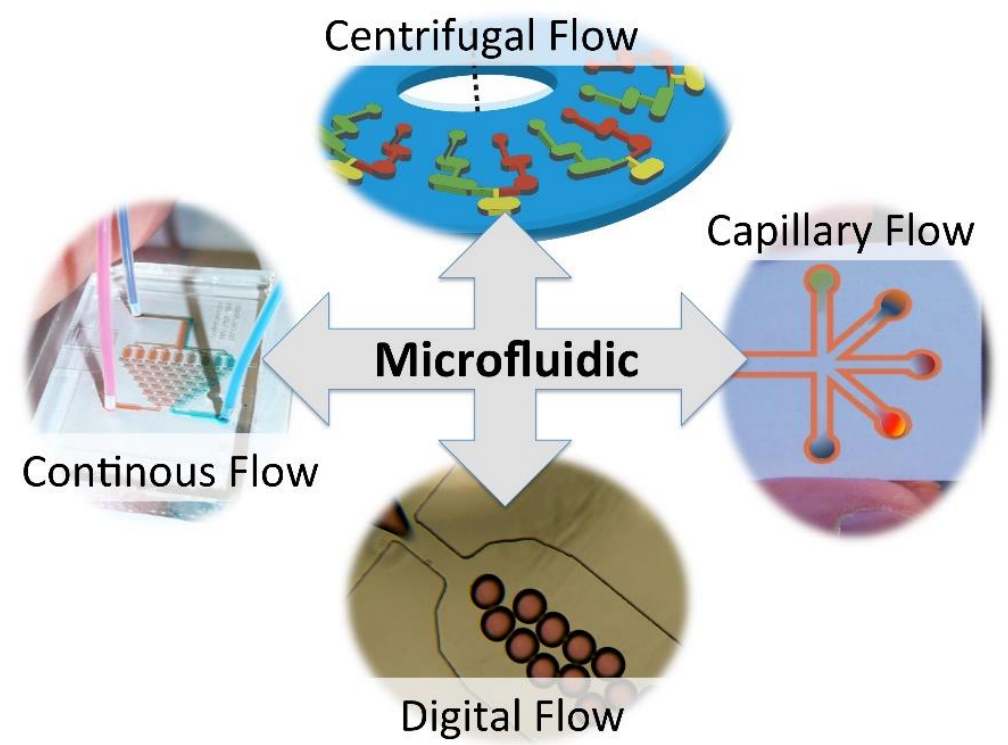

Fig. 2.1. Microfluidic devices: using physics to handle liquid at micro-scale

\subsubsection{Taking benefits from size reduction within microfluidic devices}

\subsubsection{Scaling laws}

Flow behavior in miniaturized channels can be estimated using dimensionless parameters, which indicate the predominant physical phenomena, by a ratio study. These scaling laws rule the world, define life behavior and have many applications concerning miniaturization [8]. 
As a first approach, with size reduction, volumetric effects will decrease faster compare to surface and linear effects. The classical effects of fluid physics at human scale may be completely opposite at microscale. To illustrate the scaling laws, one example is the capability for insects to walk on water compared to human that sink into it. The physic is the same for the two scales, but the consequence not... It's a matter of balance between the gravitational force $\left(\mathrm{F}_{\mathrm{g}}\right)$, which depends on mass of the object and thus depends on three power with size $\left(\mathrm{F}_{\mathrm{g}} \approx[\mathrm{s}]^{3}\right)$, and the capillary force $\left(\mathrm{F}_{\mathrm{c}}\right)$ associated to the surface tension coefficient multiplied by the contact perimeter, with a dependence of one power with size $\left(\mathrm{F}_{c} \approx[\mathrm{s}]^{1}\right)$.

Thus, at microscale the capillary force dominates compare to gravity force. The ratio between the capillary and gravity forces increases with the size reduction. It becomes greater than one at millimeter scale.

Based on this scaling approach, the predominant physics for liquid handling at microscale can be predicted and confirmed with the classical parameter values used in the field of microfluidic.

\begin{tabular}{|c|c|c|}
\hline Channel hydraulic diameter & $D_{H}$ & $10-200 \mu \mathrm{m}$ \\
\hline Average flow velocity & $V_{O}$ & $0.1-10 \mathrm{~mm} \cdot \mathrm{s}^{-1}$ \\
\hline Water dynamic viscosity $\left(20^{\circ} \mathrm{C}\right)$ & $\eta$ & $1 e^{-3} \mathrm{~Pa} \cdot \mathrm{s}$ \\
\hline Water Density & $\rho$ & $1 e^{3} \mathrm{~kg} \cdot \mathrm{m}^{-3}$ \\
\hline Surface tension $\left(20^{\circ} \mathrm{C}\right):$ & $\sigma$ & \\
- Water / Air & & $72.94 \mathrm{mN} \cdot \mathrm{m}^{-1}$ \\
- Water / Oil & & $25 \mathrm{mN} \cdot \mathrm{m}^{-1}$ \\
\hline Diffusion Coefficient in water: & $\mathrm{D}$ & \\
- Hemoglobin (Hb) & & $0.06 e^{-9} \mathrm{~m}^{2} \cdot \mathrm{s}^{-1}$ \\
- DiOxyde (O $\left.{ }_{2}\right)$ & & $2.0 e^{-9} \mathrm{~m}^{2} \cdot \mathrm{s}^{-1}$ \\
- Sodium ion (Na $\left.{ }^{+}\right)$ & & $13.3 e^{-10} \mathrm{~m}^{2} \cdot \mathrm{s}^{-1}$ \\
- Fluorescine dye & & $5.2 e^{-10} \mathrm{~m}^{2} \cdot \mathrm{s}^{-1}$ \\
- 10 nm nanoparticle & & $2.2 e^{-11} \mathrm{~m}^{2} \cdot \mathrm{s}^{-1}$ \\
\hline
\end{tabular}

Table 2.1. Typical value parameters encountered in microfluidic

The volumes involve in microfluidic are in the order of the nano-liter. A microchannel with a hydraulic diameter $D_{H}$ of $50 \mu \mathrm{m}$ and a length of 1 centimeter corresponds to a volume of 20 nano-liters. To compare, a metric droplet corresponds to $50 \mu l$, which fills $25 \mathrm{~m}$ of a channel with $D_{\mathrm{H}}=50 \mu \mathrm{m}$.

\subsubsection{Fluid physics at microscale: dimensionless parameters analysis}

At microscale, classical physical laws can be used but the predominance forces or effects must be carefully examined to well understand the principle of microfluidic device [9].

Reynolds number: $\mathbf{R}_{\mathbf{e}}$

The Reynolds number (Re) represents the ratio between fluid inertial forces and viscous forces (Eq. 2.1). Its value gives the regime of the flow. For a value below one, the regime is laminar and thus reversible with well-defined and stable flow path. Above three thousands, the regime is turbulent and gives complex pathway for the flow velocity line, which is totally instable [10].

Re is defined by the formula:

$$
R_{e}=\rho \frac{V L}{\eta} \rightarrow\left[S^{2}\right]
$$


As $L$ represents the characteristic length of the channel and $V$ is a distance over the time, the dependence with size for $\mathrm{Re}$ is a two power. It means that reducing the size by a factor ' $\mathrm{k}$ ' will impact Re value by a factor $k^{2}$ and tends to greatly reduce its value.

From values used in microfluidic (see Table 2.2.1), Re varies in the range :

$$
0.001<\operatorname{Re}<0.2
$$

Consequently, it means that the flow within microfluidic channel can be well controlled and studied with a deterministic behavior (Fig. 2.2.2). The flow is laminar; the viscous effect dominates compared to inertial effect.

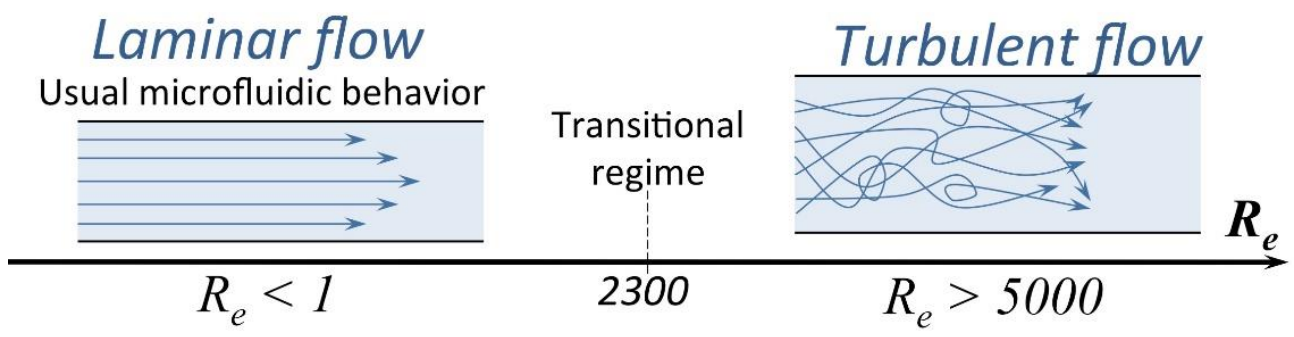

Fig. 2.2. Flow behavior in relation with the Reynolds number

Peclet number: $P_{\mathrm{e}}$

The Peclet number $\left(\mathrm{P}_{\mathrm{e}}\right)$ characterizes the nature of the mass transport by a ratio between the time of diffusion ( $t_{D}$ ) and the time of convection ( $t_{c}$ ) (Eq. 2. 3) [11]. The diffusion time, due to the Brownian's motion, is inversely related to the diffusion coefficient (D) of the molecule while the convection time is directly linked to the flow velocity $(V)$. For a distance ' $L$ ' of transport, the expression of $t_{D}$ and $t_{c}$ are:

$$
t_{D}=\frac{L^{2}}{D} \text { and } t_{c}=\frac{L}{V}
$$

It gives the expression for $\mathrm{P}_{\mathrm{e}}$ :

$$
P_{e}=\frac{t_{D}}{t_{c}}=\frac{L V}{D}
$$

For a value of $\mathrm{P}_{\mathrm{e}}$ above 1 , the flow is mainly convective and the mass transport is governed by the streamlines. Below 1, diffusion dominates and the molecules spread within the medium, the velocity having no influence on the transport, at the scale of a distance ' $\mathrm{L}$ '. As for Reynolds number, its size dependence is a two power, which tends to give low value for Pe, considering a mass transport over a distance 'L' (Eq.2.4). Combined with the laminar flow behavior, it means a straight mass transport where mixing between two mediums will not be very efficient, as only diffusion occurs.

Advantages will be the design of chemical gradient generator taking into account the diffusion law within a laminar flow [12]. Applications are towards biology (chemotaxis analysis), particle handling in chemistry [13] or study of reaction in the case of molecule synthesis [14].

\section{Capillary number: $C_{a}$}

In case of a biphasic flow, the behavior between the two liquids (usually water and oil) can be estimated using the capillary number $\left(C_{a}\right)$ [15]. $C_{a}$ is the ratio between the viscous forces and the capillary forces (Eq. 5). It is defined with the expression: 


$$
C_{a}=\frac{\eta V}{\sigma}
$$

The capillary force tends to reduce the interfacial area, in opposition the viscous force tries to extend it. At $C_{a}$ number below one, droplet will be stable and reproducible (monodisperse) with a spherical shape. The flow behavior is similar to a dripping jet. With $C_{a}$ number above one, droplets are stretched and unstable. The flow behaves with jetting shape between the two phases.

In microfluidic, as $\mathrm{C}_{\mathrm{a}}$ is directly proportional to the scale of the object, size reduction favorises low value for $C_{a}$. Usual value for $C_{a}$ are between 0.001 and 1 .

\section{Bond number:}

The Bond number (Bo) compares the gravity force over the capillary force [16]. Its formula is given by:

$$
B o=\frac{\rho l^{2} g}{\sigma}
$$

Based on this relation, the capillary length $I_{c}$ is defined when $B o=1$ :

$$
l_{c}=\sqrt{\frac{\sigma}{\rho g}} \sim 2.6 \mathrm{~mm}
$$

Thus, at microscale the capillary is dominant ( Bo $<1$ ) and can be used: i) to travel liquid (Paper based microfluidics) without real impact of the gravity or ii) to get round shape droplet with no deformation induce by their weight.

\subsubsection{Micro-hydrodynamic flow}

\subsubsection{The Navier-Stokes equation}

Hydrodynamic of liquid flow remains fluid down to several nanometers. So in classical microfluidic, the fluid is assumed to be a continuous medium. Considering an incompressible Newtonian fluid, the Navier-Stokes equation (Eq. 2.8) can be used to describe the velocity field [17]:

$$
\rho\left[\frac{\delta \vec{v}}{\delta t}+(\vec{v} \cdot \vec{\nabla}) \vec{v}\right]=-\vec{\nabla} P+\eta \Delta \vec{v}+\vec{f}_{v o l}
$$

The two left terms of the equation deals with the inertial effect, which can be neglicted within microfluidic channel at steady state. The right terms corresponds to the pressure force, the viscous force and the external volumetric forces where the gravity can be neglicted.

\subsubsection{Poiseuille flow: Stokes equation}

At steady state, taking into account the Reynold's number low value $(<<1)$ meaning that inertial term can be neglected, the Navier-Stokes equation can be reduced to its simple expression with no external force:

$$
\vec{\nabla} P=\eta \Delta \vec{v}
$$


Equation 2.9 corresponds to Poiseuille flow. It shows the balance between the pressure force and the viscous force.

\section{Case of a cylindric channel}

It can be easily solved in case of a circular channel, considering a no slipping boundary condition.

Supposing a stationary laminar flow in the $z$ direction within a channel with a radius $R_{h}$, due to symmetric simplifications the velocity profile only depends on radius direction and the pressure drop is on the ' $z$ ' direction. In these cylindric coordinates, equation (2.9) becomes:

$$
\frac{\delta P}{\delta z}=\eta \frac{1}{r}\left(\frac{\delta}{\delta r}\left(r \frac{\delta v_{r}(r)}{\delta r}\right)\right)
$$

The pressure drops linearly along the channel. For a channel length $L$, with boundaries conditions such as $p(z=0)=P_{0}$ and $P(z=L)=0$ given a pressure evolution :

$$
p(z)=-\frac{P_{o}}{L}(z-L) \rightarrow \frac{\delta P}{\delta z}=-\frac{P_{o}}{L}
$$

Supposing no slipping condition along the wall channel $(v(R)=0)$ and maximum velocity value at $\mathrm{r}=0\left(\frac{\delta v(0)}{\delta r}=0\right)$, the velocity profile expression is then:

$$
v_{r}(r)=\frac{-P_{o}}{2 \eta L}\left(\frac{r^{2}-R^{2}}{2}\right)=\frac{P_{o} R^{2}}{4 \eta L}\left(1-\frac{r^{2}}{R^{2}}\right)
$$

The velocity profile has a parabolic shape within the microchannel (2.12). The maximum velocity $v_{\text {max }}$, the average velocity $\mathrm{V}$ and the flow rate $\mathrm{Q}$ are then :

$$
\begin{gathered}
v_{\max }=\frac{P_{O} R^{2}}{4 \eta L} \text { and } V=\frac{1}{R} \int_{O}^{R} v(r) d r=\frac{2}{3} v_{\max } \\
Q=\int_{O}^{R} 2 \pi r \cdot v(r) d r=\left(\frac{\pi R^{4}}{8 \eta L}\right) P_{O}
\end{gathered}
$$

\section{Case of squeeze film flow: Hele Shaw cell}

In microfluidic, due to technical constraint, the channel section is rectangular. It can be defined by its width $w$ and height $h$ (Fig. 2.3).
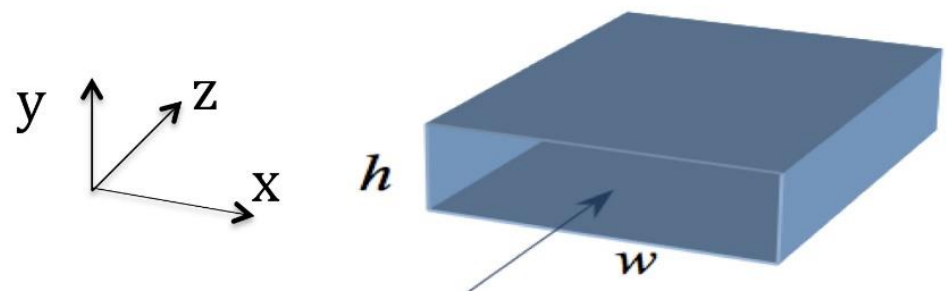

Fig. 2.3. Schematic view of a rectangular channel

The laminar flow is in the $z$ direction and the velocity profile depends mainly on the $y$ axis : assuming $\mathrm{h} \ll \mathrm{w}$, edge effects are neglected in the $\mathrm{x}$ direction. Thus, following the same methodology of resolution used for circular channel, but here in Cartesian coordinates for thin rectangular channel, the solution of the stokes equation gives : 


$$
\frac{\delta P}{\delta z}=\eta\left(\left(\frac{\delta^{2} v_{z}}{\delta^{2} y}\right)\right) \Rightarrow v_{z}=-\frac{P_{O}}{2 \eta L}\left(y^{2}-\left(\frac{h}{2}\right)^{2}\right) \text { et } v_{x}=v_{y}=0
$$

The profile obtained is parabolic (Eq. 2.15) with a maximum velocity at the middle of the channel (Fig.2.4.A). The relation between the pressure applied and the flow rate is then :

$$
P_{o}=\frac{12 \eta L}{w h^{3}} Q
$$

In case of square channel, similar calculation can be done but simple analytical calculation can be achieved. The profile is still parabolic (Fig. 2.4.B).

\section{Hydraulic resistance}

From previous calculation describing the laminar flow within circular or planar channel, the relation between the pressure drop $\Delta P$ and the flow rate $\mathrm{Q}$ (Eq. 2.2.14) is proportional (Eq. 2.2.16). This behavior is equivalent to a hydraulic resistance $R_{H}$ defined as:

$$
R_{H}=\frac{\Delta P}{Q}
$$

The value of the hydraulic resistance $R_{H}$ is defined by the channel profile (circular, square, rectangular...)

\begin{tabular}{|c|c|c|c|c|}
\hline C & Circular & Planar & Square & Rectangular \\
\hline & & & $\mathrm{h}$ & \\
\hline profile & & $\mathrm{h}$ & $\mathbf{h}$ & h \\
\hline Parameters & Radius $r$ & $h<<w$ & $h=w$ & $0.2<h / w<1$ \\
\hline $\begin{array}{l}\text { Hydraulic } \\
\text { resistance } R_{H}\end{array}$ & $\frac{8}{\pi} \eta L \frac{1}{r^{4}}$ & $12 \eta L \frac{1}{h^{3} w}$ & $28.4 \eta L \frac{1}{h^{4}}$ & $\frac{12 \eta L}{1-0.63(h / w)} \frac{1}{h^{3} w}$ \\
\hline
\end{tabular}
[18]. In Fig. 2.4.C, the $R_{H}$ expression used in microfluidic is given for four cases.

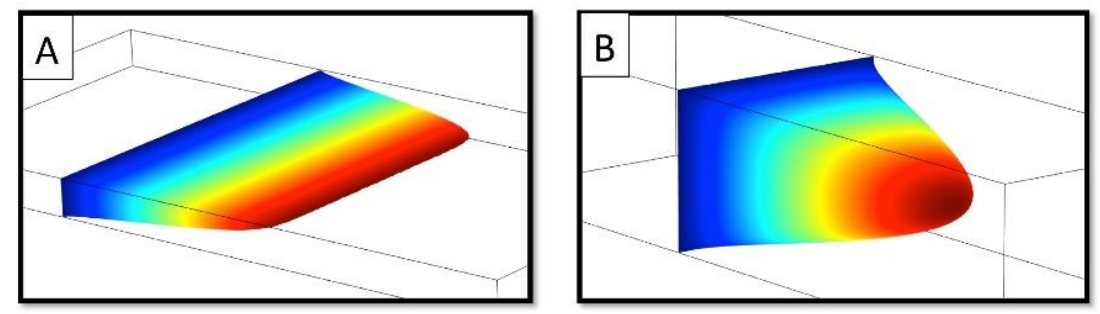

Fig. 2.4. A. Flow profile in a planar channel; B. Flow profile in a square channel; C. Hydraulic resistance expression with the channel shape

The general formula for a rectangular channel can be obtained with a serial Fourier analysis and be implemented under mathematic software if accurate results are desired:

$$
R_{H}=\frac{12 \eta L}{w h^{3}}\left[1-\frac{h}{w}\left(\frac{192}{\pi^{5}} \sum_{n=1,3,5, \ldots}^{\infty} \frac{1}{n^{5}} \tanh \left(\frac{\pi n w}{2 h}\right)\right]^{-1}\right.
$$

\section{Analogy with an electrical network}

Thus, a direct analogy of a microfluidic network can be donen with an electric network. The electrical potential is equivalent to the pressure, the current corresponds to the flow rate. In consequence, 
this analogy can simplify the design of a microfluidic network and the study of its flow behavior. The Kirchoff's law can be applied (node and mesh laws) (Fig. 2.5) [19].

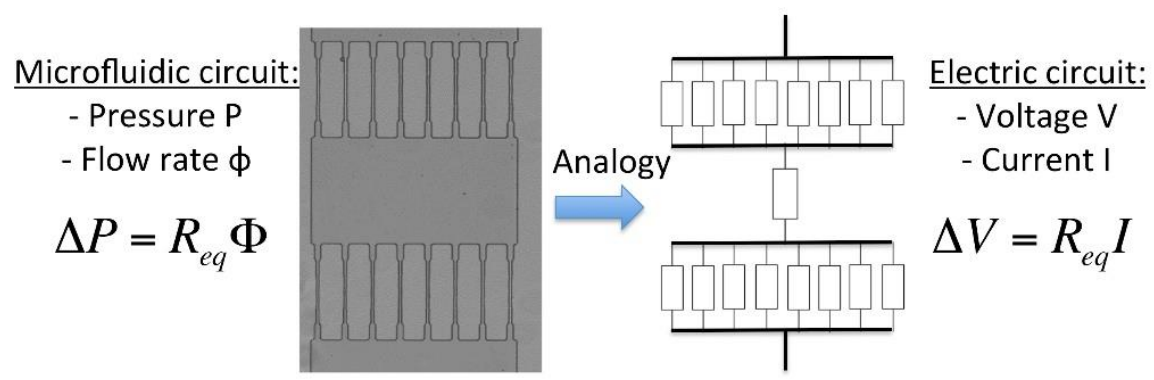

Fig. 2.5. Equivalent representation of a microfluidic network with the help of an electrical circuit

A microfluidic circuit can be analysed with an equivalent electrical schematic based on electric resistance for all pressure and flow rate distributions.

\subsubsection{Mass transport within a microfluidic channel}

\subsubsection{Convection and diffusion}

As depicted in part 2.2.1, the flow in microfluidic is generally laminar. As consequence, two miscible liquids in contact will induce a bi-dimensional flow, the absence of turbulence restrains the mixing only by diffusion (Fig. 2.6) [20].
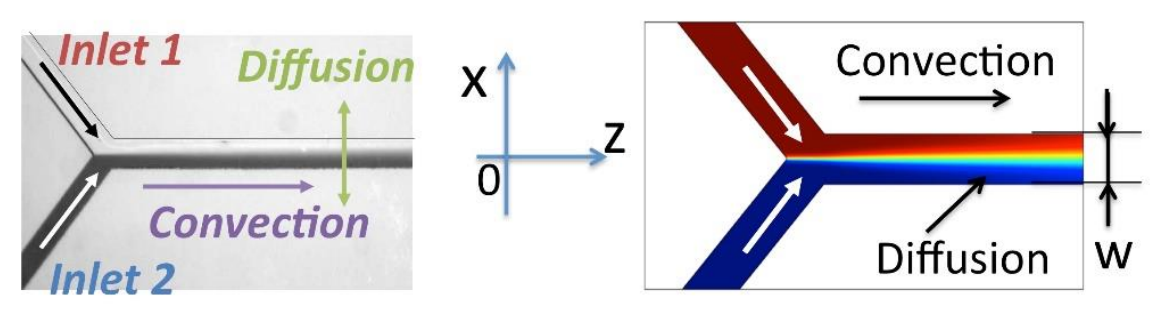

Fig. 2.6. Mass transport between two miscible liquids within a microfluidic network : convection with the flow velocity and diffusion by Brownian motion

Taking advantage of this behavior is a way to design gradient generator of chemical species within microfluidic channel for chemotaxy analysis for example [21][22]. It's also a way to study the chemical reaction kinetic between two species. In fact along the two liquids, the contact position becomes the time of reaction, which is a crucial parameter to clearly understand the evolution of a chemical reaction.

\subsubsection{Study of concentration profile}

For microfluidic, in a straight channel under flow, the mass transport will be a competition between the natural diffusion of the particle in a transverse direction of the flow (here $x$ direction) and its convection due to the velocity profile following the flow (here $z$ direction). The fick's law and the mass conservation combination give the equation governing this transport:

$$
\frac{\delta c(t)}{\delta t}=D \Delta c(t)-\vec{v} \vec{\nabla} c(t)
$$


Solving this equation needs to take into account the velocity profile to determine the concentration evolution with time and position within the microchannel. It's a complex process to find the real solution of the mixing evolution, but with some specific approximations this 3D problem can be reduced to an approximate 1D problem:

- the Peclet's number associate to the flow is assumed to be high (Pe>1) compare to the size of the channel under study.

- Having Pe $>1$, the diffusion and convection process can be isolated and studied independently.

- Diffusion occurs in the $x$ direction (transverse of the flow) and behaves like and 1D domain.

- Convection occurs in the $z$ direction with a uniform average velocity. The time of diffusion will be connected with the position in the channel with the relation : $z=V t$.

\subsubsection{Mass transport in the context of microfluidics}

Thus the mass transport for the diffusion part can be reduced to a 1D problem (Eq. 2.20), in the transverse direction compared to the flow direction:

$$
\frac{\delta c(x, t)}{\delta t}=D \frac{\delta^{2} c(x, t)}{\delta^{2} x}
$$

Starting with a concentration profile at $\mathrm{t}=0$ (corresponding to $\mathrm{z}=0$ ) :

$$
\text { For } x<0 c(x, t)=C_{o} \text { and } x>0 c(x, t)=0
$$

The profile evolution of the concentration is depicted by :

$$
c(x, t)=\frac{1}{2} C_{o}\left(1-\operatorname{erf}\left(\frac{x}{2 \sqrt{D t}}\right)\right)
$$

where erf is the Gauss error function :

$$
\operatorname{erf}(k)=\frac{2}{\sqrt{\pi}} \int_{o}^{k} \exp \left(-u^{2}\right) d u
$$

It is common to use as diffusion length scale the parameter $I_{D}$, which gives a concentration close to half the final solution:

$$
l_{D}=\sqrt{D t}
$$

Here starting from a centered profile $[\mathrm{Co}, 0]$ in $\mathrm{x}$ direction, the final solution will be a homogenous concentration of $\mathrm{Co} / 2$. To get the condition of half the final solution across the width $(w)$ of the channel, the time of diffusion can be put in relation with the length of the channel $L$ with the average velocity field $V$ (Eq. 2.24).

$$
\frac{w}{2}=\sqrt{D t} \text { with } t=\frac{V}{L}
$$

At steady state, the concentration profile with the microchannel is then :

$$
c(x, z)=\frac{1}{2} C_{o}\left(1-\operatorname{erf}\left(\frac{x \sqrt{V}}{2 \sqrt{D z}}\right)\right)
$$

This profile evolution is presented in Fig. 2.7. It shows the progressive diffusion of species along the microchannel. 


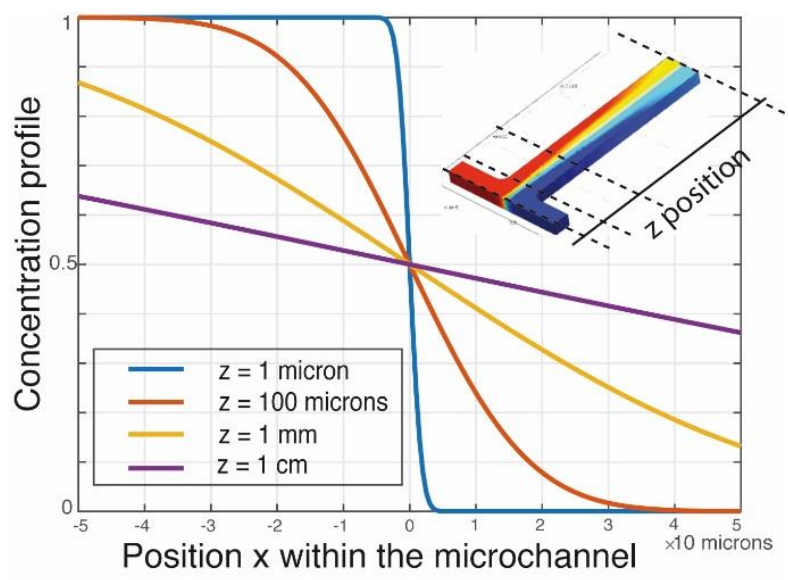

Fig. 2.7. Concentration profile evolution of a specie: time of diffusion is linked to convection travel in the channel

The formula (Eq. 2.25) can be used to estimate the passive mixing between two medium (case of the T-channel), to study diffusion of nanoparticles [23], for estimation of $D$, or concerning sorting molecules with there size in relation with their coefficient of diffusion (case of $\mathrm{H}$ filter) [24].

\subsubsection{Two phase flow: droplet generation within microfluidic devices}

The use of droplet within microfluidic chip permits the development of device with high numbers of unit system on which biological or chemical species can be isolated [25]. Each droplet becomes a microreactor with its own properties. It reduces drastically the contamination or cross-pollution during the experiments. To do so, basic operations such as generation, fusion, breakup or storage of the droplets have to be mastered [26]. The capillary phenomenon is used to fabricate and control droplet within a microchannel. Considering immiscible fluids, such as water and oil, the surface tension between the water/oil interface will drive the capability of generating droplet and keep it stable.

\subsubsection{Surface tension: basics}

The origin of the surface tension is due to the molecular force discontinuity, which appears at the interface between materials (Fig. 2.8). The interactions between the molecules in the interfacial zone induce a surface energy to keep the interface stable. It's expression $(\gamma$ or $\sigma)$ is in $[\mathrm{mN} / \mathrm{m}]$ or $\left[\mathrm{mJ} / \mathrm{m}^{2}\right]$ corresponding to an energy per unit surface $(\delta W)$ or a force per unit length $(\delta F)$. It corresponds to the energy needed to increase of $1 \mathrm{~m}^{2}$ the surface (Eq. 2.26).

As consequence, pressure discontinuity is the first effect of surface tension. In fact, surface tension corresponds to the pressure jump $\Delta \mathrm{P}$ that is induced between the two faces of an interface between two liquids in relation with its curvature. Its expression comes from Laplace's law applied to a droplet.

$$
\delta W=\sigma d S \text { or } \delta F=\sigma d l \text { and } \Delta P=P^{+}-P^{-}=\frac{2 \sigma}{R}
$$



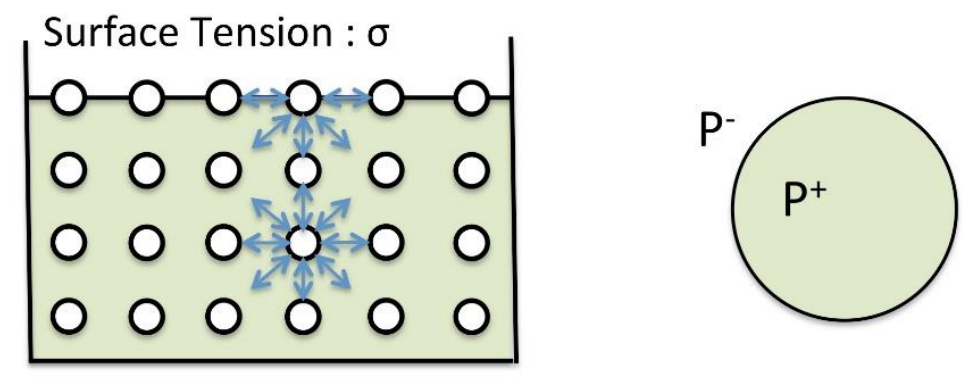

Fig.2.8. : Surface tension at the interface between two medium

\subsubsection{Droplet on a surface: wetting effect}

The curvature of the interface gives the pressure discontinuity. This effect occurs also with capillary phenomena when a triple interface is achieved, corresponding to a Solid/Liquid/Air interface. This is the case of a liquid droplet on a surface. At the equilibrium, from a local point of view, the droplet contact angle $\theta$ (Fig. 2.2.9) depends on the three surface tension parameters with the relation :

$$
\sigma_{S L}+\sigma_{L A} \cos \theta=\sigma_{S A}
$$
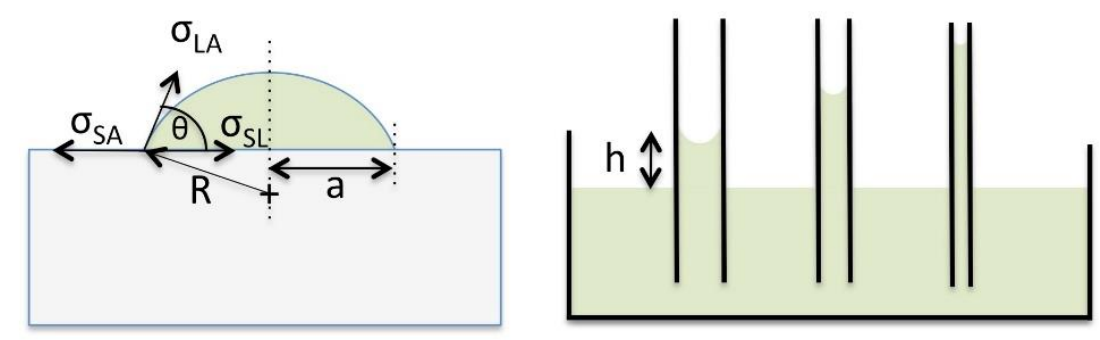

Fig. 2.9. : Capillary effect induces by surface tension

The pressure within the droplet can be calculated taking into account the interface curvature. The pressure drop is:

$$
\Delta P=\frac{2 \sigma_{L A}}{R} \text { with } R=\frac{a}{\cos \theta}
$$

The solid surface wetting property influences the curvature of the interface. Depending on contact angle value, the surface is Hydrophilic for $\theta<90^{\circ}$ and Hydrophobic for $\theta>90^{\circ}$

A well-known effect is the Jurin's law describing the rising (or falling) of a fluid column within a hydrophilic (or hydrophobic) tube (with a radius a). The capillary pressure at the interface induces an equivalent height ( $h$ ) of water column by hydrostatic equilibrium (Fig. 2.9). The height of liquid is given by:

$$
h=\frac{2 \sigma \cos \theta}{\rho g a}
$$

\subsubsection{Application in microfluidics: in-situ droplet generation in two phase flow}

Taking advantage of miniaturisation ( $\mathrm{Re}<<1$ : laminar flow, $\mathrm{Bo}<<1$ and $\mathrm{Ca}<<1$ : capillary dominates and stable interface), combining two immiscible fluids such as water and oil is a way to generate highly reproducible droplets within microchannel (Fig. 2.10). 
To modify the interface between the two fluids and generate a segmented flow composed of droplets, the shear stresses (viscous forces) must be able to break the surface tension (capillary forces) at the interface. It had been demonstrated by Thorsen et al. (2001) using a T Junction channel [27] (Fig. 2.10.B). A column of fluid (water) is pinched and breaks by another fluid (oil) coming perpendicularly. The flow focusing design (Fig. 2.10.A), based on the injection of the segmented phase coaxially into the continuous phase, had been proposed by Anna et. all in 2003 [28]. Droplets are emitted periodically at the nozzle of the flow focusing.

These two topologies are commonly used in droplet based microfluidic technology due to their simplicity of fabrication and use [29]. Highly monodisperse microdroplets are produced at $\mathrm{kHz}$ rates and generally made of aqueous solution dispersed in a continuous non-miscible inert carrier oil phase [15].
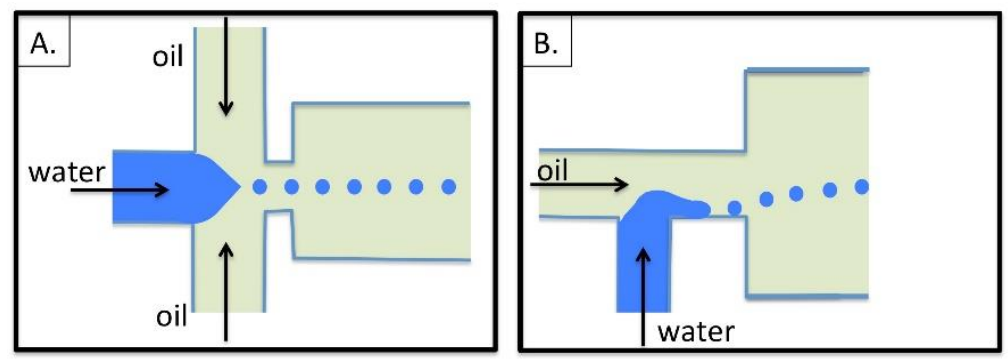

Fig. 2.10. Droplet generation by (A.) flow focusing or (B.) T-channel configuration

In order to stabilize the droplets and avoid coalescence between them, a surfactant is added to the continuous phase. Surfactants are molecules composed of two parts: one hydrophobic (tail) and one hydrophilic (polar head). It creates a layer at the interface that prevent coalescence between contacting droplets [5]. Of course, it affects the surface tension value by decreasing it.

In order to estimate the droplet radius formation, a balance between the surface tension and the viscous stresses can be calculated (Eq. 2.30). The first tends to reduce the interfacial area in order to minimize the energy, the second tries to extend the interface following the stream. At the limit of the droplet formation, the equilibrium can be express as the equality between the two stresses at the interface associated to the pressure effect:

$$
\Delta P=\frac{2 \sigma}{R} \leftrightarrow \tau_{w}=-\eta \frac{\delta v}{\delta y}=\eta \frac{2 v_{\max }}{h}
$$

We can extract an estimate droplet radius expression:

$$
R=\frac{\sigma}{\eta v_{\max }} h \cong \frac{h}{C_{a}}
$$

From that expression (Eq. 2.31), the capillary number is introduced to show the dependence of the droplet radius with $\mathrm{C}_{\mathrm{a}}$ which is in relation with the balance between interfacial pressure $(\Delta P)$ and viscous stress $\tau_{w}$.

\subsubsection{Centrifugal device: Lab On Disc}

Compare to pressure driven microfluidic, where the flow is induced by differential pressure or by using syringe pump, spinning device used the centrifugal forces to drive liquids within microchannel (Fig. 2.11). Usually named as Lab On Disc microfluidic [30], it's similar to a Compact Disc with microchannel array embedded within its material. 


\subsubsection{Lab On Disc principle}

Inertial forces are exploited to induce the liquid displacement [31]. Considering a punctual mass (M) within the LOD submitted to the angular velocity $\Omega$, three forces act on $M$ :

- Centrifugal force : $\vec{F}_{c e n t}=M \cdot r \Omega^{2} \overrightarrow{u_{r}}$, which is a radial force similar to the gravity $\left(g_{c} \sim r . \Omega^{2}\right)$ acting on the mass, oriented towards the external of the LOD.

- Coriolis force : $\vec{F}_{c o r}=2 M \frac{\overrightarrow{d r}}{d t} \otimes \vec{\Omega}$, oriented perpendicular to both the angular velocity and liquid velocity vector.

- Euler force : $\vec{F}_{E}=M r \cdot \overrightarrow{u_{r}} \otimes \frac{\overrightarrow{d \Omega}}{d t}$

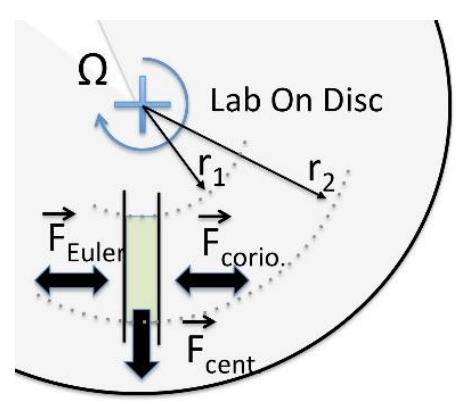

Fig. 2.11. Use of a Compact Disc format for centrifugal driven flow

These three forces are handled in order to manipulate liquid within microchannel. In the context of microfluidics, the force density ( $f$ ) is used and associated to the negative gradient of pressure with the relation: $\vec{f}=-\overrightarrow{g r a d} P$.

\subsubsection{Centrifugal flow within microfluidic channel}

Considering a rotating channel filled with a liquid column from distance $\mathrm{r} 1$ and $\mathrm{r} 2$ to the center, the pressure density induces by the centrifugal force can be written as :

$$
\rho r \Omega^{2}=-\frac{\delta P}{\delta r}
$$

The pumping pressure generated is thus :

$$
\Delta P_{2-1}=-\frac{\rho \Omega^{2}}{2}\left(r_{2}^{2}-r_{1}^{2}\right)
$$

This differential pressure (Eq. 2.32), defined as the centrifugal pressure applied on the liquid, drives the liquid radially outward and can be associated to a laminar flow (microfluidic context) as studied in previous section. Supposing a circular channel (diameter $D_{H}$ ), the mean velocity expression obtained is:

$$
V=\frac{\rho D_{H}^{2} \Omega^{2}}{32 \eta}\left(\frac{r_{1}+r_{2}}{2}\right)
$$

Based on a micro-channel with a diameter of $100 \mu \mathrm{m}$, with a filling of water from $r_{1}=2 \mathrm{~cm}, r_{2}=4 \mathrm{~cm}$ and a spinning of $10 \mathrm{round} / \mathrm{sec}$, the centrifugal effect gives:

$$
\Delta P_{1-2}=2.4 \mathrm{kPa}-\mathrm{V}=3.7 \mathrm{~cm} . \mathrm{s}^{-1}
$$




\subsubsection{Valve in the flow: capillary effect}

In order to control the fluid injection, the use of capillary effect or local hydrophobic surface enables to stop the flow under a limited pressure (Fig. 2.12), which above the flow can pass and continue [32].

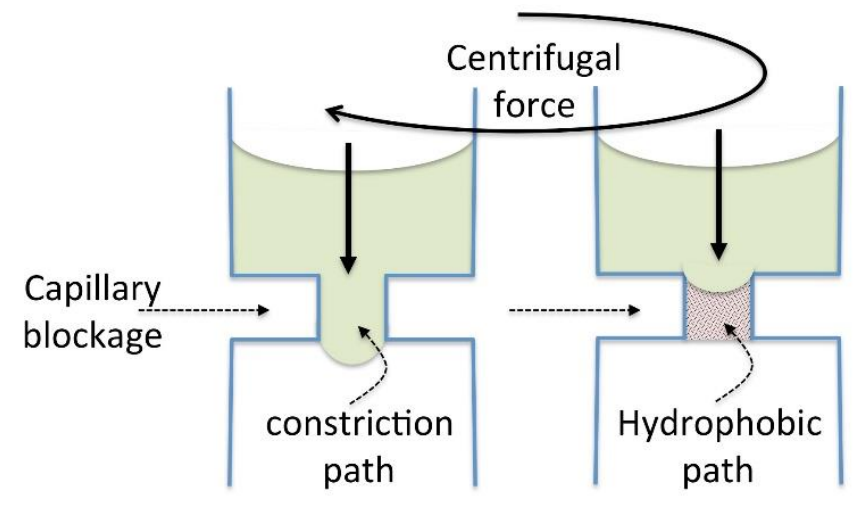

Fig. 2.12. : Capillary effects to control the flow path with centrifugal force

Using previous demonstration, supposing a circular channel, the valve blocks the liquid until (Eq. 2.35):

$$
\Delta P_{2-1}=-\frac{\rho \Omega^{2}}{2}\left(r_{2}^{2}-r_{1}^{2}\right) \leftrightarrow \Delta P_{\sigma}=\frac{4 \sigma \cos \theta}{d}
$$

It give a burst angular velocity above which the liquid can pass (Eq. 2.36):

$$
\Omega_{b}=\sqrt{\frac{8 \sigma \cos \theta}{d \rho\left(r_{2}^{2}-r_{1}^{2}\right)}}
$$

This principle is used for example when a quantified liquid need to be delivered (Fig. 2.13). Controlled volume [33] is stored in a chamber and is delivered by using an angular velocity above $\Omega_{b}$.

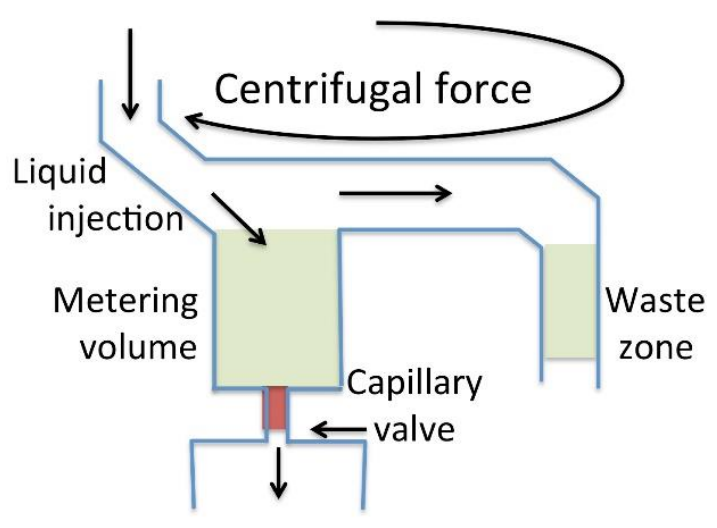

Fig. 2.13. Use a chamber for metering sample volume

In microfluidic, due to the rectangular section of the channel, the principle is still the same but the relation is a little more complex in order to take into account the cross section of the channel. For mixing application, Eulor force is used by angular velocity oscillation with time $\left(\frac{d \Omega}{d t}\right)$. The rapid change in spinning velocity permits advective flow that induces the mixing [4]. 


\subsubsection{Paper based microfluidics: lateral flow}

\subsubsection{Capillary driven flow within paper}

In 2005, Pr Whitesides had proposed the principle of developing microfluidic within paper [34]. Aim is to get low cost medical devices for point of care diagnostic. The idea is to use the capillary effects to induce diffusion of liquid inside paper and to shape the flow with "wax" integration. The paper is composed of pressed cellulosic fibres which behave like a hydrophylic porous material [35]. Here, the diffusion is guided by hydrophobic wall integrated within the paper by printing technic associated to thermal inclusion (Fig. 2.14) [6]. By this principle the fluid can be driven towards specific reaction zone in order to achieve chemical analysis.

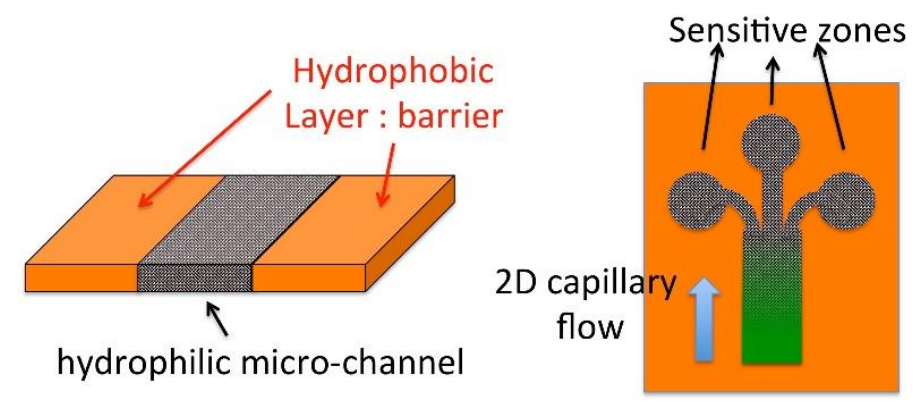

Fig. 2.14. Paper based microfluidic : lateral flow using capillary effect within paper drives the liquid

\subsubsection{Simple model associated: Lucas-Washburn law}

The Lucas-Washburn law describes at a first approximation the fluid displacement within a paper. It gives the distance $L$ traveled be the fluid front for a time $t$ with $R_{p}$ the average pore radius (Fig. 2.15) [36]. In this description, evaporation and gravity effects are not taking into account. The driving force is the capillary effects with the surface tension $\sigma$, the viscous force defines the speed rate with equivalent hydraulic resistance $\left(R_{H p}\right)$ of the pore and the flow rate $(\mathrm{Q})$.

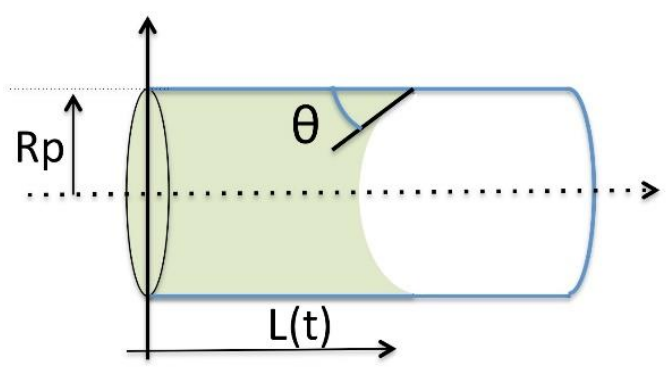

Fig. 2.15. Fluid front representation in a circular channel

Supposing an equivalent channel of radius $R_{p}$, filled of liquid on a distance $L$, the flow rate generated by the capillary forces is:

$$
\Delta P_{c}=\frac{2 \sigma \cos \theta}{R_{p}}=R_{H p} * Q=\left(\frac{8}{\pi} \eta \frac{L}{R_{p}^{4}}\right) *\left(\pi R_{p}^{2} \frac{d L}{d t}\right)
$$

The evolution of the fluid front to a distance $L$ is thus: 


$$
L \frac{d L}{d t}=\frac{\sigma \cos \theta R_{p}}{4 \eta} \Rightarrow L(t)=R_{p} \sqrt{\frac{t}{\tau_{a d v}}} \text { with } \tau_{a d v}=\frac{2 \eta R_{p}}{\sigma \cos \theta}
$$

The dynamics of the fluid front is square root dependence with time (Eq. 2.38). The characteristic time of liquid advection within the paper depends on the porous radius and viscosity to surface tension ratio. This average 1D model for fluid transport with scaffold of cellulose is a first approach to control the interaction time between reagents in an assay (Eq. 2.36). More complex models need to include shape of the channel, the evaporation rate and gravity effect.

\subsection{Biomolecular interactions (Morgan Madec)}

This chapter aims at giving the reader a background in biochemistry required to understand the structure of the main chemical compounds of living matter (carbohydrates, proteins, DNA ...), their role in biological functions as well as their interactions. The following questions are discussed: What a protein is made of? How does DNA give a protein? How in turn proteins act on living matter to perform biological functions? What is the role of other molecules such as carbohydrates or lipids? What is an antibody? An enzyme? How do they all interact? Finally, the last part of this chapter deals with mathematical modeling associated with those biological mechanisms.

\subsubsection{From atoms to proteins}

This section condenses the main principles of biochemistry. Only the concepts required for the understanding of biological mechanisms described along this book are discussed. Some of them have been simplified in order to the facilitate reader's understanding. For a deeper dive, we suggest the book Biochemistry written by J.M. Berg, J.L. Tymoozko and G.J. Gatto [37].

\subsubsection{Atoms and molecules}

Mendeleyev periodic table of chemical elements is composed of 118 entries. In this chapter, focus is put on six of them which are the most abundant in living matter. There are often designated by the acronym CHONPS for carbon $(\mathrm{C})$, hydrogen $(\mathrm{H})$, oxygen $(\mathrm{O})$, nitrogen $(\mathrm{N})$, phosphorus $(\mathrm{P})$ and sulfur $(\mathrm{S})$. They are summarized in Table 2.2. The residual is composed of ions and other atoms, the most relevant being the calcium which is as abundant as sulfur in some organisms.

Except for some elements, namely the noble gases, a standalone atom is not stable because of an incomplete external electron shell. To reach stability, they share electrons with other atoms in order to form molecules. A shared pair of electrons is a covalent bond and the number of covalent bond an atom should have to be stable corresponds to its valence (Table 2.2). The simplest example is the dihydrogen $\left(\mathrm{H}_{2}\right)$ molecule, composed with two $\mathrm{H}$ atoms sharing one covalent bond (Figure 2.16A).

Electron sharing is not always equitable because some atoms can be more avid of electron than others. In this case, the electron pair is pulled by the most avid atom and becomes a polarized molecule. The "pulling power" of atoms is quantified by their electronegativity (Table 2.2). In the example of dihydrogen, both atoms have the same electronegativity and the covalent bond is equitably shared between atoms. The molecule is nonpolar. In the case of water $\left(\mathrm{H}_{2} \mathrm{O}\right.$, Figure 2.16B), as the electronegativity of oxygen is much higher than that of hydrogen atom, the two electron pairs are pulled by the oxygen atoms, and the molecule becomes polar. It is commonly admitted that a molecule is considered to be polar as soon as the electronegativity gap between the atoms involved in covalent bonds is over 0.4. As a consequence, H-N, C$\mathrm{O}$ and $\mathrm{C}-\mathrm{N}$ bonds leads to polarized molecules whereas $\mathrm{H}-\mathrm{C}$ and all bonds with two similar atoms are considered as nonpolar. 
Moreover, the arrangement of atoms in a molecule always corresponds to the configuration that minimizes its free energy induced by electrostatic forces at the microscopic level. For instance, a water molecule is $\mathrm{V}$ shaped and both $0.95 \AA \AA$-long $\mathrm{O}-\mathrm{H}$ bonds form a $104.45^{\circ}$ angle. For small molecules, these configurations can be deduced from theoretical consideration. For larger molecule, computer software such as Avogadro [38] or Molview [39] should be used.

\begin{tabular}{|c|c|c|c|c|c|c|}
\hline & Symbol & $\begin{array}{c}\text { Atomic } \\
\text { number }\end{array}$ & Valence & $\begin{array}{c}\text { Atomic } \\
\text { mass }\end{array}$ & $\begin{array}{c}\text { Electro- } \\
\text { negativity }\end{array}$ & $\begin{array}{c}\text { Abundance } \\
\text { rate }\end{array}$ \\
\hline Hydrogen & $\mathrm{H}$ & 1 & 1 & 1.008 & 2.30 & $63 \%$ \\
\hline Oxygen & $\mathrm{O}$ & 8 & 2 & 15.999 & 3.44 & $24 \%$ \\
\hline Carbon & $\mathrm{C}$ & 6 & 4 & 12.001 & 2.55 & $10 \%$ \\
\hline Nitrogen & $\mathrm{N}$ & 7 & 3 & 14.007 & 3.04 & $1.4 \%$ \\
\hline Phosphorus & $\mathrm{P}$ & 15 & 3 or 5 & 30.973 & 2.19 & $0.2 \%$ \\
\hline Sulfur & $\mathrm{S}$ & 16 & 2,4 or 6 & 32.060 & 2.58 & $0.1 \%$ \\
\hline
\end{tabular}

Table 2.2 - List of the most common atoms in living matter with associated chemical properties
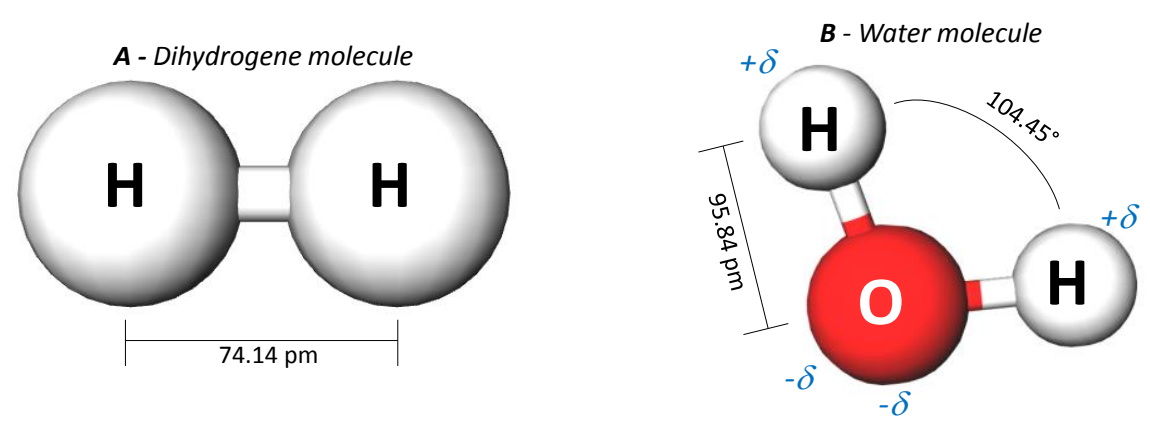

Fig.2.16 - Representation of Dihydrogen and water molecules

\subsubsection{Functional groups}

There are plenty of molecules in living matter corresponding to different combinations of atoms. To analyze and predict the properties of a given molecule, it is often easier to split the molecule into functional groups. There are dozens of functional groups. A non-exhaustive list of those most commonly met in living matter is given in the following (Figure 2.17).

i. Methyl group is only composed of a $\mathrm{C}-\mathrm{H}$ bond (the number of $\mathrm{H}$ being adjusted to meet the valence of C). Methyl groups can be connected together to form a carbon chain with single, double or triple $\mathrm{C}-\mathrm{C}$ covalent bonds. Molecules made of methyl groups are hydrocarbons.

ii. Phenyl group is a 6-C cycle with alternating single and double $\mathrm{C}-\mathrm{C}$ bonds. Molecules constituted of such a group are benzene derivatives.

iii. Hydroxyl group is an $\mathrm{O}-\mathrm{H}$ bond that replaces an $\mathrm{H}$ in a carbon chain. A carbon chain with at least one hydroxyl group is an alcohol (e.g. ethanol). The $\mathrm{O}-\mathrm{H}$ bond renders a molecule polarized.

iv. Amino group is composed with $\mathrm{N}-\mathrm{H}$ bond. An amino group can be inserted in a carbon chain, forming an amine (e.g. methylamine).

v. Aldehyde group is a methyl group in which a double $\mathrm{C}=\mathrm{O}$ bond replaces a $\mathrm{C}-\mathrm{H}$ bond. The carbon chain with at least one aldehyde group is an aldehyde (e.g. formaldehyde). $\mathrm{C}=\mathrm{O}$ bond make them polarized.

vi. Carboxyl group combines on the same carbon an aldehyde and a hydroxyl group. Carboxyl groups can tail a carbon chain to form a carboxylic acid (e.g. acetic acid), which is also a polarized molecule.

vii. Phosphate group is composed of a phosphorus atom with two $\mathrm{P}-\mathrm{OH}$ bonds, single $(\mathrm{P}-\mathrm{O})$ and a double $(\mathrm{P}=\mathrm{O})$ bond. One of the oxygen atom of the $\mathrm{P}-\mathrm{O}$ bond can be connected to carbon chains, 
other phosphate groups or more complex molecules. Phosphate groups can also lose one or several $\mathrm{H}$ of $\mathrm{P}-\mathrm{OH}$ bonds to become phosphate ions.

viii. The sulfhydryl group is an equivalent of the hydroxyl group where sulfur replaces oxygen.
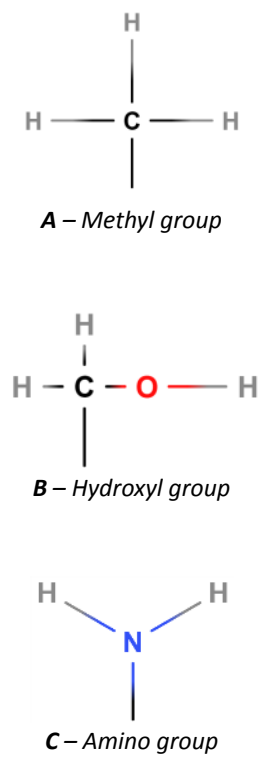
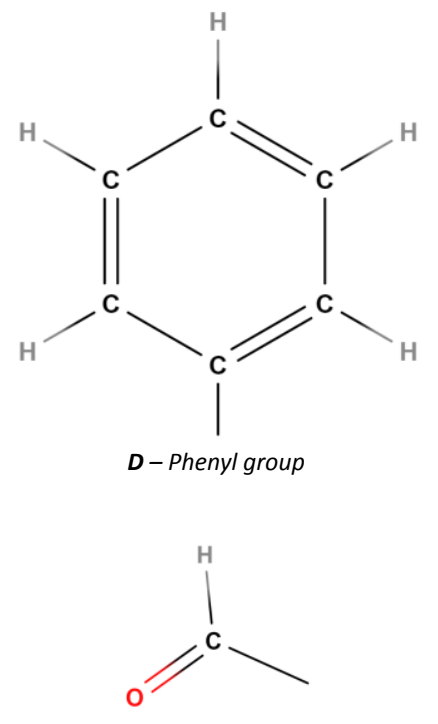

E-Aldehyde group
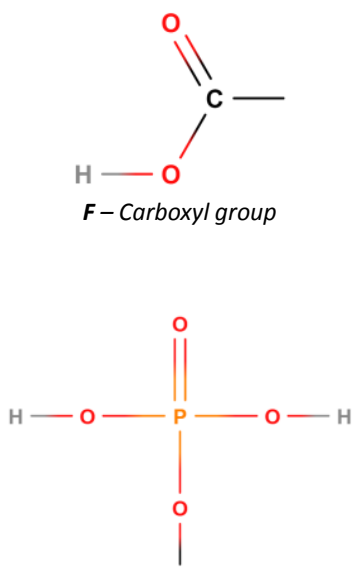

G-Phosphate group

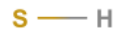

$\boldsymbol{H}$-Sulfhydryl group

Fig. 2.17 - Representation of the most common functional groups in living matter

\subsubsection{Weak bonds}

Beside covalent bonds, other weaker interaction may occur between molecules or between atoms from the same molecule. These interactions are classified into four main categories:

i. Ionic bonds are the most straightforward. Ions are charged molecules for which one or several atoms have a number of covalent bond that are either above or below the expected one (valence). When a negatively charged ion meets a positively charged ion, they attract each other and form an ionic bond. For example, positive sodium ion bind to negative chloride ion in order to form $\mathrm{NaCl}$ (salt).

ii. Hydrogen bonds rely on the same principle except that they involve polar molecules instead of ions: the positive pole of a polar molecule attracts the negative pole of other molecules. The term "hydrogen bond" comes from the fact that because of its weak electronegativity, hydrogen is always the positive pole of a polar molecule. Hydrogen bonds occur for instance between two water molecules: one of the hydrogen atoms of a molecule attracts the oxygen atom of another one. These bonds are responsible for the crystalline structure of liquid water and ice.

iii. Hydrophobic bonds occur between hydrophobic molecules. As water is polar, nonpolar molecules cannot bind to water. As a consequence, nonpolar molecules are called hydrophobic. If several hydrophobic molecules are put in contact with water, they tend to clump up together by forming hydrophobic bonds in order to reduce their contact surface with water. Hydrophobic bonds occur for instance between oil molecules in water and lead to emulsions.

iv. Van der Walls bonds are induced by Van der Walls forces at atomic level and may happened between atoms or molecules, even if there are nonpolar.

\subsubsection{Four main classes of organic macromolecules}

In this fourth subsection, the four most common classes of macromolecules encountered in living matter are described.

\section{Carbohydrates}


Carbohydrates are macromolecules composed of carbon, hydrogen and oxygen. There are also called sugars and the most common one is the glucose $\left(\mathrm{C}_{6} \mathrm{H}_{12} \mathrm{O}_{6}\right)$. In living matter, glucose (as well as many other carbohydrates) is involved in a complex set of reactions (glycolysis metabolic pathway) in order to be converted into other carbohydrates, amino acids (see below), water and carbon dioxide. Glucose is also considered to be a source of energy for life as the glycolysis release a large amount of energy.

\section{Lipids}

Lipids are often assimilated to fats. Most of them are long hydrocarbon chains headed or tailed by specific functional group. The long hydrocarbon chain makes them hydrophobic or at the least amphiphilic (composed of a hydrophilic head and a long hydrophobic tail). Lipids have two main function in living matter.

Firstly as for the glucose, the degradation of lipids (especially the triglycerides) by specific metabolic pathways releases a great amount of energy. Secondly, lipids can make hydrophobic bonds with one other in order to form long damp-proof macrostructures called micelle, liposome or bilayer sheets. For instance the cell membrane consists of a bilayer of amphiphilic phospholipids whose hydrophobic tails are bond together and hydrophilic heads exposed to the inside and outside of the cell (cf. section 2.17).

\section{Amino acids and proteins}

An amino acid is a molecule composed of a core with two carbons, one with a carboxyl group and the other with an amino group and a side chain (radical) which differs from an amino acids to another (Figure 2.18A and 2.18B). There are only 20 different amino acids. They are listed in Table 2.3.

\begin{tabular}{|c|c|c|c|c|}
\hline Name & $\begin{array}{l}\text { 3-letter } \\
\text { symbol }\end{array}$ & $\begin{array}{l}\text { 1-letter } \\
\text { symbol }\end{array}$ & Side Chain formula & Property \\
\hline Alanine & Ala & A & $-\mathrm{CH}_{3}$ & Hydrophobic \\
\hline Arginine & Arg & $\mathrm{R}$ & $-\left(\mathrm{CH}_{2}\right)_{3}-\mathrm{NH}-\mathrm{CNH}_{2}(\mathrm{NH})$ & Basic (pos. ion) \\
\hline Asparagine & Asn & $\mathrm{N}$ & $-\mathrm{CH}_{2}-\mathrm{CONH}_{2}$ & Polar uncharged \\
\hline Aspartic Acid & Asp & D & $-\mathrm{CH}_{2}-\mathrm{COOH}$ & Acidic (neg. ion) \\
\hline Cysteine & Cys & C & $-\mathrm{CH}_{2}-\mathrm{SH}$ & Disulfide bridges \\
\hline Glutamic Acid & Glu & $\mathrm{E}$ & $-\left(\mathrm{CH}_{2}\right)_{2}-\mathrm{COOH}$ & Acidic (neg. ion) \\
\hline Glutamine & Gln & Q & $-\left(\mathrm{CH}_{2}\right)_{2}-\mathrm{CONH}_{2}$ & Polar uncharged \\
\hline Glycine & Gly & G & $-\mathrm{H}$ & Structural flexibility \\
\hline Histidine & His & $\mathrm{H}$ & $-\mathrm{CH}_{2}-\mathrm{C}_{3} \mathrm{H}_{2} \mathrm{NNH}$ & Basic (pos. ion) \\
\hline Isoleucine & Ile & I & $-\mathrm{CH}-\mathrm{C}_{2} \mathrm{H}_{5}\left(\mathrm{CH}_{3}\right)$ & Hydrophobic \\
\hline Leucine & Leu & L & $-\mathrm{CH}_{2}-\mathrm{CH}-\mathrm{CH}_{3}\left(\mathrm{CH}_{3}\right)$ & Hydrophobic \\
\hline Lysine & Lys & $\mathrm{K}$ & $-\left(\mathrm{C}_{2} \mathrm{H}_{4}\right)_{4}-\mathrm{NH}_{2}$ & Basic (pos. ion) \\
\hline Methionine & Met & $M$ & $\left(\mathrm{CH}_{2}\right)_{2}-\mathrm{S}-\mathrm{CH}_{3}$ & Hydrophobic \\
\hline Phenylalanine & Phe & $\mathrm{F}$ & $-\mathrm{CH}_{2}-\mathrm{C}_{6} \mathrm{H}_{5}$ & Hydrophobic \\
\hline Proline & Pro & $P$ & $-\mathrm{C}_{3} \mathrm{H}_{6}$ looped & Structural rigidity \\
\hline Serine & Ser & $\mathrm{S}$ & $-\mathrm{CH}_{2} \mathrm{OH}$ & Polar uncharged \\
\hline Threonine & Thr & T & $-\mathrm{CH}(\mathrm{OH})-\mathrm{CH}_{3}$ & Polar uncharged \\
\hline
\end{tabular}




$\begin{array}{lllll}\text { Tyrosine } & \text { Tyr } & \mathrm{T} & -\mathrm{CH}_{2}-\mathrm{C}_{6} \mathrm{H}_{4} \mathrm{OH} & \text { Hydrophobic } \\ \text { Tryptophan } & \text { Trp } & \text { W } & \mathrm{CH}_{2}-\mathrm{C}_{4} \mathrm{NH}-\mathrm{C}_{4} \mathrm{H}_{4} & \text { Hydrophobic } \\ \text { Valine } & \text { Val } & \mathrm{V} & -\mathrm{CH}^{-} \mathrm{CH}_{3}\left(\mathrm{CH}_{3}\right) & \text { Hydrophobic }\end{array}$

Tab. 2.3 - List of the 20 amino acids

Amino acids form peptide bonds together to create long polypeptidic chains called proteins. A peptide bond is produced by the reaction between the $\mathrm{N}$ of the amine group of a given amino acid and the $\mathrm{C}$ of the carboxyl group of the other one. Thus, it releases a water molecule (Figure 2.18C). The number of amino acids in a protein chain may vary from ten to thousands. Due to the electrical properties of each amino acid, the shape of the chain is very complex and can be declined at four levels:

i. The primary structure corresponds to the sequence of amino acids.

ii. The secondary structure describes the way the side chains are organized. The organization is mainly driven by hydrogen bonds occurring between successive side chains. The two most common structures are the alpha-helix and the beta-sheet. The secondary structure is mainly influenced by the position of glycines and prolines in the chain.

iii. The tertiary structure is the way the protein is folded at the molecule scale. Folding is driven by a set of weak bonds described in 2.3.1.3. Protein folding can be predicted with the help of complex computing algorithms such as PyMOL [40].

iv. A protein is sometimes an assembly of several polypeptide chains called sub-units. The quaternary structure describe this assembly. Hemoglobin, a protein used to fix and transport oxygen in blood, is an example of tetramer composed of two couples of sub-units, the $\alpha$-chain and the $\beta$-chains.
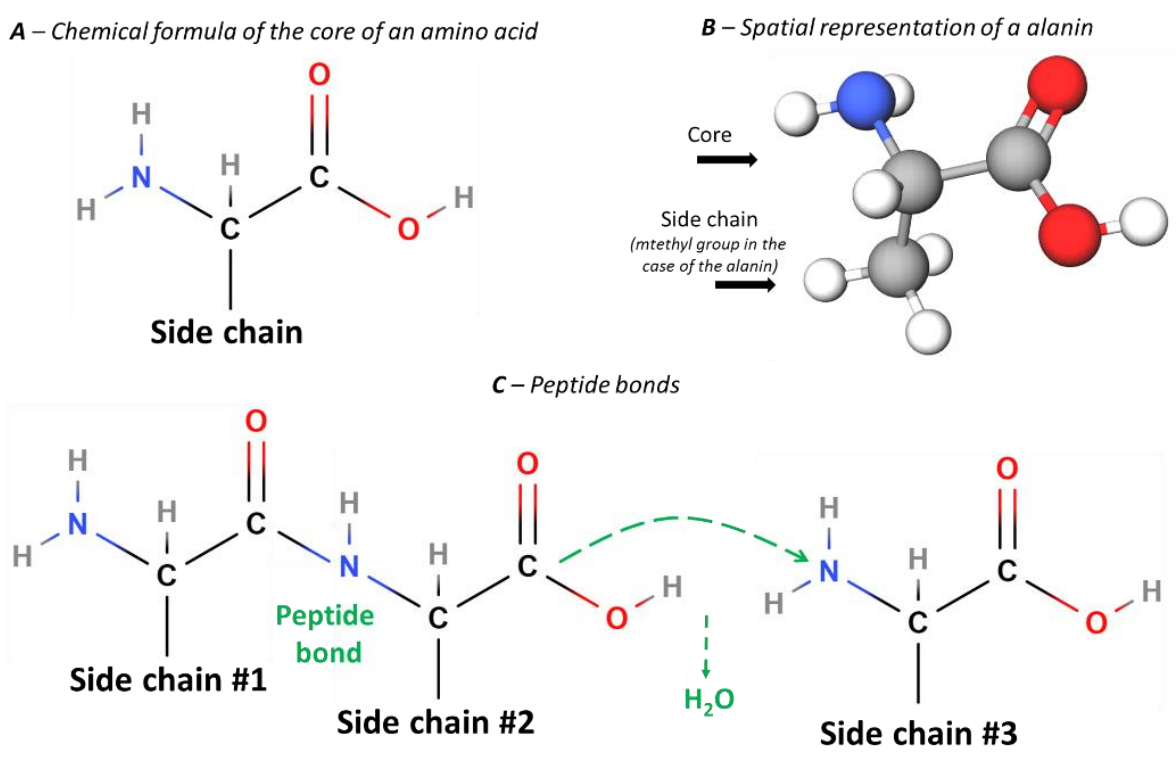

Fig. 2.18 General formula of an amino acid (A), 3D representation of the alanine (B) and the peptide bond reaction between successive amino acids to form protein (C)

\section{Nucleotides and nucleic acids}

A nucleotide is molecule composed of a five-carbon sugar, up to three phosphate groups and a nitrogenous base. Nucleotides are the building blocks of nucleic acids. The most famous nucleic acid is probably the deoxyribonucleic acid (DNA), which encodes the genome that can be considered as the blueprint of living matter. In this case, the five-carbon sugar is a deoxyribose 5-phosphate and the nitrogenous base is an adenine $(\mathrm{A})$, a thymine $(\mathrm{T})$, a guanine $(\mathrm{G})$ or a cytosine $(\mathrm{C})$ (Figure 2.19A). 
The sugar of a nucleotide can bind to the phosphate group of the next nucleotide in the chain (phosphodiester bond) in order to form a DNA strand (Figure 2.19B). The structure of DNA is a double-helix composed of two complementary DNA strands, as predicted by Watson and Crick in 1953. In this structure, $A$ always faces $T$ and $C$ always faces $G$. This complementarity is due to the fact that $A$ and $T$ exhibit 2 potential hydrogen bonds while $G$ and $C$ exhibit three (Figure 2.19C-F). To identify the carbon is the deoxyribose, they are numbered from $1^{\prime}$ to $5^{\prime}$, as depicted in Figure 2.19A. This notation is also used to identify the first ( $\left.5^{\prime}\right)$ and the last $\left(3^{\prime}\right)$ nucleotide of a DNA chain.

Binding and separation of DNA strands are respectively called hybridization and denaturation. The ratio of hybridized form over denatured form of DNA molecule is an empirical low which mostly depends on the temperature $T$, the length of DNA strand $N$, the ratio $\eta$ of G-C pairs (as the triple hydrogen bond of G-C is stronger than the double for $A-T$ ) and the concentration of positive ions such as calcium [ $\left.\mathrm{Na}^{+}\right]$(which operate as a screen preventing the two DNA strands to repulse each other due to negatively charges backbone). The temperature at which $50 \%$ of the DNA strands are hybridized is given by [41]:

$$
T_{50 \%}=81.5+16.6 \cdot \log _{10}\left(\left[\mathrm{Na}^{+}\right]\right)-\frac{600}{N}+0.41 \cdot \eta
$$
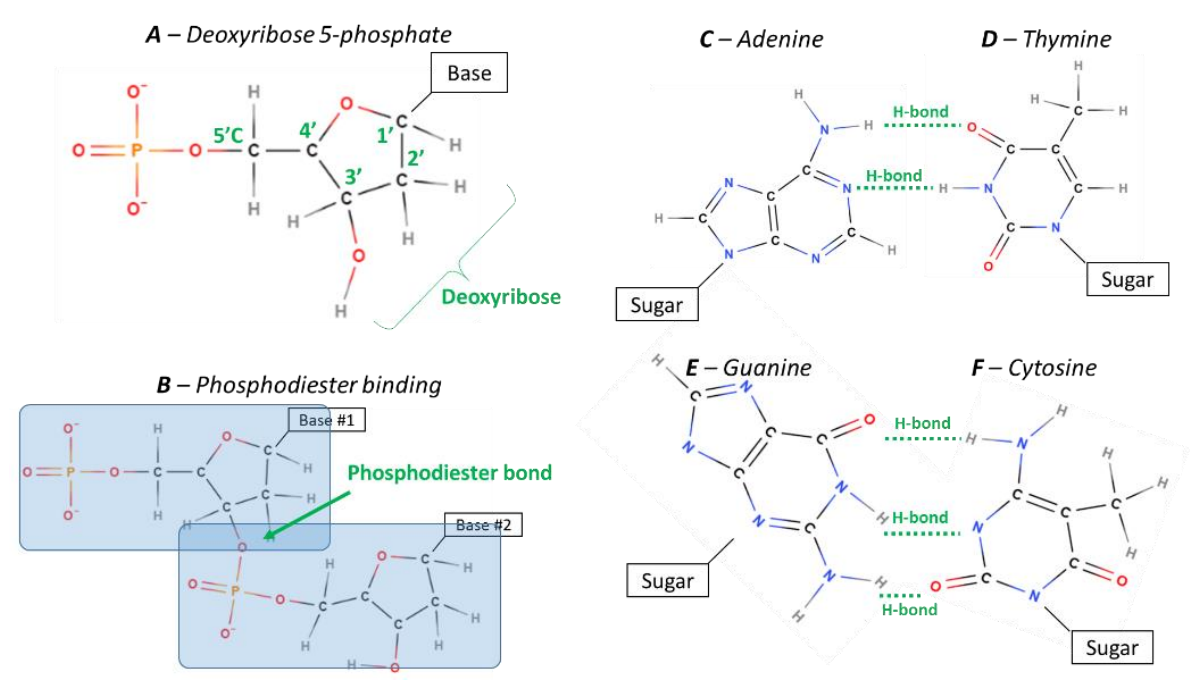

Fig. 2.19 Representation of a Deoxyribonucleotide (A), a phosphodiester bond between two deoxyribonucleotide (B) and the four nitrogenous bases of DNA ( $C$ to F). Hydrogen bonds between complementary bases are also represented in a green dotted line.

The second most common nucleic acid is the ribonucleic acid (RNA) composed of a ribose (which resembles a deoxyribose except that a $\mathrm{OH}$ replace the $\mathrm{H}$ on carbon 2', see Figure 2.3.4A), a phosphate group and a nitrogenous base (same as for DNA except that the uracil replaces the thymine). Relationships between DNA and RNA from genetic point of view will be described in the next subsection.

The last couple of nucleotides that deserve to be discussed are the adenosine triphosphate (ATP) and the adenosine diphosphate (ADP). For ATP, the sugar is a ribose, the nitrogenous base is an adenine and it is tailed by three phosphate groups (Fig. 2.20). Structures of ADP and ATP are the same except that ADP has only two phosphate groups. ATP's internal energy is very high due to the proximity of polarized P-O covalent bonds. ATP to ADP hydrolysis releases a large amount of energy which is often used to force reaction to occur (see Section 2.3.2.4). Conversely, ADP to ATP phosphorylation may also be coupled to reactions with very favorable energy balances in order to harvest released energy. In other words, the role of ATD and ADP in metabolism can be compared to the role of a battery in a hybrid vehicle (with the fuel and thermal motor corresponding respectively to glucose and glycolysis). ATP and ADP are used in every 
metabolic pathway: it is estimated that the mean mass of ATP in human body is about $250 \mathrm{~g}$ but total mass of ATP consumed and produced is about $50 \mathrm{~kg}$ a day.

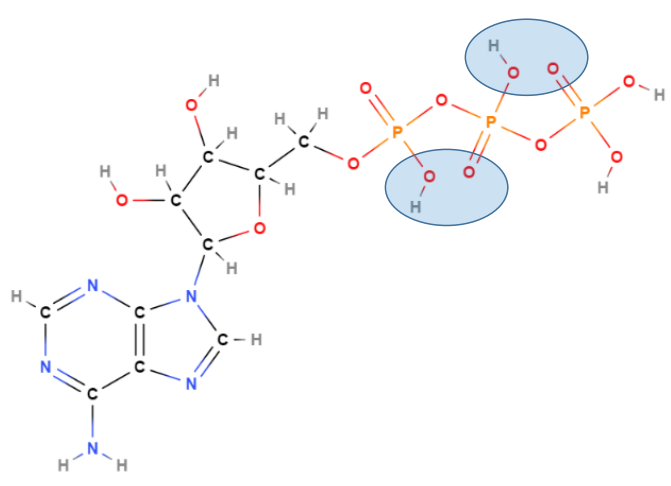

A - ATP Chemical Formula

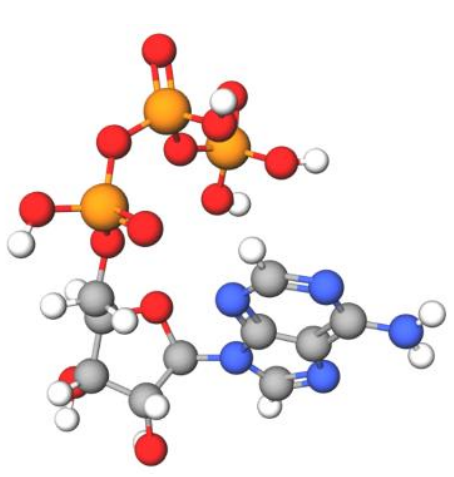

B - ATP 3D structure

Fig. 2.20 Adenosine Triphosphate Chemical formula and 3D representation

\subsubsection{From DNA to proteins}

The last part of this section complete the loop by describing how DNA and proteins are related.

\subsubsection{A brief history of genetic}

Genetic was born in the middle of the $19^{\text {th }}$ century and is still an important topic of investigation topic [42]. Making a long story short, modern genetics started in 1863 with George Mendel who studied seven characteristics of peas. He pointed out that something (a gene) in living matter should be responsible of the inheritance of several characteristics from a generation to another. Moreover, from statistical analysis, he also made the assumption that each individual has two genes, one from every parent, and that a dominance relationship exists between the different versions of these genes. It was confirmed later by Thomas Morgan's experiments on the fruit fly. Morgan introduced two new notions: the fact that some characteristics depend of the sex of the individual and the fact that several gene are grouped in chromosomes.

From 1928 to 1952, many experiment (Frederick Griffith, Oswald Avery and Alfred Hershey) have been carried out in order to find the nature of the "something" that transmit characteristics from an individual to another. They conduct experiments with mice and mice killer bacteria. They first pointed out that the "killing" characteristics can be acquired by a harmless bacteria in contact with molecules extracted from killer bacteria. With this in mind, they isolated all these molecules and reintroduced them one by one in a harmless bacterium. They observed that the bacteria become a killer after introduction of DNA.

The last part of the mystery was to understand how DNA created the biological function. The notion of mRNA, tRNA, codon, genetic code ... which will be described in the next session have been established during the 60 s by several scientists such as Ochoa, Khorana, Holley and Nirenberg. Since the 60s, advances in biotechnology and computer science led to major breakthrough in the understanding of genetic mechanisms, the deciphering of the genetic code, the study of relationships between genes and some diseases, the actions of several proteins on metabolism and even the complete decoding of the about 25,000 genes in the human body (Human Genome Project kicked off in 1990 and achieved in 2003).

\subsubsection{Transcription and translation of DNA}

The conversion from DNA to an active protein is a two (sometimes three) stage process. Firstly, one of the two DNA strands (namely the coding strand) is transcribed into a messenger RNA (mRNA) strand. Cells make a copies of the blueprint before manufacturing it instead of using its single original blueprint. The 
major actor of transcription is a specific protein, the mRNA polymerase, which reads one of the strand of DNA molecule (previously separated from the other strand by another molecule) and permits mRNA nucleotides to assemble together in front of the DNA coding strand. Of course, a cell does not transcript all its genes continuously. Most of the time, transcription is triggered by specific events and the transcription rate is regulated by the concentration of other molecules, the so-called transcription factors.

Secondly, mRNA is translated into an assembly of amino acid. Basically, three consecutive nucleotides in the mRNA sequence form a codon and each codon encodes for a given amino acid according to the genetic code (Fig. 2.21). With $64\left(4^{3}\right)$ combinations for 20 amino acids, the genetic code is redundant. Translation consists in reading the sequence of mRNA three by three and assembling the corresponding amino acid. This operation requires a ribosome and transfer RNAs (tRNA). tRNAs are molecules that exhibit at one side an amino acid and at the other side an anticodon (i.e. the sequence of three nucleotides which are complementary to the codon which codes for this amino acid). As a consequence, tRNA's anticodon recognizes its cognate codon on mRNA strand and bind to it. Ribosomes are complex molecules made of dozens of proteins. The ribosome is composed of two subunits: the smallest is in charge of codon recognition on $\mathrm{mRNA}$ and the biggest one is in charge of making peptide bonds between the amino acids of each tRNA. The ribosome binds at the beginning of mRNA strand and slides along it up to the stop codon.

The third stage, which is optional, consist in adding post-translational modifications on the translated proteins to make them functional.

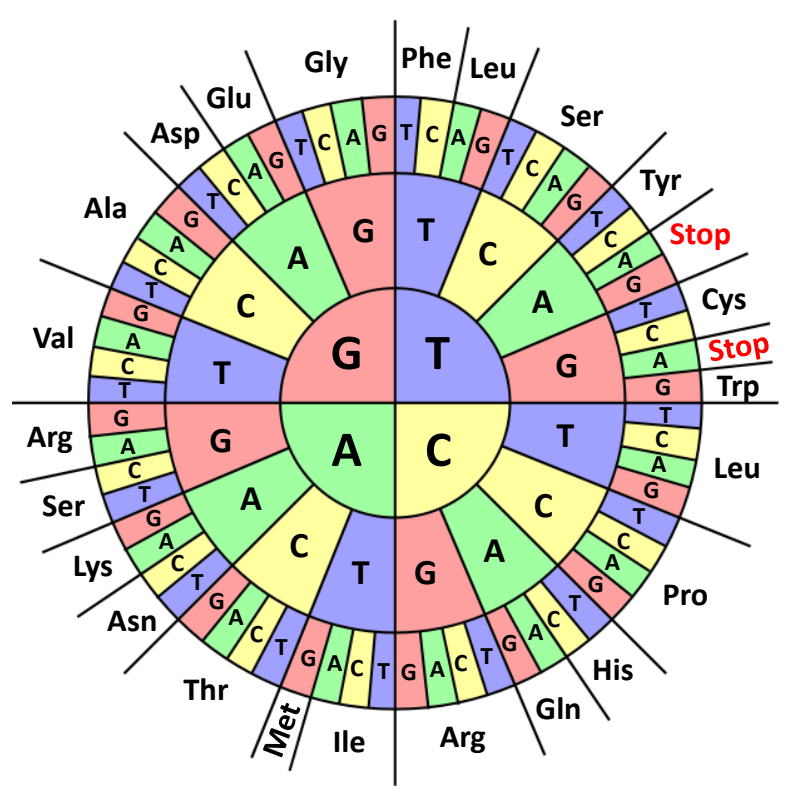

Fig. 2.21 - The Genetic Code

\subsubsection{From proteins to biological functions}

In this section, focus is put on proteins which are the main actors of biological functions. Basically, a biological function is induced by the binding of a protein with other molecules. This mechanism is described in the first section. The second and the third parts describe antibodies and enzymes which are specific proteins. Finally, the two last sections deal with regulations that may occur in metabolic pathways (regulation of the rates of chemical reaction) and/or at the transcriptional level (regulation of the expression of genes). 


\subsubsection{Protein-ligand binding}

The shape of a protein is very important in biochemistry because it mostly determines the protein's biological function. From a macroscopic perspective, the shape of proteins can be highly diversified, but most of them exhibit at their surface specific amino acids sequences that form binding sites. Those binding sites have a shape and local electrostatic properties (e.g. positive or negative charges, propensity for hydrogen bonds ...) in order to bind small molecules called ligands. The analogy that is commonly used to represent these binding sites is that of the lock and the key. A given protein may have several binding site, each of them corresponding to the same or different ligands.

Protein-ligand binding is a natural and instantaneous phenomenon that occurs as soon as the protein and the ligand are put together in a solution. However, the reaction is not always total and there are different ways to quantify its efficiency. At the macroscopic level, we consider that only a given ratio $\theta$ of sites are occupied and that this ratio depends on the ligand concentration and an affinity parameter $K_{A}$. From a microscopic perspective, binding and unbinding occurs stochastically during time with a given probability and get balanced around the ratio $\theta$.

Another crucial notion about protein-ligand binding is the conformational change (allostery). We have to keep in mind that proteins are flexible and reach a shape that minimizes their internal energy. When a binding site is occupied by a ligand, the distribution of energy may locally change and the shape of the rest of the molecule may also change in turn. In particular, other binding sites may be affected, thus increasing or decreasing their affinity with the ligand. Allostery of proteins give a natural way to control the affinity of binding sites for one molecule by the concentration of other molecules.

The mechanism is illustrated of Figure 2.22 on the protein that exhibit 4 binding sites, two for the ligand $\mathrm{L}_{1}$, one for a ligand $L_{2}$ and one for a ligand $L_{3}$. Positive cooperativity occurs between both $L_{1}$ binding site. When a binding site for $L_{1}$ is occupied, the shape of the other binding site for $L_{1}$ change, increasing its affinity (Figure 2.22B). Negative cooperativity occurs between $L_{1}$ and $L_{2}$ sites. When a binding site for $L_{1}$ is occupied, the binding site for $L_{2}$ also change, decreasing its affinity (Figure 2.22D). When the affinity decreases to almost zero, binding sites becomes competitive. Finally, $L_{3}$ is not affected by the fact that $L_{1}$ is occupied. The binding site are independent (Figure 2.22F). Conformational changes are symmetric and reciprocal. In our example, the increase in affinity of the top $L_{1}$ site when the bottom $L_{1}$ site is occupied is the same as that of the bottom $L_{1}$ site when the top $L_{1}$ site is occupied (Figure 2.22C). Similarly, the decrease in affinity of the $L_{1}$ binding site when $L_{2}$ is occupied is the same as that of the $L_{2}$ binding site when $L_{1}$ is occupied (Figure 2.22E).

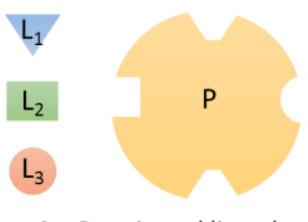

$\boldsymbol{A}$ - Protein and ligands

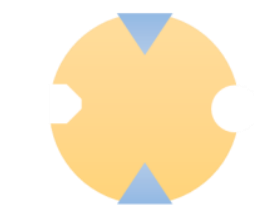

D - both $L_{1}$ site occupied
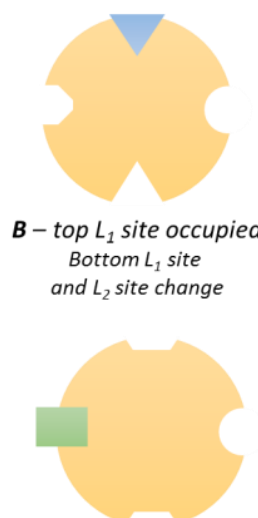

$E-L_{2}$ site occupied Both $L_{1}$ sites change

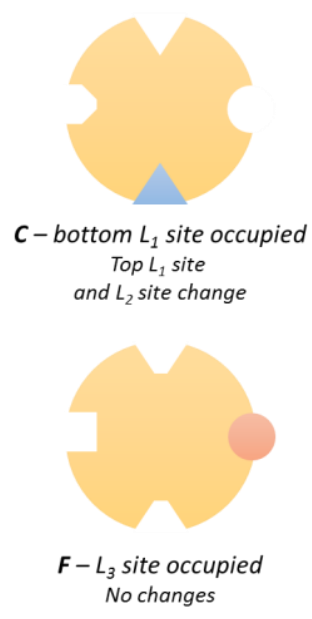


Fig. 2.22 Different conformations of the protein $\mathrm{P}$ depending on the binding of three ligands on protein binding sites.

\subsubsection{Antibodies and antigens}

Antibodies are proteins used for the recognition of specific small amino acid sequences called antigens. The antibodies are Y-shaped (Figure 2.23A) and are composed of four polypeptic chains: two heavy chains (HC) and two light chains (LC) interconnected by disulfide bridges. Each chain is composed of different fragments: 3 or 4 constants and 1 variable for the heavy chains, 1 constant and 1 variable for light chains. The antigen that the antibody can recognize is determined by the two variable fragments. The binding between an antibody and an antigen leads to an immune complex mainly composed of hydrogen bonds. In practice, an antigen may have multiple binding sites (epitopes) for multiple antibodies.

There are several types of antibodies. The first distinction that is made is between the monoclonal antibodies and the polyclonal antibodies. Monoclonal antibodies detects only one single epitope of an antigen whereas polyclonal antibodies is a mixture of antibodies able to recognize multiple epitopes on the same antigen (Figure 2.23B and 2.23C). The name of antibodies often ends by the suffix -mab or -pab. A complete nomenclature of antibodies has been established in 2008 in order to assign a unique and generic name to a given antibody as a function of its target (bacterium, fungus, immune system ...) and its source (human, mouse, primate ...).

Antibodies are used by living cells as key molecules for the immune response according to different mechanisms [43]. For example, different types of antibodies are present near or at the surface of immune cells in order to detect and fix toxins from pathogenic bacteria, preventing them from harming. Similarly, free antibodies can recognize specific antigens at the surface of pathogenic bacteria, bind to them with their variable side and bind in turn to macrophages (cells responsible for the digestion of foreign bacteria) with their fixed side.

Antibodies are also widely used in biotechnology. Their modular structure provide an easy way to detect and fix various proteins with a high level of selectivity. For instance, they are used in biosensors to fix proteins on a surface for further analysis (e.g. in piezoelectric biosensors, antibodies are spotted at the sensor surface and fix protein from the reaction chamber which lead to weight changes measured by the piezoelectric plate) [44]. Other application of antibodies are flow cytometry to differentiate cells [45] or in several experimental protocols to sort proteins of interest from a solution containing many different molecules [46].

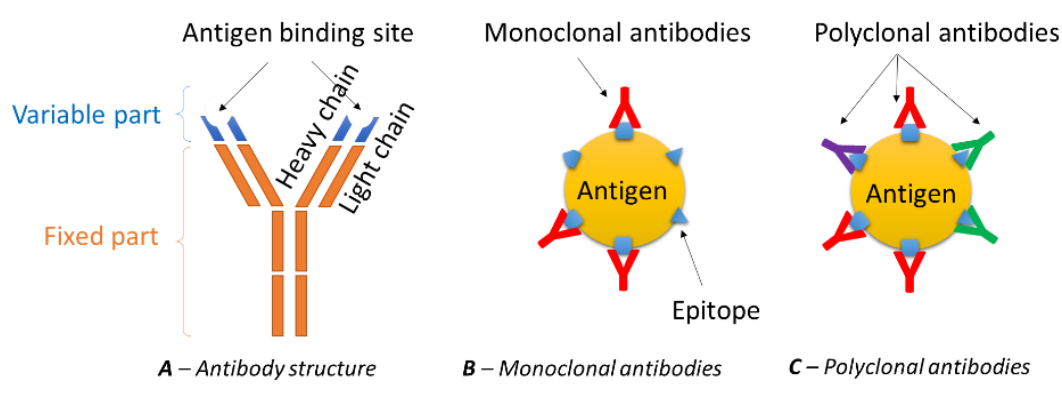

Fig. 2.23 - Structure of an antibody (A) and distinction between monoclonal and polyclonal antibodies (B and C) 


\subsubsection{Enzymes}

Enzymes are another class of proteins that catalyzes chemical reactions. The name of enzymes always ends by the suffix -ase and the radix is often related to the substrate or the product of the reaction they catalyze. To understand the action of an enzyme in a chemical reaction, let us first consider a reaction converting a substrate $S$ into a product $\mathrm{P}$. Let $G_{P}$ and $G_{S}$ be the free energy of the product and the substrate. The reaction occurs only if the energetic balance $\Delta G^{o}=G_{S}-G_{P}$ is positive. But consider now that the conversion from $\mathrm{S}$ to $\mathrm{P}$ involves an intermediate states with free energy $G_{I}>G_{S}$. The difference $G_{I}-G_{S}$ is the activation energy $E_{A}$, i.e. the amount of energy we need to invest in the reaction to go over the energy barrier (Figure 2.23). If $E_{A}$ is high, the reaction will be very slow. It can be compared to a rock on a mountain. The rock would like to go down the mountain in order to decrease its potential energy. But if somewhere it is held by a rim, it will never reach the valley, except if someone gives it enough energy to go over the rim by pushing it.

The role of the enzyme is to provide another intermediate states with a lower and activation energy $E_{A}^{\prime}$. As $E_{A}^{\prime}<E_{A}$, the reaction is faster. Continuing the analogy with the rock, the enzyme creates another pathway from the top of the mountain to the valley on which rims are lower.

From a chemical point of view, enzyme act on the substrate like a nutcracker on a nut. Substrate and enzyme binds together to form a complex in which the transformation from the substrate to the product requires less activation energy in comparison to the direct transformation without complex.

Side chemical species may also be involved in the mechanism. Some of them are part of the reaction by providing or harvesting energy (ATP, see section 2.3.1.4), atoms or functional groups. Some other are not consumed nor produced by the reaction but they improve the efficiency of the enzyme. These chemical compounds are called cofactors or coenzymes [47]. As a conclusion, it should be reminded that enzyme only speed up the reaction. It affects kinetics but not the energy balance of a given reaction.

\subsubsection{Metabolic pathways and regulation}

A metabolic pathways is a set of chemical reactions achieving a specific biological function. Such pathway includes substrates and products (starting and ending points of the pathway), intermediate species, enzymes and regulations. One of the most studied metabolic pathways is the glycolysis which transforms glucose into pyruvate (Figure 2.24). Glycolysis involves 9 intermediate molecules, requires 10 enzymes and 2 cofactors and produces 2 ATPs from 2 ADPs ( 2 ATPs are consumed in the fists steps but 4 are harvested in the last ones).

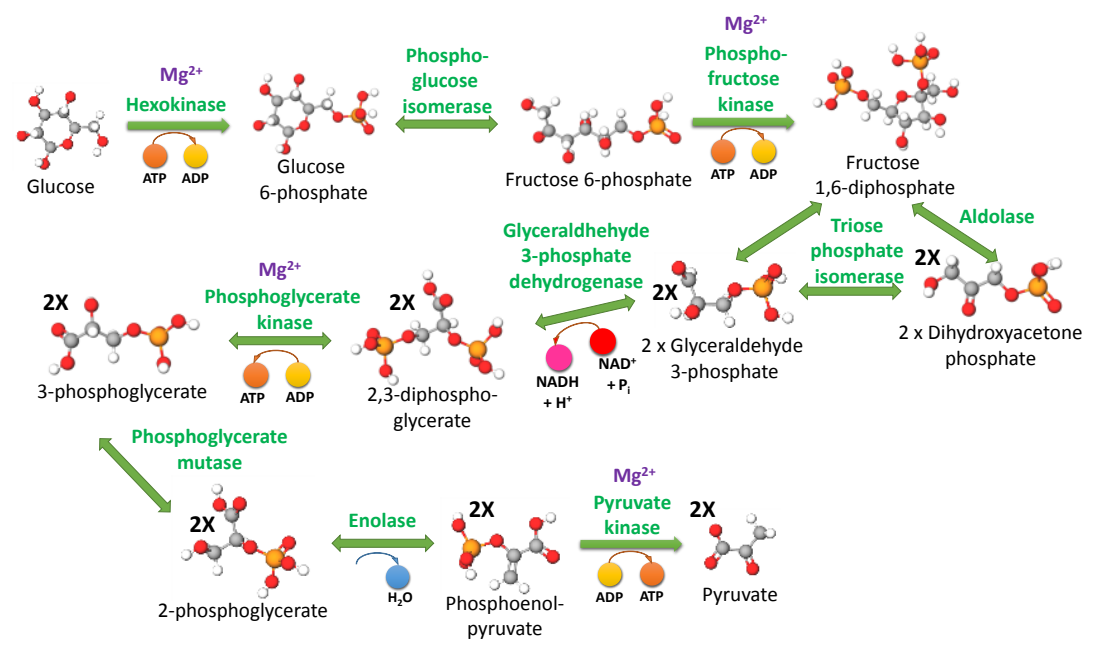

FIG. 2.24-The glycolysis pathway 
Let us use this metabolic pathway to illustrate the role of the ATP and the effect enzymes. Firstly, focus is put on the first reaction of the pathway: the conversion of the glucose into glucose 6-phosphate (G6P). The equilibrium is reached when the concentration of the substrate and the product reach the following equation:

$$
\Delta G^{0}=-R \cdot T \cdot \ln \left(\frac{[P]}{[S]}\right)
$$

where $R$ is the gas constant $\left(R=8.31 \mathrm{~J} \cdot \mathrm{K}^{-1} \cdot \mathrm{mol}^{-1}\right), T$ is the temperature and $\Delta G^{0}$ is the standard free energy of the reaction, which is $14.3 \mathrm{~kJ} \cdot \mathrm{mol}^{-1}$ for the glucose $+\mathrm{H}_{3} \mathrm{PO}_{4} \rightarrow \mathrm{G} 6 \mathrm{P}$. As a consequence, at ambient temperature, at equilibrium the glucose concentration is about 300 time higher than G6P concentration. To alter this equilibrium the reaction, it is coupled to an ATP hydrolysis (ATP $+\mathrm{H}_{2} \mathrm{O} \rightarrow$ ADP $\left.+\mathrm{H}^{+}+\mathrm{H}_{3} \mathrm{PO}_{4}\right)$ which $\Delta G^{0}$ is $-30.5 \mathrm{~kJ} \cdot \mathrm{mol}^{-1}$. The overall $\Delta G^{0}$ of the coupled reaction is now $-16.7 \mathrm{~kJ} \cdot \mathrm{mol}^{-1}$ which lead to glucose concentration at equilibrium 1000 time lower than the concentration of G6P.

Second, focus is put on another reaction of this pathway: the isomerization (same chemical formula but different atomic configuration) of glyceraldehyde 3-phosphatye (GAP) into dihydroxyacetone phosphate (DHAP). The reaction consists in two hydrogens transfer (Fig. 2.25) with the cis-enediol as an intermediate state. The reaction balance is $\Delta G^{0}=-7.8 \mathrm{~kJ} / \mathrm{mol}$ but the free energy of cis-enediol is very high $\left(E_{A}=100 \mathrm{~kJ} / \mathrm{mol}\right)$. As a consequence, the reaction may occur but will be very slow. GAP isomerization can be catalyzed by an enzyme, the triose phosphate isomerase (TIM), which speed up the reaction by a factor $10^{10}$. In practice, the GAP binding site of TIM exhibits an $\mathrm{H}^{+}$arm on the right and a negatively charged arm one on the left. When GAP binds on TPI, the two hydrogens that need to be transferred connect to these arms. In terms of energy, we are at the point $C$ on the curve in Figure 2.25E, which is obviously a non-stable equilibrium state. When the molecule is release, DHAP is preferred rather than GAP because its free energy is lower. According to equation (2), at equilibrium, there are 22 times more DHAP than GAP.
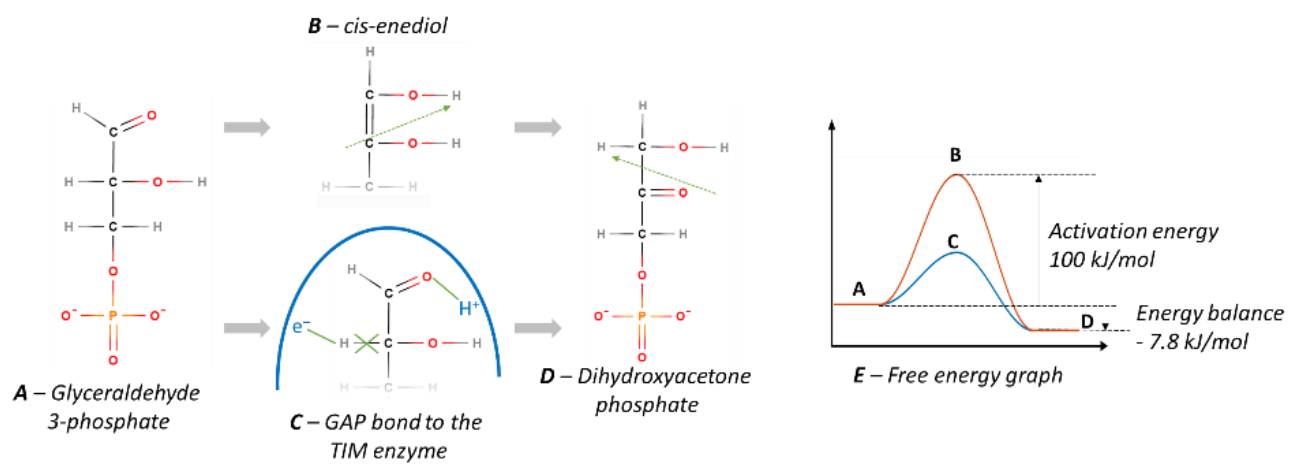

Fig. 2.25 Illustration of the enzymatic activity on the reaction glyceraldehyde 3-phosphate $\rightarrow$ Dihydroxyacetone phosphate.

Metabolic pathways are not always active and cells have different ways to regulate them. First, it must be remembered that a reaction is never complete and the concentration ratio between substrate and products is fixed by energetic considerations (Eq. 2.39). Thus, a metabolic pathway can be blocked as soon as the concentration of a product reached the equilibrium. This natural mechanism prevents from an excessive accumulation of a products. Another way to increase/decrease enzyme's activity is to use cofactors. For instance, the concentration of $\mathrm{Mg}^{2+}$ increase the activity of several enzymes in glycolysis pathway (e.g. hexokinase, see Fig. 2.23). Moreover, most of the metabolic pathways are self-regulated with 
feedback loops. For instance, G6P is an inhibitor for hexokinase. It induces a conformational change that reduce the affinity of the glucose binding site of hexokinase.

The number of possible regulation and, as a consequence, the complexity of the pathway increase dramatically with the number of steps and the number of molecules involved in the pathway.

\subsubsection{Transcriptional regulation}

Another way to regulate metabolic pathways is to control directly the transcription of genes that encode for the enzymes involved in the pathway. The principle of DNA-level regulation is the same as for metabolic regulation except that the regulation molecule controls the binding rate of the RNA polymerase to DNA instead of an enzyme activity.

In practice, there is a specific DNA sequence called the promoter upstream of the gene. RNA polymerase may bind on this promoter to initiate the transcription. Several mechanisms are implemented to regulate this transcription. First, some molecules have the appropriate conformation in order to bind to the DNA on or just after the promoter, preventing the binding of RNA polymerase and the transcription. Then regulatory DNA sequences can also be found before the promoter. Those sequences form binding site for regulating molecules (transcription factors) which modify the promoter structure and increase/decrease its affinity for RNA polymerase. Sometimes, the regulation mechanism is more complex. For instance, the human insulin gene can be regulated by up to 14 transcription factors [48].

To illustrate the concept, the example of the lactose operon is described hereafter. Lactose operon provides bacteria with a mechanism to digest and metabolize the lactose (Fig. 2.26). The operon has been first described in 1961 by Jacob and Monod, which is considered as the first breakthrough in the understanding of gene regulation mechanisms [49]. The Lac operon is composed of two parts: first, a constitutive promoter (always active) and a gene coding for Lacl and second, a regulated promoter and three genes coding for LacZ (coding for $\beta$-galactosidase), LacY (coding for $\beta$-galactosidase permease) and LacA (coding for galactoside O-acetyltransferase) on the right. The idea of the regulation is the following: if there is no lactose around the bacteria, it is a waste to produce the enzymes letting lactose enter into the cell and metabolizing it. As a consequence, the gene expression should be repressed. The repression mechanism is performed by the Lacl protein which binds to the Lac promoter, preventing the RNA polymerase from transcribing the genes. Conversely, if there is a large amount of lactose molecules around the cell, some of them penetrate into the bacteria and bind Lacl. This induces a conformational change that prevents Lacl from binding DNA. As a consequence, genes are expressed: LacY opens channels allowing more lactose to invade the cell and LacZ breaks lactose into glucose and galactose. When there is no more lactose to consume, the last molecules of lactose unbind from Lacl which is able to repress gene transcription again.

Moreover, glucose remains the preferred energy source for bacteria. As a consequence, in presence of glucose, lactose metabolism is not required. Lactose operon is thus also repressed by glucose (not represented in Fig. 2.26). 


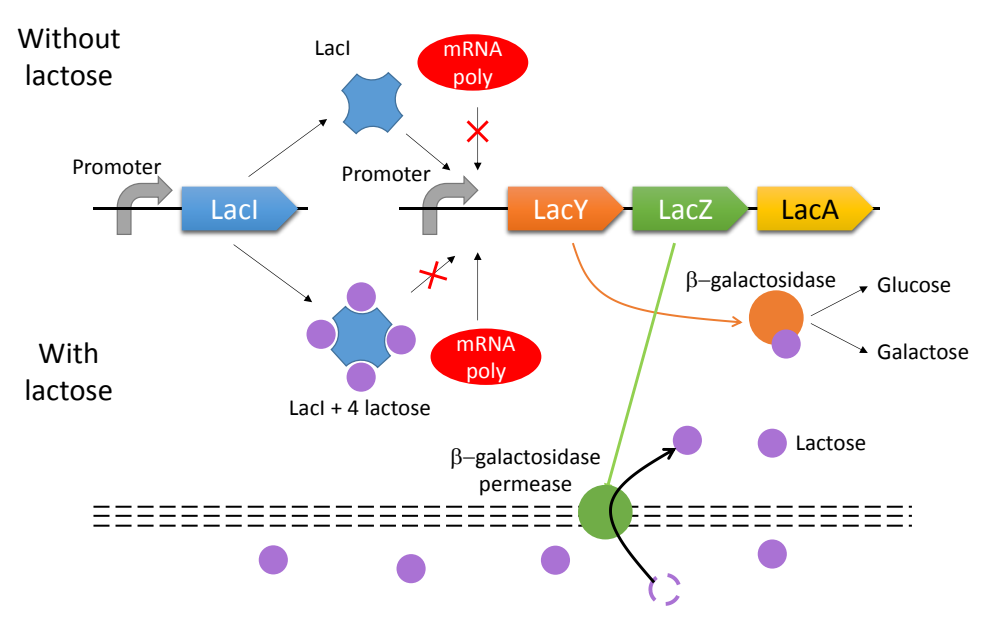

Fig. 2.26 - The Lacl operon

Gene regulation can be cascaded (operons are constituted of genes that codes for transcription factors regulating other operons) in order to achieve more complex biologica functions. In this case, we speak about gene regulatory networks. Moreover, regulatory network also exists at translational level: regulatory molecules modify the mRNA translation rates.

\subsubsection{Mathematical modeling}

The last part of this sub-chapter aims at giving the background on mathematical modeling of the biological mechanisms described here above.

\subsubsection{Generic framework for a biological system}

A biological system is a set of chemical compound linked by reactions. To describe it in a mathematical way, four elements are requires. Firstly, the molecules involved in the system are listed. Let $\mathbf{X}(t)$ be an $N$ element vector composed of the concentration of the $N$ involved molecules. Secondly, the reactions are also listed. Let $\mathbf{V}(t)$ be an $M$-element vector composed of the rates of the $M$ reaction. Because of regulations, the elements of $\mathbf{V}$ are not constant but depends on some elements of $\mathbf{X}$. Thirdly, a stoichiometry matrix is build. It gives the number of molecule produced or consumed by each reaction. Let $\mathbf{S}$ be this $N \times M$ matrix. Finally, most of the molecules of living matter are not stable over time and degrade with a given ratio. Thus, we also define the decay vector $\mathbf{d}$, an $N$-element vector composed of the decay constant for each involved molecule.

A first way to simulate the system is to compute the temporal evolution of the concentration of each species. At each time step, the variation of concentration for each species is equal to the sum of the production, consumption and degradation rate. From the quantities define here above, we can write the set of ordinary differential equation (ODE) to solve in a vector form:

$$
\frac{d \mathbf{X}}{d t}=\mathbf{S} \times \mathbf{V}(\mathbf{X})-d \cdot \mathbf{X}
$$

Such equation rate can be solved with standard ODE solver (such as Matlab, SciLab, Pyhton), with dedicated tools such as COPASI [50] but also with SPICE-like simulators [51]. An alternative way to analyze this system is to find the steady states which correspond, for each species, to a balance between synthesis rate, consumption rate and decay rate. Steady states can be obtained by solving the following equation set:

$$
\mathbf{S} \times \mathbf{V}(\mathbf{X})-d \cdot \mathbf{X}=0
$$

The number species and mechanisms to take into account in an actual biological system can be very large, which often leads to large and strongly coupled ODE sets. Simulations require long computation time and 
lead to convergence issues. There are some tricks to simplify the model of large systems. The most usual one consists in representing the system as an interaction graph and use graph theory to identify poles that corresponds to subsystems for which high-level macro-models can be used. The approach is exactly the same as for multi-level modeling in microelectronics (e.g. in electronic circuit, a behavioral description for operational amplifier instead of a transistor-level model). For instance, let us consider a complex system involving glycolysis. If enzymes and intermediate species involved in glycolysis do not interact with the rest of the system, the full pathway can be modeled by a single equation giving the production rate of pyruvate as a function of the concentration of glucose and the quantity of enzyme (supposed to be constant). This macro-model allow to get rid of 9 species and 10 reaction in comparison with the complete model.

Another way to simplify the equation set is to use the quasi steady state approximation (QSSA). QSSA can be applied as soon as several mechanisms are very fast in comparison to other ones. In this case, the fastest reaction are considered to be always "almost at equilibrium", i.e. between two time steps of the slowest reaction, the fastest one has reached the steady state. QSSA will be illustrated in subsection 2.3.3.4.

\subsubsection{First order reactions}

In the following four subsections, the most common models for reaction rates are described. For a standard chemical reaction, such as $A+B \leftrightharpoons C+D$ the most common model is the first-order model. The rate of the reaction is given by:

$$
v=k_{o n} \cdot[A] \cdot[B]-k_{o f f} \cdot[C] \cdot[D]
$$

where $k_{\text {on }}$ and $k_{\text {off }}$ are the forward and reverse rate constant. Equilibrium is reached when the reaction rate is zero. In this case, association constant $K_{A}$ and dissociation constant $K_{D}$ can be defined as following:

$$
K_{A}=\frac{1}{K_{D}}=\frac{k_{o n}}{k_{\text {off }}}=\frac{[C] \cdot[D]}{[A] \cdot[B]}
$$

\subsubsection{Hill's equation}

In this subsection, we consider a reaction involving a macromolecule $\mathrm{P}$ that may bind up to $N$ ligands $\mathrm{L}$. The system can be described by a set of first-order reaction $P+L \rightarrow P L, P L+L \rightarrow P L_{2}, P L_{2}+L \rightarrow P L_{3}, \ldots$ Let $K_{A_{1}}, \ldots K_{A_{N}}$ be the macroscopic association constant for each of these reaction. The total quantity of $\mathrm{P}$ is distributed among the different potential configurations:

$$
P_{\text {TOT }}=[P]+[P L]+\cdots=[P] \cdot\left(1+K_{A_{1}} \cdot[L]+K_{A_{1}} \cdot K_{A_{2}} \cdot[L]^{2} \cdots\right)
$$

This expression is named the binding polynomial. We then associate a signal $\sigma_{k}$ to each configuration of $\mathrm{P}$. This signal can be either a reaction rate, a transcription rate for a promoter, the average number of occupied binding site, a fluorescence signal, etc. The signal polynomial is defined as following:

$$
S=[P] \cdot\left(\sigma_{0}+\sigma_{1} \cdot K_{A_{1}} \cdot[L]+\sigma_{2} \cdot K_{A_{1}} \cdot K_{A_{2}} \cdot[L]^{2} \ldots\right)
$$

The total signal associated to the protein $\mathrm{P}$ is given by the rational fraction:

$$
S_{T O T}=\sum_{i=0}^{N} \sigma_{i} \cdot \frac{\left[P L_{i}\right]}{P_{T O T}}=\frac{S}{P_{T O T}}
$$

This approach can be extended to macromolecules with multiple binding sites for multiple ligand and the associated signal can always be written as a rational fraction [52].

Let us discuss about some simple cases. First, consider that $\mathrm{P}$ has a single binding site with the macroscopic dissociation constant $K_{D}$. The signal of interest is the average number of occupied sites $\theta$. From equation (2.3.9): 


$$
\theta=\frac{K_{A_{1}} \cdot[L]}{1+K_{A_{1}} \cdot[L]}=\frac{1}{1+\frac{K_{D}}{[L]}}
$$

Now consider that $\mathrm{P}$ has $N$ equivalent binding sites. The average number of occupied sites is now given by:

$$
\theta=\frac{\sum_{i=1}^{N} i \cdot \prod_{j=0}^{i} K_{A_{j}} \cdot[L]^{j}}{1+\sum_{i=1}^{N} \prod_{j=0}^{i} K_{A_{j}} \cdot[L]^{j}}
$$

where $K_{A}$ is the affinity constant of binding site when $j-1$ other sites are occupied. If binding sites are equivalent and independent (i.e. $K_{A_{j}}=k_{A}^{j}$ ), it has been demonstrated that [52]:

$$
\theta=N \cdot \frac{k_{A} \cdot[L]}{1+k_{A} \cdot[L]}=\frac{1}{1+\frac{k_{D}}{[L]}}
$$

where $k_{A}$ and $k_{D}$ are the microscopic affinity for one each binding site. Comparison between equation (2.46) and (2.48) highlights the fact that multiple binding independent binding sites behaves as one single binding site.

Now, let us consider that binding sites are cooperative, i.e. the affinity of binding site increases with the number of occupied sites. From a mathematical point of view, strong cooperativity leads to the following relationship: $K_{A_{i}} \gg K_{A_{i+1}} \cdot[L]$. In other words, binding and signal polynomial are dominated by the zeroorder term and the highest-order term. Thus, the average number of occupied sites is given by:

$$
\theta=N \cdot \frac{\left(\prod_{i=0}^{N} K_{A_{j}}\right) \cdot[L]^{N}}{1+\left(\prod_{i=0}^{N} K_{A_{j}}\right) \cdot[L]^{N}}=\frac{1}{1+\left(\frac{K_{D^{\prime}}}{[L]}\right)^{N}}
$$

where $K_{D}{ }^{\prime}$ is the apparent dissociation constant. Equation 2.49 is the Hill equation. In practice, cooperativity is not absolute and the assumption used to deduce equation (2.49) is not completely met. But most of the time, a binding mechanism with cooperative sites exhibits a sigmoid response and can be modeled by a Hill equation with empirical Hill constant $K_{D}{ }^{\prime}$ (concentration of $[L]$ for which half of the sites are occupied) and Hill number (between 1 and the number of sites) [53]. To illustrate the purpose, Fig. 2.27 shows examples of $\theta([L])$ curves for 4 binding sites with weak and strong cooperativity.
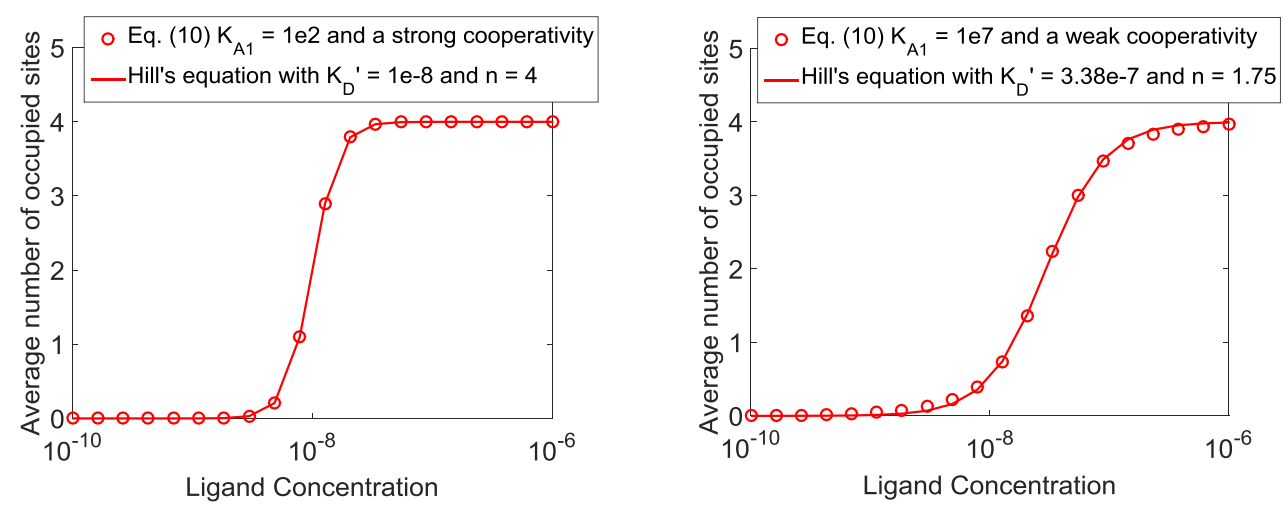

Fig. 2.27 Modeling multi-site cooperativity in protein-ligand binding with Hill's equation

Hill's equation is a universal model that can be applied for most of the signal of interest that are proportional to the site occupancy rate. 


\subsubsection{Michaelis and Menten's enzymatic model}

As described in section 2.3.2.3 an enzymatic reaction can be decomposed into two stages: the binding of the substrate on the enzyme $(E+S \rightarrow E S)$ and the transformation of the enzyme-substrate complex into the product and the recycled enzyme $(E S \rightarrow E+P)$. Each reaction can be modelled by a first-order reaction rates. Let $k_{1}$ and $k_{-1}$ be respectively the forward and reverse reaction constant for the first reaction and $k_{2}$ and $k_{-2}$ for the second. Let us model the complete enzymatic reaction according to the formalism described in section 2.3.3.1. The species vector, reaction rate vector and stoichiometry matrix are the following (decay is neglected and to simplify writing, square brackets around concentration have been omitted):

$$
\begin{aligned}
& \mathbf{X}=\left[\begin{array}{c}
E \\
S \\
E S \\
P
\end{array}\right] \quad \mathbf{V}=\left[\begin{array}{l}
k_{1} \cdot E \cdot S-k_{-1} \cdot E S \\
k_{2} \cdot E S-k_{-2} \cdot E \cdot P
\end{array}\right] \quad \mathbf{S}= \\
& {\left[\begin{array}{cc}
-1 & 1 \\
-1 & 0 \\
1 & -1 \\
0 & 1
\end{array}\right] \quad(2.50)}
\end{aligned}
$$

The Michaelis-Menten model relies on two assumptions: i) the second reaction is not reversible $\left(k_{-2}=0\right)$ and ii) the second reaction is much faster than the first one. As a consequence, QSSA assumption can be applied on $E$ and $E S$. Each time an $E$ is consumed and an ES is produced by reaction 1, an ES is consumed and an $E$ is produced by reaction 2 . As a consequence, the time derivative of the concentration of $E$ and $E S$ are equal to zero. From (2.50), one can write:

$$
\frac{d[E S]}{d t}=-\frac{d[E]}{d t}=k_{1} \cdot[E] \cdot[S]-\left(k_{-1}+k_{2}\right) \cdot[E S]
$$

Thus,

$$
k_{1} \cdot[E] \cdot[S]=\left(k_{-1}+k_{2}\right) \cdot[E S]
$$

Let $E_{T O T}$ be the total amount of enzyme $\left(E_{T O T}=[E]+[E S]\right)$. It can be deduced from (2.51) and (2.52) that the production rate of $\mathrm{P}$ is equal to the consumption rate of $\mathrm{S}$ which is also equal to:

$$
\frac{d[P]}{d t}=-\frac{d[S]}{d t}=\frac{v_{\max } \cdot S}{K_{m}+S}
$$

where $v_{\max }=k_{2} \cdot E_{T O T}$ is the maximal reaction rate (when $S$ is high) and $K_{M}=\frac{k_{-1}+k_{2}}{k_{1}}$ is the Michaelis constant. Figure 2.28 shows simulation results for enzymatic reaction with complete model and with Michaelis-Menten model for different cases: one in which QSSA condition is met (simulation results fit perfectly), one in which it is almost met and one in which it is not met (error appear between the two curves).

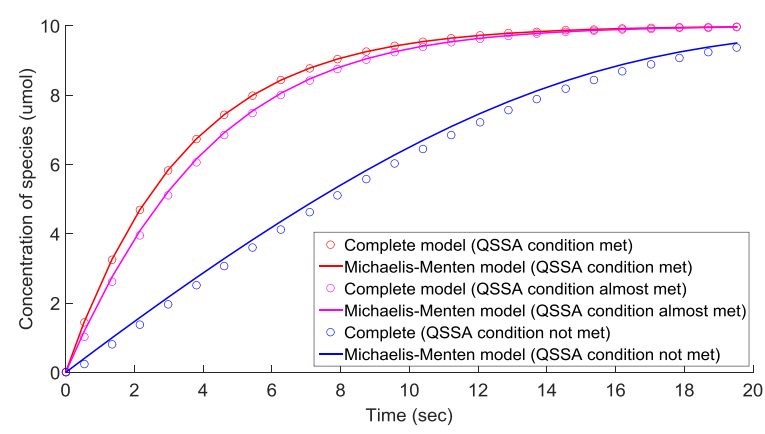

Fig. 2.28 Comparison of simulation results between complete model and michaelis-menten model 
Again, if an enzymatic reaction involved in a larger pathway pas be described by a unique reaction $S \rightarrow P$ with a Michaelis-Menten model as soon a neither E nor ES, interact with the other molecules involved in the pathway.

\subsubsection{Transcription and translation}

Without regulation, the transcription rate of DNA into mRNA mostly depends on the efficiency of RNA polymerase. Most of the time in model, the concentration of RNA polymerase is considered to be high enough and does not impact the transcription rate $k_{t r}$ which is a constant depending only on the length of the sequence. In practice, this assumption remains questionable. By the same way, the translation rate depends on the efficiency of the ribosome which is also considered to be in excess. The translation rate of one mRNA into a protein is given by a constant $k_{t l}$ which also depends on the sequence length. As several copy of the same mRNA may exist and may be translated in parallel, the overall translation rate is given by $k_{t l} \cdot[m R N A]$.

DNA transcription and mRNA translation are very slow mechanism in comparison to molecule binding (timescale for the binding of transcription factor to DNA site is about 1 second whereas timescale for transcription and translation is more than 1 minute [54]. As a consequence, regulation can be introduced in transcription/translation models under the QSSA approximation. Let us first consider a promoter with a regulating sequence with four cooperative binding site for a transcription factor $A$. We assume that the transcription rate increases monotonically with the number of occupied binding site so that the Hill's equation can be applied. QSSA condition allows to modulate directly the transcription rate with a term depending on the concentration of $A$ :

$$
k_{t r}=k_{t r, \text { max }} \cdot\left(\alpha+\frac{1-\alpha}{1+\left(\frac{K_{A}}{[A]}\right)^{n_{A}}}\right)
$$

Where $\alpha(\ll 1)$ is the promoter leakiness (even if the promoter is repressed, a basal transcription rate exists), $k_{t r, \max }$ is the maximal transcription rate, $K_{A}$ and $n_{A}$ are Hill's parameters and number. $K_{A}$ is sometimes called activator strength because it represent the concentration of activator required to reach about $50 \%$ of the maximal transcription rate. In the case of a repressor, transcription rate decreases with the increasing site occupancy ratio. As a consequence, the expression becomes:

$$
k_{t r}=k_{t r, \max } \cdot\left(\alpha+\frac{1-\alpha}{1+\left(\frac{[R]}{K_{R}}\right)^{n_{R}}}\right)
$$

where $K_{R}$ and $n_{R}$ play the same role as $K_{A}$ and $n_{A}$ for the activator. Expression (2.54) and (2.55) can be merged for promoter with more than one transcription factor. Same principles can be applied to the translation rate for RNA-based regulation system.

\subsection{MicroPIV measurement technique (Denis Funfshilling, Norbert Dumas)}

\subsubsection{Introduction}

Among the different measurement techniques existing in microfluidics, two techniques have emerged: imaging (direct imaging, fluorescence imaging, shadow imaging and the following image analysis) and $\mu$ PIV (Micro Particle Image Velocimetry) technique for flow field measurements. They both are valuable source of information for experimentalist. This chapter is focused on this latter technique. The origin of the $\mu$ PIV dates back to the pioneer work of Santiago et al. 1998 [55] and Wereley et al. 1998 [56]. $\mu$ PIV is considered as the most widespread method for measuring velocities in fluids [57]. 
The $\mu$ PIV (Micro Particle Image Velocimetry) is an adaptation of already existing PIV technique to the microscale. The spatial resolution of a $\mu$ PIV can be better than $1 \mu \mathrm{m}$ [58]. It has the enormous advantage to measure the flow field in a complete plane, not like other techniques like LDV (Laser Doppler Velocimetry), or Ultrasonic Doppler Velocimetry which operate only point wise or line wise. In short, the PIV technique consists of (1) seeding the fluid with particles, (2) illuminating the fluid with a laser sheet (most of the time, a double cavity Yag Laser is used), (3) taking two images separated by a known interval of time, more precisely, each image is taken during the burst of the laser that lasts a few nanoseconds, (4) both images are divided into interrogational areas, and each of these interrogational areas is cross-correlated with the corresponding interrogational areas of the second image, (5) the mean velocity on each interrogational area is deduced by dividing the mean displacement of the particles of this area by the time between the two bursts. But unlike the PIV where the velocity field is measured in a plane created by a laser sheet obtained by expanding the laser beam in 2D by a cylindrical lens, the $\mu$ PIV uses the depth of field of the imaging optics to image a "slice" of the flowing seeded fluid [59].

Several companies such as Dantec Dynamics are proposing complete $\mu$ PIV set-up. (www.dantecdynamics.com), LaVision (www.lavision.de) or TSI (www.tsi.com). MPIV is particularly interesting for biology research in microfluidics because it gives a mine of information about the flow, the stresses, the movement of cells, bacteria...

In some research fields, it has been the major source of development in the last decade, for example:

- Droplet and bubble manipulation is a flagship for microfluidics applications. Droplets have been used as independent chemical and biological reactors for cell culture, protein crystallization, nanoparticles synthesis, enzyme kinetics [60] or biological assays for living cells [61] among others. A deeper understanding of the mechanisms driving the formation and motion of droplets and bubbles is necessary for the effective design of multiphase microfluidic devices [60]. $\mu$ PIV is the most appropriate measurement technique to achieve this goal.

- Growth and evolution of cells like endothelial cell under flow can be measured by $\mu$ PIV in microsystems [62].

- In the rheology domain, Rodd et al. 2007 [63 think that $\mu$ PIV in micro channels is the most effective technique for reliably and quantitatively characterize the kinematics of complex flow over a wide range of flow regimes.

\subsubsection{Preliminary characterization of fluids and microsystems}

Before beginning experiments in microsystems, experimentalists must collect the physical properties of the fluids used, like viscosity, density, surface tension, interfacial tension as well as the surface properties of the microchannels' walls. When pure fluids are used, these properties are most of the time well documented in books like CRC Handbook of Chemistry and Physics, Taylor \& Francis Group [64]. Otherwise, when mixtures of fluids are used, in lab measurements may have to be undertaken.

\subsubsection{Density}

The density of a fluid can be measured by a pycnometer (a glass beaker of a known volume filled with the fluid and weighted on a high precision scale) or by a hydrometer. A hydrometer is represented schematically on Fig. 2.29. The measurement is based on the Archimedes principle. A calibrated glass body dips into the sample. The part of the graduated glass emerging from the fluid depends on the density of the fluid to be measured. Companies like VWR or Fisher Scientific are selling these inexpensive chemistry lab materials. 


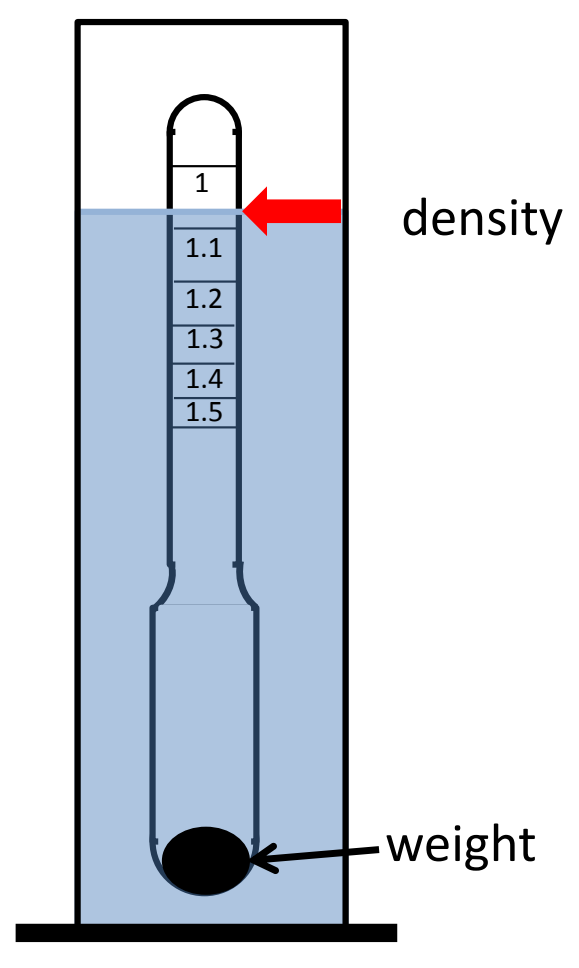

Fig. 2.29 schematic representation of a hydrometer

More elaborate density measurement apparatus are also available like (i) hollow glass tube. The tube is filled with the fluid. The frequency of vibration of the tube is related to the density of the fluid, (ii) radiometric density measurements. The radiometric measurements are non-invasive, non-intrusive and based on gamma-ray or X-ray transmission principle: i.e. the attenuation of the radiation is proportional to the density. Such measurements devices are manufacture for example by Berthold Technologies GmbH (Germany) or Ronan Engineering Company (UK).

\subsubsection{Viscosity}

The first rheological question arising is whether the viscosity of the fluid is Newtonian or non-Newtonian.

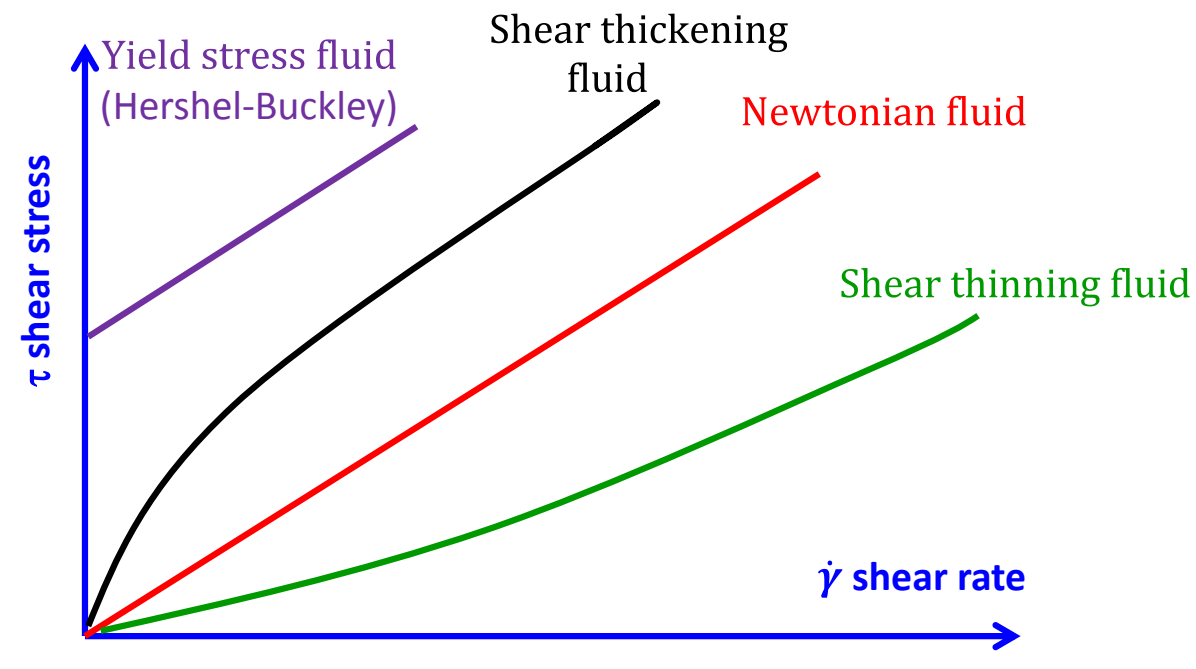

Fig. 2.30: Behaviour of Newtonian and non-Newtonian fluids (shear thickening, shear thinning and yield stress non-Newtonian fluids are represented).

When the fluid is Newtonian, its viscosity is constant, i.e. its viscosity is independent of shear rate and time. As represented in Fig. 2.30, the shear stress is then a linear function of the shear rate, i.e. 


$$
\tau=\mu \gamma^{\prime}
$$

where $\tau[\mathrm{Pa}]$ is the shear stress, $\mu[\mathrm{Pa} . \mathrm{s}]$ the viscosity, and $\gamma=\frac{d U}{d x}\left[\mathrm{~s}^{-1}\right]$ the shear rate.

Most of the pure simple fluids around us are Newtonian (water, alcohol, glycerol, silicone oil...). In this case, the viscosity can be measured in a viscometer. Several affordable viscometers are available like capillary, falling ball or rotational viscometers.

- Capillary viscometer (see Fig. 2.31). This type of viscometer also named Ostwald viscometer is quite common in laboratories and is appreciated for its simplicity, precision and low cost. Its physical principle is based on the measurement of flow resistance (i. e. pressure loss) of the fluid flowing through a capillary.

The fluid is usually flowing under the earth gravitational force. The time necessary for a certain amount of fluid to flow through the capillary is measured. The viscosity is then calculated with the formula:

$$
v=K_{c} \times t_{f}
$$

where $\left[\mathrm{m}^{2} / \mathrm{s}\right]$ is the kinematic viscosity, $K_{c}\left[\mathrm{~m}^{2} / \mathrm{s}^{2}\right]$ is the capillary factor and $t_{f}[\mathrm{~s}]$ the time measured corresponding to the flow of the volume of fluid located between the start and stop marks (see Fig. 2.31).

The previous formula is only valid in the laminar flow regime and is based on the HagenPoiseuille Law i.e. $\frac{V}{t}=\frac{\pi R^{4} \Delta P}{8 L \mu}$, where $R[\mathrm{~m}]$ is the radius of the capillary, $L[\mathrm{~m}]$ the length of the capillary, and $\Delta P[\mathrm{~Pa}]$ the pressure drop through the capillary. Several capillary viscometers with different capillary diameters may be necessary to cover larger ranges of viscosities.

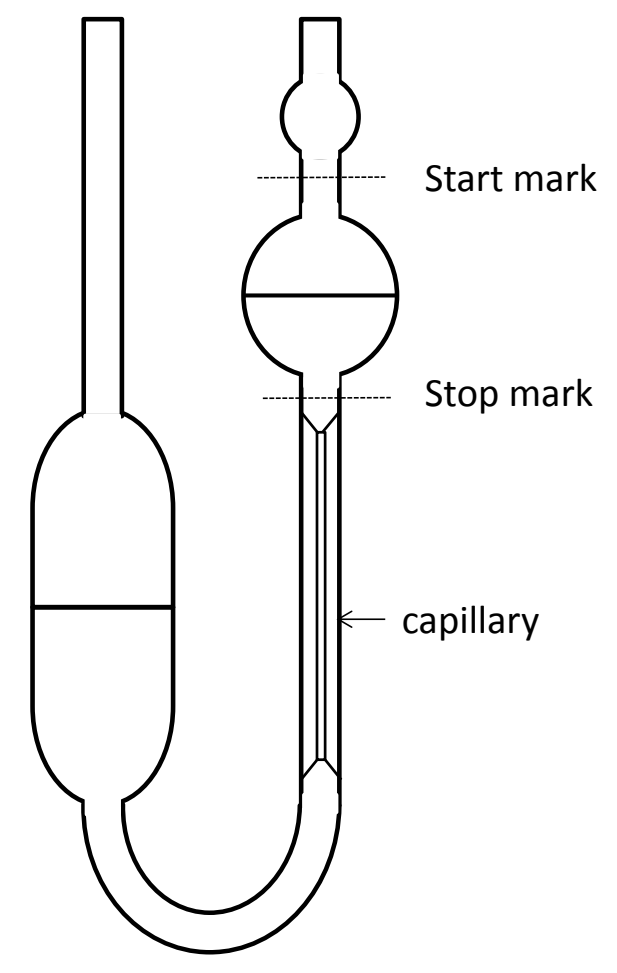

Fig. 2.31: Schematic representation of a capillary viscometer

- Falling ball viscometer. The measurement is based on Newton's law of motion. The force balance refers to a falling ball which has reached its terminal velocity. Three forces are present: the buoyancy force, the weight force and the drag force. Measurement have to be done in the Stokes' flow regime (i.e. Reynolds number $<1$ ). In this case, the drag force on the ball is:

$$
F_{D}=3 \pi \mu u_{t} d
$$

where $d[\mathrm{~m}]$ is the diameter of the ball and $u_{t}[\mathrm{~m} / \mathrm{s}]$ the terminal velocity. 
The viscosity is then:

$$
\mu=\frac{g\left(\rho_{s}-\rho_{f}\right) d^{2}}{18 u_{t}}
$$

Where $\rho_{s}$ and $\rho_{f}\left[\mathrm{~kg} / \mathrm{m}^{3}\right]$ are the densities of the ball and of the fluid respectively, and $\mathrm{g}\left[\mathrm{m}^{2} / \mathrm{s}\right]$ the acceleration of gravity.

- Rotational viscometer. In the case of rotational viscometers, the angular velocity and the torque of a rotating geometry (a disk, a bob, a spindle, a needle, a cylinder or a cup) are measured simultaneously. Knowing the exact dimensions of the geometry, and assuming a laminar flow, it is possible to calculate the viscosity. These kinds of viscosity measurements are well appreciated in industry, because the rotating geometry is directly immersed into the fluid and the reading is immediate. Rotational viscometers are not appropriate for non-Newtonian fluids viscosity measurements because of their limited accuracy and limited range of shear rates available for viscosity measurements. In this case, rheometers are necessary for a more precise and complete investigation of the viscosity of the fluid.

When the viscosity is non-Newtonian (the fluid can be shear thinning, shear thickening, thixotropic (time dependent), have a yield stress....), measurements must be done in Rheometers.

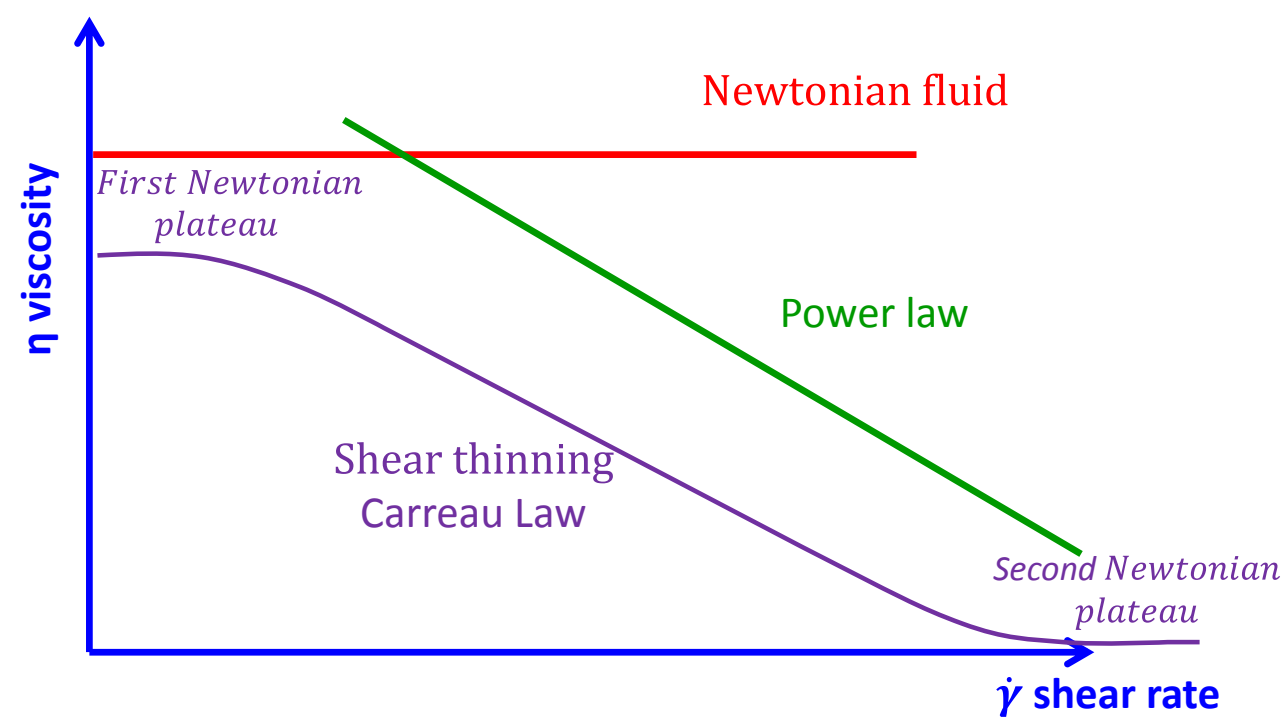

Fig. 2.32 Viscosity of Non-Newtonian fluids following a power law or a Carreau-Yasuda law

Blood and most polymers and polymer solutions are non-Newtonian. The simplest non-Newtonian fluids are fluids whose viscosities can be expressed by a power law (see Fig. 2.32) :

$$
\eta=k \dot{\gamma}^{n-1}
$$

$\eta[\mathrm{Pa} . \mathrm{s}]$ is the viscosity, $k\left[\mathrm{~Pa} . \mathrm{s}^{\mathrm{n}}\right]$ is the consistency index, and $n[-]$ the flow behavior index.

Another simple model for non-Newtonian fluids is the Carreau-Yasuda model:

$$
\frac{\eta\left(\gamma^{\prime}\right)-\eta_{\infty}}{\eta_{0}-\eta_{\infty}}=\left[1+\left(\lambda \gamma^{\prime}\right)^{\frac{n-1}{a}}\right.
$$

$\eta_{0}[\mathrm{~Pa} . \mathrm{s}]$ is the zero shear viscosity corresponding to the first Newtonian plateau, $\eta_{\infty}[\mathrm{Pa}$.s] is the infinite shear viscosity corresponding to the second Newtonian plateau, $\lambda[s]$ is a time constant, $n[-]$ the flow behavior index and $a[-]$ represents the width of the transition region between $\eta_{0}$ and the power-law region. 
Rheometers are necessary for rheology measurements of non-Newtonian fluids. They are expensive and sophisticated devices often used in research and able to measure viscosities in function of time shear rate, dynamic oscillations, or after a step in shear rate.... The main manufacturers are TA Instruments (http://www.tainstruments.com), Anton-Paar (http://www.anton-paar.com), Brookfield http://www.brookfieldengineering.com). They are based on the same principle as the rotational viscometers. They simultaneously measure the angular velocity and the torque. The most common geometries are cone and plate, plate and plate, Couette or double Couette cylinders.

It is difficult to present the rheology in more details in this chapter, but we invite the interested reader to consult the literature on rheology (see for example [65-67]).

\subsubsection{Surface tension/interfacial tension/ contact angle}

Most of the fluids can be separated in two groups: (i) hydrophilic fluids (fluids having a strong affinity for water. Typical hydrophilic fluids are water solutions, glycerol, alcohol...) and (ii) hydrophobic fluids (fluids which have little or no affinity for water, like oils and most of the polymers). Hydrophilic and hydrophobic liquids in general do not mix together and can form emulsions which are thermodynamically unstable. The hydrophilicity and hydrophobicity of the walls of the microsystems and the interfacial tension of the two fluids are important to know because they will determine if direct emulsions (oil in water) or inverse emulsions (water in oil) are created. It will also influence the stability of the droplets and emulsions formed.

\section{Surface tension}

The physical origin of the liquid/gas surface tension is the attraction existing between neighboring molecules in the liquid state. Molecules located at the liquid/air interface have lost half of their cohesive interaction due to their immediate proximity to the gas interface. Molecules located at the interface are frustrated and therefore will tend to minimize interfacial area.

The surface tension $\gamma$ can be seen as the energy of cohesion of a molecule $U$ divided by its surface $a^{2}$ [68]:

$$
\gamma=\frac{U}{2 a^{2}}\left[J / m^{2}\right]
$$

$U[J]$ is the energy of cohesion, $a[m]$ is the molecule dimension.

Surface tension can also be seen as a capillary force per unit length

$$
\delta W=\gamma d A
$$

In the case of a squared liquid film with one of the side of length $L[\mathrm{~m}]$ the work of the surface tension force is expressed as

$$
\delta W=F d x=2 \gamma L d x
$$

$\delta W[N \times m]$ is the work of the surface tension force, $d A\left[m^{2}\right]$ is the variation of surface, $d x[m]$ is the infinitesimal displacement and $F[N]$ the capillary force.

The surface tension can be measured by different techniques: the spinning drop technique, the Du Noüy ring, the Wilhelmy plate, pendant drop / sessile drop, maximum bubble pressure method to cite the most popular. We will present briefly three of them (i) the Wilhelmy plate method (ii) the rising / pendant drop, and, (iii) the maximum pressure drop. 


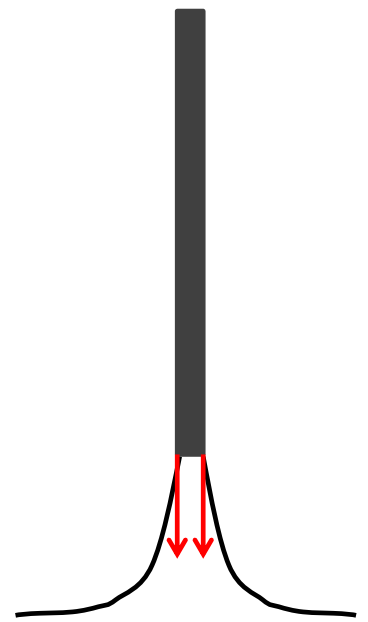

a) Wilhelmy method

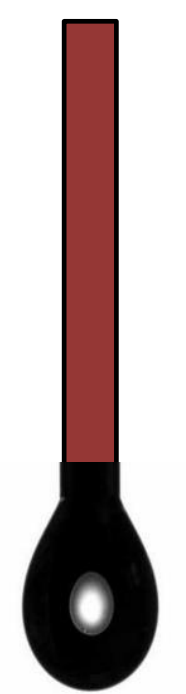

b) Pendant drop method

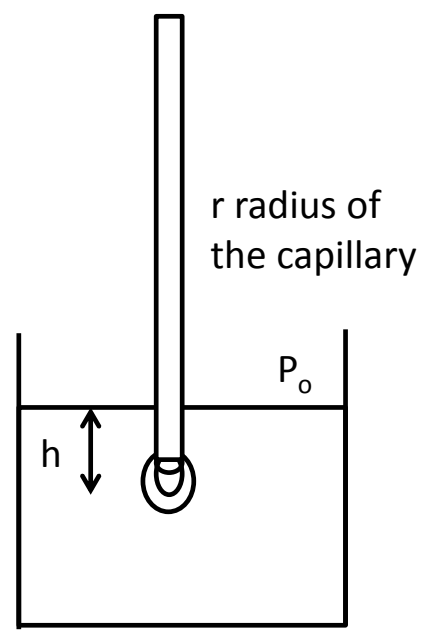

c) Bubble pressure method

Fig. 2.33: Schematic representation of surface tension measurement apparatus

\section{Wilhelmy plate method}

The Wilhemy method is based on the measurement of the capillary force applied on an object. The object is a high surface energy plate, typically in platinum, which is taken to a flame before each measurement to avoid contamination. The plate is slowly pulled out of the bath (see Fig. 2.33a). The force goes through a maximum when the capillary forces are aligned vertically. In that case the force is $F=l \gamma \cos (\theta)$ with an angle $\theta=0$, what becomes $F=l \gamma$.

$I[\mathrm{~m}]$ is the length of the thin plate, $F[\mathrm{~N}]$ is the measured force and $\gamma[\mathrm{N} / \mathrm{m}]$ the surface tension. This method has a precision of about $1 \%$. The Wilhelmy method is very popular due to its simplicity and low cost. The Du Noüy ring method is very close to the Wilhelmy method, but differs essentially by measuring the force on a ring instead of a plate.

\section{Pendant drop and rising drop method}

A bubble or a drop is formed at the tip of a small diameter capillary (see Fig. 2.33b). The equilibrium between the hydrostatic pressure and the Laplace pressure is [68]:

$$
\gamma C=\rho g z
$$

where $\rho\left[\mathrm{kg} / \mathrm{m}^{3}\right]$ is the density, $g\left[\mathrm{~m} / \mathrm{s}^{2}\right]$ the acceleration of gravity, and $z[\mathrm{~m}]$ the vertical coordinate, $\mathrm{C}$ is the curvature assuming an axisymmetric droplet, $C=\frac{-\frac{d^{2} r}{d z^{2}}}{\left(1+\left(\frac{d r}{d z}\right)^{2}\right)^{\frac{3}{2}}}+\frac{1}{r\left(1+\left(\frac{d r}{d z}\right)^{2}\right)^{1 / 2}}$

This method has an accuracy of the order of $1 \%$, and is fully automated by manufacturer like Trackers ${ }^{T M}$ form Teclis Scientific. This method has the advantage to be able to measure surface tension (gas / liquid interface) as well as interfacial tension (liquid / liquid interface).

The maximum bubble pressure method 
The pressure of a bubble forming at a capillary is measured during the growth and ejection. The measured pressure corresponds to (see Fig. 2.33c):

$$
P(R)=P_{0}+\rho g h+\frac{2 \gamma}{R}
$$

Where $P_{0}[\mathrm{~Pa}]$ is the atmospheric pressure, $h[\mathrm{~m}]$ the height of the water, $P(R)[\mathrm{Pa}]$ the pressure measured in the capillary, and $R$ the diameter of the bubble. This pressure goes through a maximum that corresponds to the bubble of the same diameter as the capillary. From that, the previous relation makes it possible to calculate the surface tension.

This method is interesting because it is able to measure the surface tension at short times and its evolution with the time, what is important for the study of surfactant migration or the presence of contamination.

\section{Interfacial tension}

Interfacial tension is the surface tension between two fluids, and it can be measured by pendant/rising drop method presented previously. In this case, the density difference between the two fluids has to be taken into account so that:

$$
\gamma C=\left(\rho_{d}-\rho_{c}\right) g z
$$

Where $C=\frac{-\frac{d^{2} r}{d z^{2}}}{\left(1+\left(\frac{d r}{d z}\right)^{2}\right)^{\frac{3}{2}}}+\frac{1}{r\left(1+\left(\frac{d r}{d z}\right)^{2}\right)^{1 / 2}}$, is the curvature and $\rho_{c}, \rho_{d}\left[\mathrm{~kg} / \mathrm{m}^{3}\right]$ are the densities of the dispersed and continuous fluids respectively.

In the case of two liquid phase microfluidics, the interfacial tension is of primary importance.

\section{Contact angle}

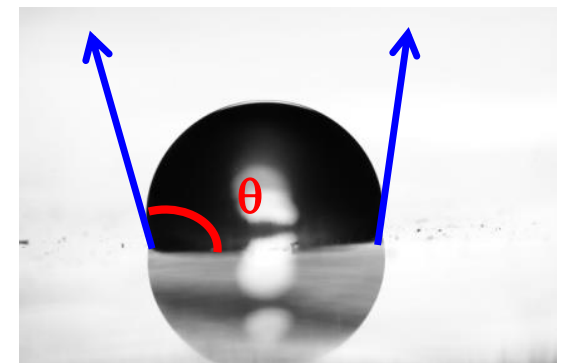

Water droplet on PDMS (hydrophobic surface)

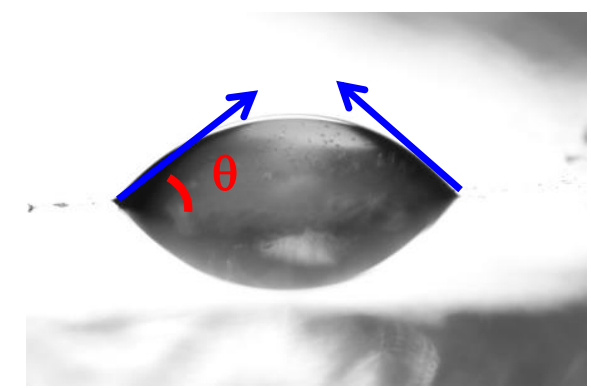

Water droplet on treated PDMS (hydrophilic surface)

Fig. 2.34 Definition of the contact angle between a drop and a surface

The surface of a material is named hydrophilic if the contact angle of a drop of water on its surface is less than $90^{\circ}$, otherwise it is named hydrophobic (see fig. 2.34). It is important to know whether the channel walls are hydrophilic of hydrophilic, since it will determine if droplets of oil in water or droplets of water in oil are formed at the T or flow-focusing junction. In case of hydrophilic walls, oil droplets in water are formed, and inversely in the case of hydrophobic walls, water droplets in oil are formed. As a rule, the stable continuous phase is always the one that wets the surfaces. 
The wetting is named "total" if the droplet spreads completely. It is the case of water on very clean glass or on metal surfaces which are high energy surfaces. If the droplet stays as a drop, the wetting is partial. The spreading parameter is defined as [68]:

$$
\begin{gathered}
S=E_{\text {dry }}^{\text {substrate }}-E_{\text {wet }}^{\text {substrate }} \\
S=\gamma_{S O}-\left(\gamma_{S L}+\gamma\right)
\end{gathered}
$$

Where $E_{d r y}^{\text {substrate }}$ is the surface energy of the dry substrate, $E_{w e t}^{\text {substrate }}$ is the surface energy of the wet substrate, $\gamma_{s o}$ is the surface tension of the solid/air interface, $\gamma_{S L}$ is the solid/liquid surface tension, and $\gamma$ is the liquid/air surface tension.

- If $\mathrm{S}>0$, total wetting, the contact angle $\theta_{E}=0$

- If $\mathrm{S}<0$, partial wetting, and it is considered as rather wetting if $\theta_{E} \leq \pi / 2$ and rather non-wetting if $\theta_{E}>\pi / 2$

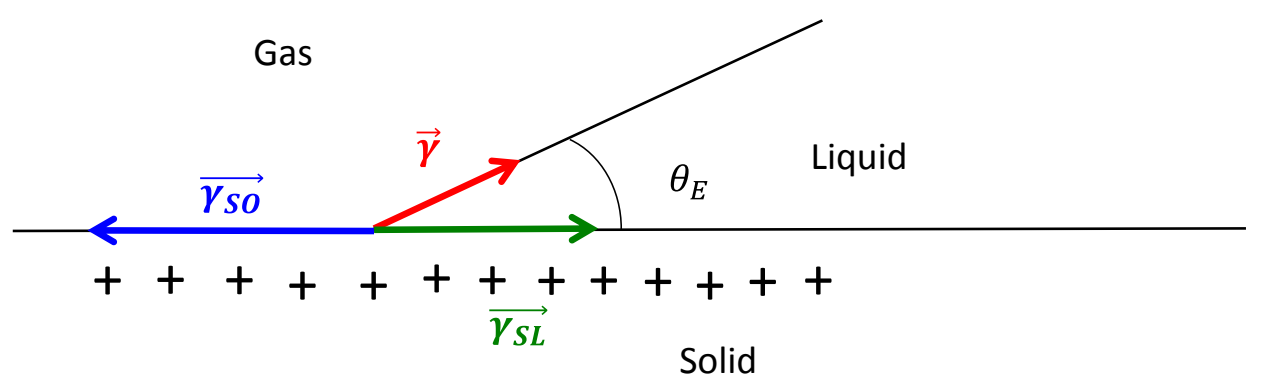

Fig. 2.35: Contact angle and surface tension

- The Young relation is obtained either by a balance of the capillary forces acting on the contact line (Fig.2.35) and projecting them on the solid plan or by calculating the work of the forces for an infinitesimal displacement:

$$
\gamma \cos \theta_{E}=\gamma_{S O}-\gamma_{S L}
$$

Among the different technique existing, we will present three of them which are particularly simple.

- The contact angle $\theta_{E}$ can be measured by shadowgraphy as represented in fig. 2.34 , either directly on droplets of a few microliters (typically about $5 \mu \mathrm{l}$ ) or for more precision by using a software fitting the profile of the droplet and calculating the contact angle.

- For contact angle $\theta_{E}<40^{\circ}$, the mirror droplet technique based on the optical reflectometry is to be favored [68]. The droplet is illuminated by a large laser beam perpendicularly to the substrate, and the reflection cone measured on a screen allows the determination of the mean contact angle with a precision of $0.1^{\circ}$.

- The last technique presented here is the measurement of the contact angle by Reflection Interference Contrast Microscopy. By counting the interference fringes and the number of them, it is possible to calculate the profile of the droplet, and by that, the contact angle with a precision of about $0.1^{\circ}$.

In the case of real, not perfectly clean surfaces, an advancing and receding contact angle can be measured.

Commercial surface tension and contact angle measurement apparatuses are available by manufacturers like Krüss Gmbh, Kibron Inc, Dyne Testing Ltd, USA KINO Industry CO. Ltd, Biolin Scientific or Data Physics Intruments, $\mathrm{GmbH}$, Germany, among others. 


\section{Surfactant}
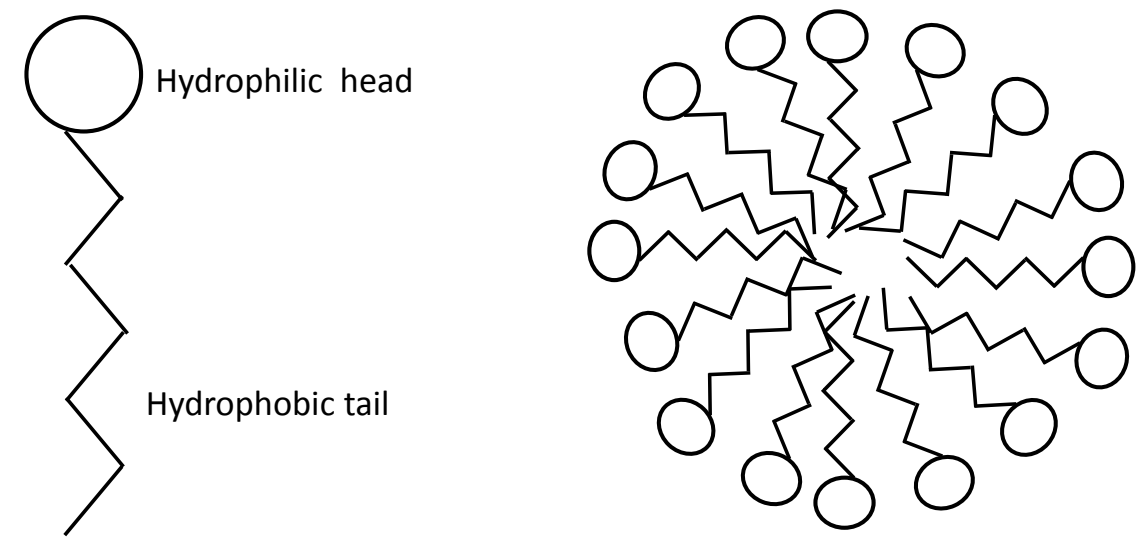

micelle

Fig.2.36 Schematic representation of a surfactant molecule and micelle formed by molecules of surfactants

Surfactants are usually added to the oil / water mixture in order ease the emulsification process and to stabilize the emulsion. Surfactant are molecules that own a hydrophilic group (an anionic, cationic or nonionic group named "head"), and a hydrophobic group (usually a hydrocarbon chain named "tail"). Because of their hydrophilic head and hydrophobic tail, they are prone to locate themselves at the interfaces. They are used at concentrations going from a fraction of a percent to a few percent. They modify the surface tension and stabilize the droplet formed. The Critical Micelle Concentration (CMC) is the concentration above which micelles i.e. aggregates of surfactant molecules are formed (see Fig. 2.36). It is important to be beyond the $\mathrm{CMC}$ to avoid interfacial tension variations by depletion, what may occur during the droplet formation process. The CMC can be determined experimentally in measuring the surface tension for different concentrations of surfactant by one of the methods presented earlier. Below the CMC, the surface tension changes strongly with the concentration of surfactant while it remains almost constant above the $\mathrm{CMC}$.

The previously presented physical properties are useful to calculate the Capillary number, i.e. the relative effect of the viscous force to the interfacial forces:

$$
C a=\frac{\frac{\mu U}{r}}{\frac{\sigma}{r}}=\frac{\mu U}{\sigma}
$$

Where $\mu[\mathrm{Pa} . \mathrm{s}]$ is the viscosity, $U[\mathrm{~m} / \mathrm{s}]$ the velocity, and $\sigma[\mathrm{N} / \mathrm{m}]$ the interfacial tension.

The capillary number is the driving parameter of most of the two phase microfluidics systems, and the most suitable non-dimensional number characterizing regimes of formation of droplets or bubbles.

\subsubsection{Experimental aspect of microfluidics}

\subsubsection{1 $\mu$ PIV experimental set-up}

$\mu$ PIV has been developed on inverted microscopes [69]. Numerous companies are building these kinds of microscopes: Zeiss, Leica, Olympus, Nikon.... The easy optical access of the microfluidic microsystems (usually in transparent materials like Glass, PDMS, Plexiglass or other transparent plastics), has been used to visualize the flow field on a plan. A typical set-up is represented schematically in Fig. 2.37. 


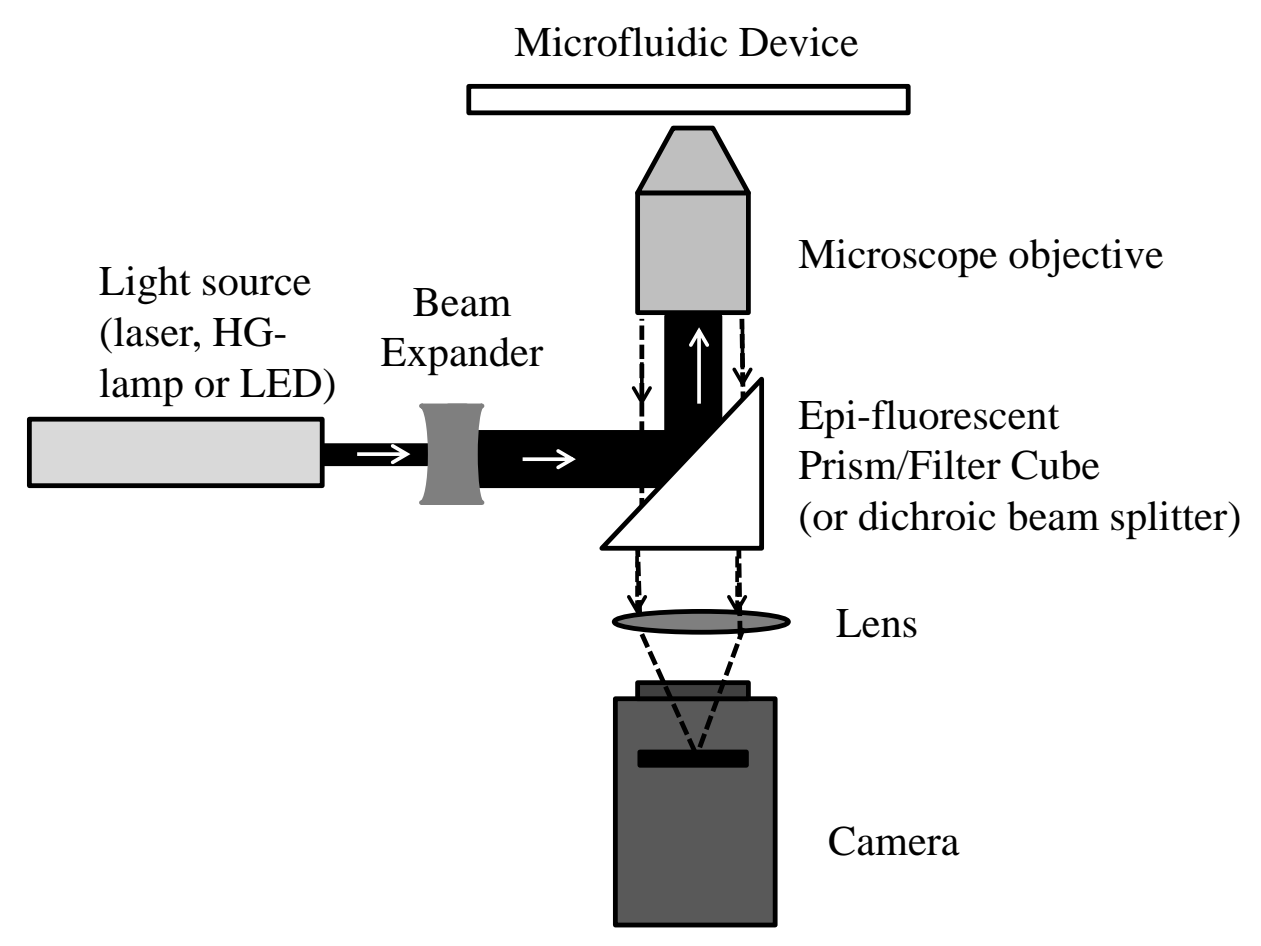

Fig. 2.37: Schematic representation of a $\mu$ PIV set-up (see [69, 70] and [57])

A strong light, usually a double-pulsed laser light (typically a Yag Laser), is used to illuminate fluorescent particles. But other light sources can be used like mercury lamp [55] or strong LED [72]. In the latter case, shadow imaging of non-fluorescent particles are used instead of fluorescent particles. In case of a laser, an epi-fluorescent prism transmits the laser light to the microfluidic microchannel through the objective lens of the microscope. The fluorescent particles absorb the laser light and re-emit light in a different wavelength. This fluorescent light crosses the objective lens and the epi-fluorescent prism to be received on the camera. The epi-fluorescent prism includes a filter that cuts the light at the wavelength of the laser and lets the fluorescent light go through in order to get rid of incoming laser light, of the scattered light, of the multiple reflections, of the background illumination inhomogeneity [69] and of the speckle patterns. Images are commonly taken on 12 bits CCD or CMOS camera. Double image cameras are chosen when double-pulsed laser are used.

\section{Camera}

Microfluidic experimentalists often rely on high speed cameras for imaging and high temporal resolution $\mu$ PIV. The main brands of high speed cameras are Phantom, Photron and Optronis. They are useful for two reasons: (i) Displacements on images are large because of extensive zooming on microscopes. Therefore, short exposure times and high frequency imaging are necessary to capture temporal evolution of the velocity field or the movement or deformation of droplets or bubbles, (ii) The physical limit of light illumination on the microchannel must be compensated by a high sensitivity of the high speed cameras in order to freeze images (very low exposure times are necessary to avoid blurred images).

Exposure times as low as $1 \mu$ s and frequencies as high as tens of thousands of images per second are used routinely in microfluidics. Since microsystems are transparent, imaging of droplets by back illumination and image analysis on softwares like ImagJ or Matlab have been a major tool to estimate the size of droplets, mixing or droplet formation to cite a few.

\section{Depth of field}

Unlike the traditional PIV where the measurement plane and its thickness is defined by the thickness of the illuminating laser sheet, in the case of $\mu$ PIV the illumination is a volume illumination, and the thickness of 
the measurement plan is defined by the depth of field [69] or depth of focus [71] of the optical system. The question on how to define the thickness of depth of field form the optical system has appeared very early in the development of $\mu$ PIV systems [69-71].

The estimated depth of field $\delta_{\mathrm{Zm}}$ of the microscope objective has been estimated by [69] to be :

$$
\delta_{Z m}=\frac{3 n \lambda_{0}}{N A^{2}}+\frac{2.16 d_{p}}{\tan \theta}+d_{p}
$$

Where the different parameters are : $N A$, numerical aperture of the microscope objective, $d_{p}$, the particle diameter, $\lambda_{0}$ wavelength of the light, $n$ index of refraction and $\theta$ the half angle of the objective ( $N A=$ $n \sin \theta)$.

To compare the previous formula with experimental results (see Fig. 2.38), $1.09 \mu \mathrm{m}$ particles have been placed between two glass plates and images have been taken at different objective-microsystem distances (see Fig. 2.38). The experimental measurements are in good agreement with the $\delta_{\mathrm{zm}}=16.5 \mu \mathrm{m}$ depth of field of the microscope calculated by the above analytical expression [69].
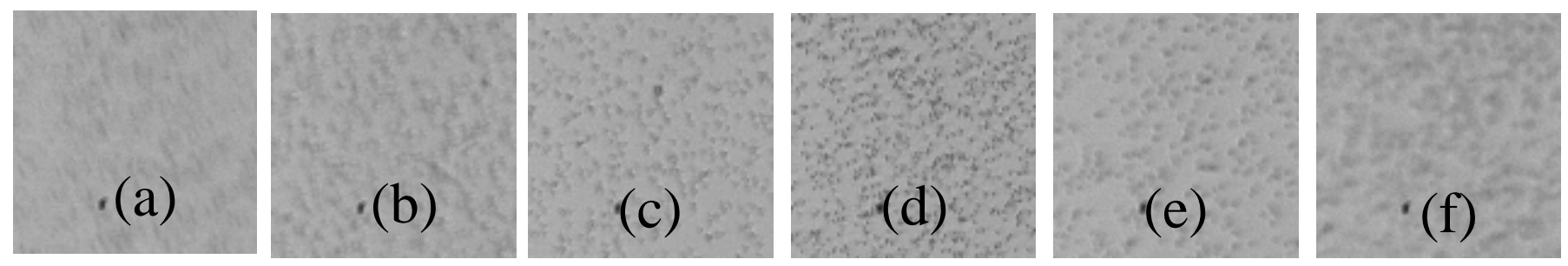

Fig. 2.38. Image of $d_{p}=1.09 \mu \mathrm{m}$ diameter particle measured at different distance from the focal plane a) 40 $\mu \mathrm{m}$ below the focal plane, b) $20 \mu \mathrm{m}$ below, c) $10 \mu \mathrm{m}$ below, d) at the focal plan, e) $10 \mu \mathrm{m}$ above, f) $20 \mu \mathrm{m}$ above, the objective lens is $\times 20$, the numerical aperture is 0.40 (image extracted from Carrier \& al. 2015 [72]).

Olsen \& Adrian [71] defined a depth of correlation as the depth over which particles significantly contribute to the correlation function. They give the analytical formula

$$
Z_{\text {corr }}=\left[\frac{(1-\sqrt{\varepsilon})}{\sqrt{\varepsilon}}\left(f^{2} d_{p}{ }^{2}+\frac{5.95(M+1)^{2} \lambda^{2} f^{4}}{M^{2}}\right)\right]^{1 / 2}
$$

Where $d_{p}$ is the particle diameter, $M$ the magnification, $\lambda$ the wavelength of the light emitted by the particle, $f$ is the focal number of the lens, $f \approx n_{0} /(2 N A), N A$ being the numerical aperture and $n_{0}$ the index of refraction of the immersion medium. $\varepsilon$ is the ratio above which the weighting function contribution of a particle located beyond $Z_{\text {corr }}$ is negligible. In practice, $\varepsilon$ is usually taken as 0.01 when assuming that the outof-focus particles have an intensity of $1 \%$ of the in focus particles [57]. Bourdon \& al. 2004 [70] have validated experimentally and computationally the accuracy of this analytical formula.

\section{Particles}

Particles used as seeding particles in $\mu$ PIV must follow the flow field, therefore (i) they must be well dispersed in the fluid (particles may be treated by surfactant grafting or by adding small amount of surfactant in case of polymer particles in a hydrophilic fluid), (ii) they must have a density close to the density of the fluid to avoid sedimentation or creaming effects, (iii) they must be small enough to follow the flow, large enough to avoid large Brownian motion that would add scattering to the velocity measurement, and large enough to be visible on several pixels of the camera. Numerous companies are providing calibrated particle, among them www.microparticles.de, Bangs Laboratory (www.bangslabs.com), www.sigmaaldrich.com, Merck-Millipore, Harvard Office of Technology Development, Duke Scientific (www.dukescientific.com), Invitrogen (www.thermofisher.com), Molecular Probes ${ }^{\mathrm{TM}}$ and the companies 
selling $\mu$ PIV equipment. A general tradeoff is to use seeding particles of the order of $1 \mu \mathrm{m}$. In case of fluorescent seeding, particles can be smaller because not the particles, but the emitted fluorescent light is imaged.

\section{Cross correlation}

The seeded flow is illuminated by a laser pulse of a few picoseconds while the first image is taken. The second image is taken during the burst of the second pulse (see Fig. 2.39). Each image is divided into interrogation areas of $16 \times 16$ or $32 \times 32$ or $64 \times 64$ pixels or combination thereof. It is recommended to have around 10 particles per interrogational areas. The most common technique for calculating the flow field is to use a pixel by pixel cross correlation between corresponding interrogational areas.

$$
C(s)=\iint_{\text {Area }} I_{1}(X) I_{2}(X-s) d X
$$

The correlation produces a peak identifying the most common particle displacement $d X$ (see Fig. 2.39). A velocity map is obtained by repeating the cross correlation over each interrogational area.

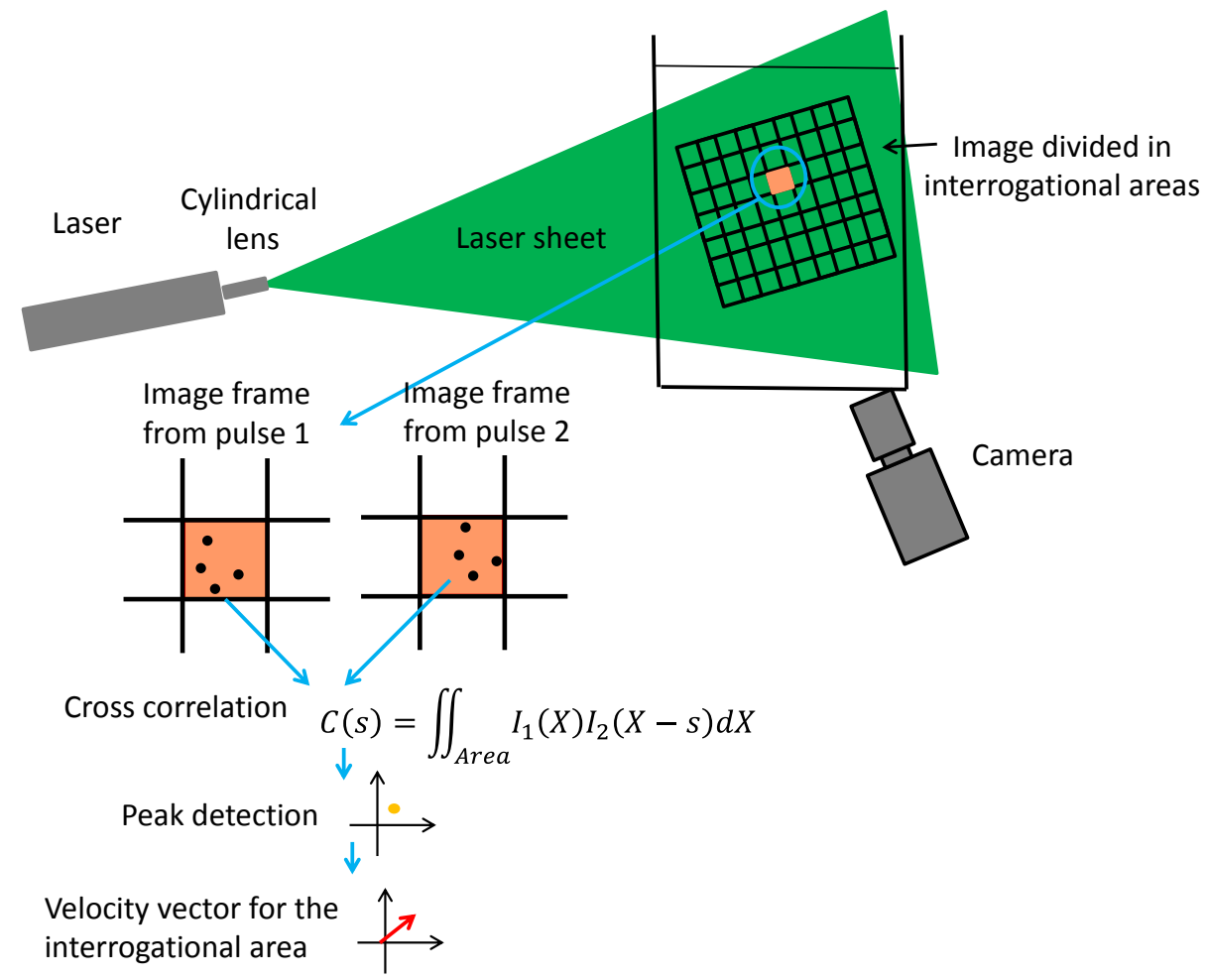

Fig. 2.39: Particle Image Velocimetry set-up and principle of the flow field measurement, i.e. Cross correlation between corresponding interrogational areas

Flow field can also been obtained by Particle Tracking, but in this case the working conditions are more severe like having less seeding particles and a good contrast so that particle can be identified clearly.

\section{Brownian motion}

At small scale, Brownian motion may play a major role. Calculation of the impact of Brownian motion on the velocity has been first studied by Santiago et al. 1998 [55]. For a time interval of $\Delta t$ corresponding to the time interval between the two images used for the PIV, the mean square distance of diffusion is $\left\langle s^{2}\right\rangle=2 D \Delta t$, where

$$
D=\frac{k T}{3 \pi \mu d_{p}}
$$


Where $d_{p}$ is the particle diameter, $k$ the Boltzmann's constant, $T$ the temperature and $\mu$ the dynamic viscosity of the fluid. The displacement of a particle following a steady flow at a velocity $u$ is $\Delta x=u \Delta t$ over a time interval $\Delta t$.

Therefore, the relative error due to Brownian motion is estimated by:

$$
\varepsilon_{B}=\frac{\left\langle s^{2}\right\rangle^{1 / 2}}{\Delta x}=\frac{1}{u} \sqrt{\frac{2 D}{\Delta t}}
$$

$\varepsilon_{B}$ has to remain low.

Devasenathipathy et al. 2003 [73] notice that the diffusive uncertainty decreases as $1 / \sqrt{N}$, where $\mathrm{N}$ is the number of particles per interrogational area.

Most of the $\mu$ PIV are 2D, but 3D $\mu$ PIV and tomographic have recently been developed. The reader interested should consult the recent reviews of Cierpa and Kähler 2012 [57] on these 3D $\mu$ PIV.

\subsubsection{Experimental field where $\mu$ PIV has been a major measurement technique}

\subsubsection{Droplet formation}

Droplet manipulation is one of the most successful applications of microfluidics (see fig. 2.40). The reproducible formation of droplet serves various applications such as high throughput screening [20]. Our understanding of the droplet formation in microsystems has been significantly improved using the $\mu$ PIV measurement technique (see [72] and [74, 75]).
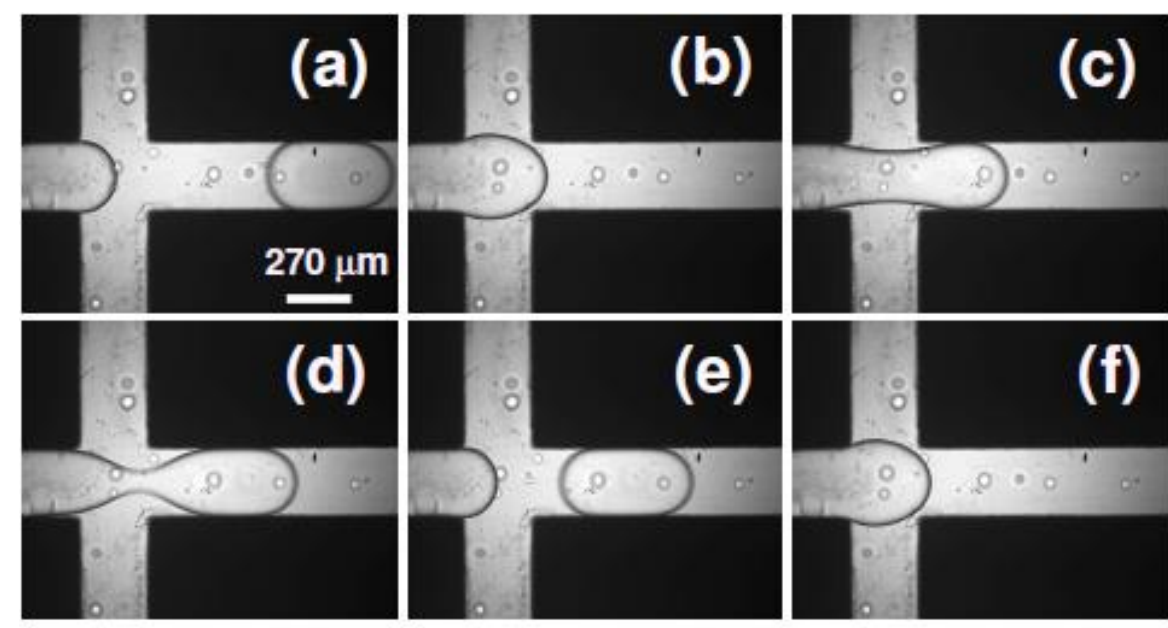

Fig. 2.40: Droplet formation in microsystems (image extracted from Funfschilling et al. 2009 [75]).

$\mu$ PIV allows the measurement of the stress on an interface. Knowing the viscosity, the local velocity gradient $\gamma$ can be calculated from the flow field, and the local shear stress on the droplet interface (or cell membrane in the case of a cell) can be calculated by $\tau=\mu \dot{\gamma}$ (see Fig. 2.41). 


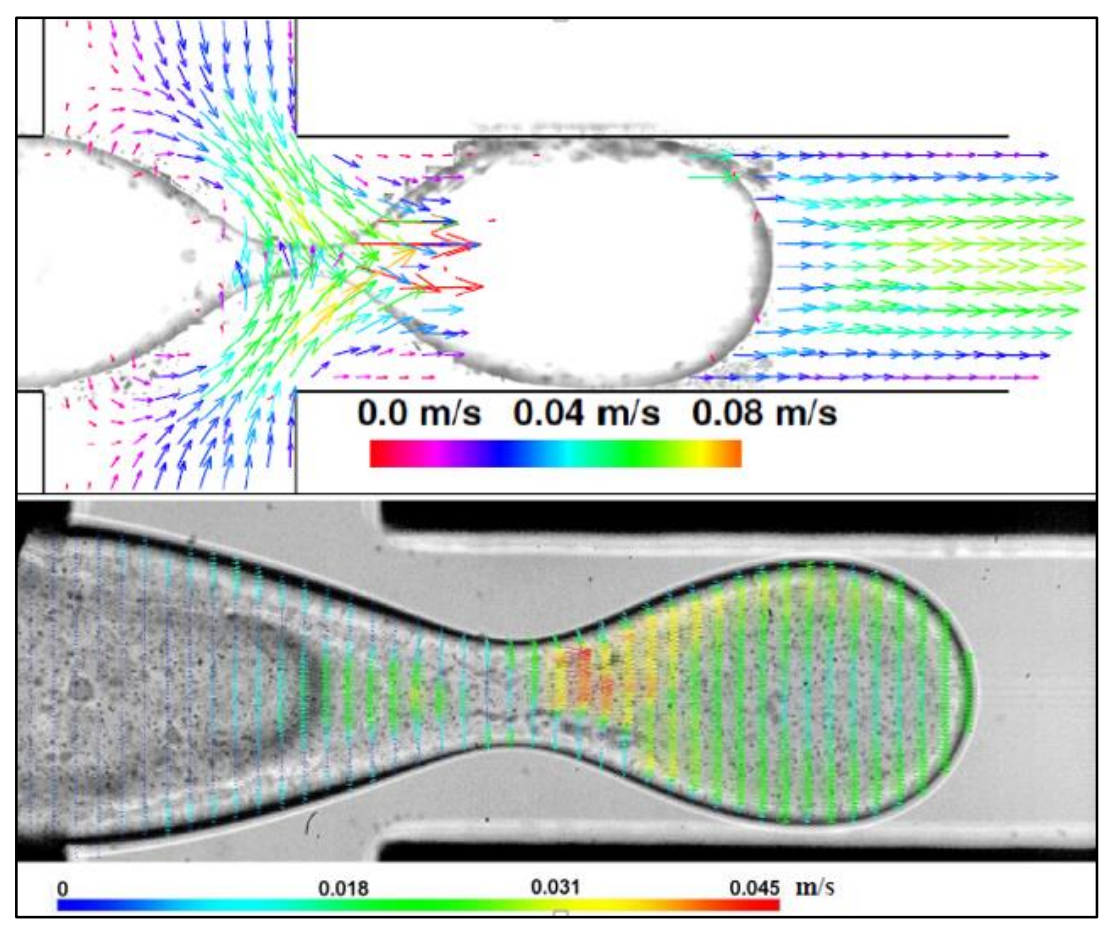

Fig. 2.41: $\mu$ PIV measured flow field inside and outside a droplet in formation. The step of the neck thinning on the way to rupture is represented. The physical mechanism of the formation of droplet is well depicted

by the flow field measurement (image extracted from Funfschilling et al. [75] and Carrier \& al. [72]).

Elongational flows are also measured by this technique (see Fig. 2.42). It is important information that enables the measurement of mechanical properties of membranes of cells, or the elasticity of DNA [76].

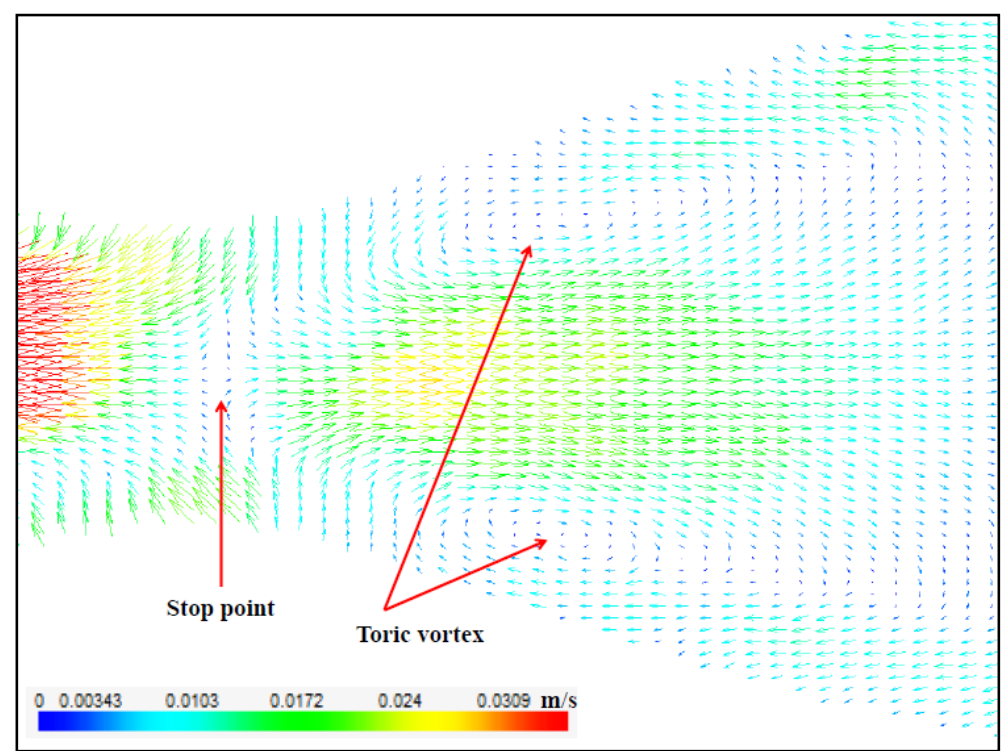

Fig. 2.42: Flow field in a droplet in formation. A stop point is clearly visible between two toroidal vortices respectively in the droplet to be detached and in the finger on the way to retreat (image extracted from Carrier \& al. [72]).

\subsubsection{Stress effect of blood flow on growing endothelial cell on the arterial wall}

Abdominal Aortic Aneurism and Brain Aneurism are still difficult to prevent and to cure. Recent research pointed out the role of the endothelial cells and the wall shear stress to which they are exposed on the development of Aneurism [77]. Furthermore, shear-induced shear stress modulates the gene expression of 
endothelial cells [78]. $\mu$ PIV in microchannel is the way (and probably the only reliable way) to study the behavior of endothelial cells under the periodic cardiac blood flow that induces a periodic shear stress on the arterial wall covered with endothelial cells. Micropumps reproducing the cardiac cycle can be used to control the flow in a Hele-Shaw cell (a thin cell with wide $X$ and $Y$ dimensions, so that the flow can be considered as nearly 2D), where the walls are covered with endothelial cells [62]. As shown in Fig. 2.43 in the case of a droplet in formation, the wall shear stress can be extracted from the flow field measured by $\mu \mathrm{PIV}$ by multiplying the near wall velocity gradient by the viscosity of the fluid $\tau=\mu \gamma^{\prime}$.

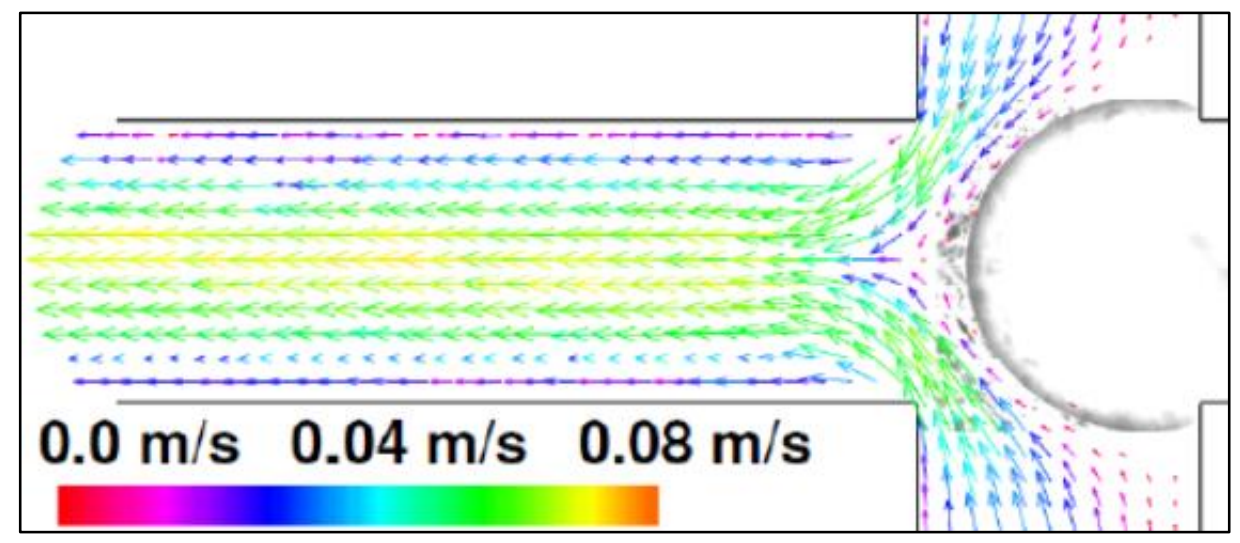

Fig. 2.43: Flow field around a droplet in formation. The shear stress applied locally on the droplet surface $\tau=\mu \gamma$ can be measured knowing the viscosity of the fluid and the shear rate $\gamma$ calculated from the flow field (image extracted from Funfschilling et al. [75]).

Rossi et al. [62] have determined the topography and shear stress distribution over individual cells with subcellular resolution by the $\mu$ PIV technique. In vitro experiments on endothelial cells in microsystems have the advantage of better established and more reproducible flow conditions and are less subject to biological variations. The study of Rossi et al. [62] reveals that the shear responsive gene KLF2 (Krüppel-like factor-2) increases its activity as the shear stress level increases.

\subsubsection{Wall shear stress measurements in the arterial system of an embryonic chicken}

Wall shear stress is strongly involved in the development of the circulatory system. In the embryonic stage, blood flow affects the development of the primitive heart and blood vessels [79]. The alteration of the flow in the heart leads to malformations in the cardiovascular system [79]. The biological response to the haemodynamic forces can be short term for the control of vascular tone or long term through gene expression [25]. It plays also a role in the etiology of a number of diseases, for example regions with low or oscillating wall shear stress have been linked to atherosclerosis [80]. The blood flow and wall shear stress have been measured in vivo in the outflow of an embryonic chicken heart $[25,26]$ by the $\mu$ PIV technic with large temporal resolution. $\mu$ PIV is a very reliable non-intrusive method for measuring the velocity profile with a sufficient spatial resolution to calculate the wall shear stress from the closed wall velocity gradient and therefore enables the study of the interaction between hemodynamics and the surrounding tissue [80].

\subsubsection{Rheology}

According to [63], $\mu$ PIV in micro channels offers the possibility to develop rheometers suitable for probing rheological properties of weakly elastic fluids that appear as Newtonian under the conditions attained in a conventional rheometer. Sugii et al. [81] have measured the rheology of blood flow in microcirculation invitro by measuring both red blood cell velocity and plasma velocity with a high-speed $\mu$ PIV.

\subsection{Main ex-situ characterization techniques for bio-devices (Norbert Dumas, Wilfried Uhring)}




\subsubsection{Introduction}

Microfluidics devices are preferably made of see-through material enabling the characterization of the biochemical reaction inside the chip mean, besides the possibility of visual inspections and flow characterization. This chapter focusses on main optical techniques that do not require integrated part inside the chip, except solutions that are injected inside the canal (e.g. fluorophores). There are other physical principles that would be classified as not requiring integrated part but they are not treated here (radioactive marker, NMR...) are less common. Some optical setups are presented in details so that one can make it from detached optical element and an optical table. This goal is also to explain role of each element. Alternatively, inverted microscope with some elements already assembled can be bought directly from a manufacturer. Microscopes are convenient in a biological lab environment because it offers the binocular visualization. Optical readers for microfluidics are also commercially available.

\subsubsection{Colorimetry}

\subsubsection{Measurement principle}

Colorimetry is a well-established method for biological assay. The colored product can be the result of a reaction. For example, the Berthelot reactant produces a blue-green solution in a quantity depending on the ammonium ion concentration. It can also be used identify a sample solution it from its color. This is named barcoding.

The setup is based on the measurement of the absorbance of the sample, which is considered to be in a droplet. The basic Beer-Lambert law is used to characterize the absorption for a monochromatic light source propagating in a homogeneous and isotropic medium and can be expressed as:

$$
I=I_{0} \cdot 10^{-\varepsilon_{\lambda} l . C}
$$

Or

$$
A_{\lambda}=-\log _{10} \frac{I}{I_{0}}=\varepsilon_{\lambda} . l \cdot \mathrm{C}
$$

Where $\mathrm{I} / \mathrm{I}_{0}$ is the transmittance of the medium, $A$ is the absorbance, also called the optical density, that are both unitless, $\varepsilon_{\lambda}$ is molar extinction generally expressed in $\mathrm{L}_{\mathrm{mol}} \mathrm{m}^{-1} \cdot \mathrm{cm}^{-1}, \mathrm{I}$ is the length of the optical path in the medium and $C$ in the molar concentration generally express in mol. $\mathrm{L}^{-1}$.

The molar extinction $\varepsilon_{\lambda}$ is related to the chemical species, the wavelength and the temperature. Considering a microfluidic canal of thickness $l$, if $\varepsilon_{\lambda}$ is known, the concentration of the chemical species can be assessed by measuring the absorbance.

In presence of several chemical compounds the absorbance of each species are added so that the total absorbance of a droplet is given by:

$$
A_{\lambda}=\sum_{i=1}^{n} A\left(\varepsilon_{\lambda i} l . \mathrm{C}_{i}\right)
$$

As a consequence, $n$ different concentrations of chemical species can be discriminated by using $n$ different wavelengths (or bands) and by solving the $n$ resulting equations. Another method consists in varying the species concentrations within the sample while the wavelength is constant. For these measurements, the reference intensity $I_{0}$ has to be measured. The simplest method is to ensure that the targeted compound concentration is almost null in a specific sample. If the carrier fluid, the other species concentration and the reflective index don't change, $I_{0}$ is the intensity measured with this reference droplet(s). For paper based 
microfluidics, it can be difficult because a dry paper has a different reflective index than wet paper [Aud2009].

The scheme of the general principle optical setup is given in figure 2.44. Reflective based methods are possible but less sensitive. This is a very straight forward measurement approach and many parts of this setup can be very simple and cheap. The main challenges of this setup are:

1) Ensuring that most of the light is passing through the sample, e.g. a droplet, in order to obtain the maximal sensitivity and to be able to carry out a quantitative assessment of the measured concentration.

2) Ensuring a low noise measurement in order to obtain a sufficient signal to noise ratio. Indeed, in regards with the very low canal thickness $l$, the variation of the light intensity can be very weak, thus the signal to noise ratio must be kept high in order to be able to discriminate the lowest concentrations.

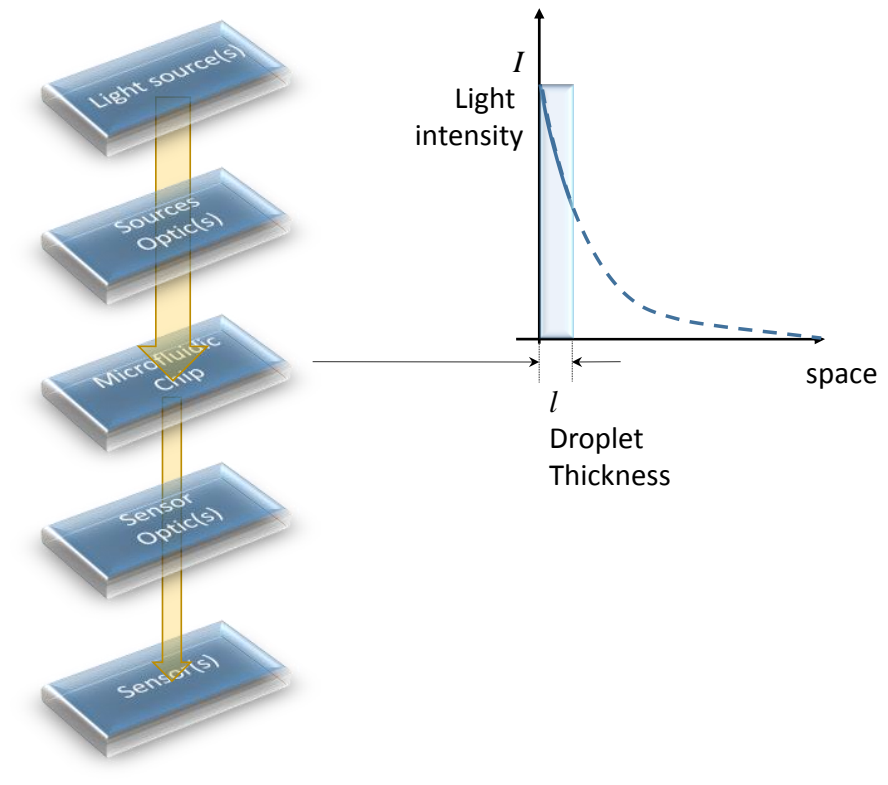

Fig. 2.44 General principle of a colorimetry optical setup

\subsubsection{Implementation}

Quantitative colorimetry requires a monochromatic measurement, thus monochromatic LED or laser diode are very suitable as a light source for this application [82]. Indeed, the commercially available LEDs are covering a large spectrum from UV to visible and Near infrared. Alternatively, polychromatic light sources such as white LED, incandescence/halogen lamp [83] and supercontinuum can also be used in addition with optical bandpass filter. For instance, thanks to its additional Bayer filter that acts as a bandpass filter, a simple mobile phone camera can be a cost effective devices for colorimetric device [84, 85]. Because it is cost effective, colorimetry is often used with very low cost paper-based microfluidics. For example, the use of a switched tri-color LED (Red, Green Blue) and a single photodetector allows transmittance colorimetry measurement with very low cost [86] ( 50\$).

However for a complete characterization, a wavelength sweep is required. It can be implemented with a white light source followed by a prism or a monochromator. A moving hole determines the wavelength. For example, it has been used to characterize more precisely a low cost colorimetric system [86]. Improvement of the technique includes modulating the light source and using a lock-in amplifier for readout. Placing the light source and the sensor in the canal axis is better, as the thickness is increased, but it requires chip 
modification. Sometimes the reference sample with no product is measured in separate chamber to that the analysis chamber is used only once.

\subsubsection{Fluorescence spectroscopy}

\subsubsection{Principle of fluorescence spectroscopy}

Fluorescence spectroscopy is based on the absorption of light by a fluorescent substance which will re-emit a photon at higher wavelength [87]. This Stockes shift is exploited to separate the fluorescence light from the source (see Fig. 2.45). The fluorescent substance is called fluorophore or fluorochrome. It can either be the biomolecule we want to measure directly (intrinsic), or a fluorophore attached to a target molecule (extrinsic). A fluorophore can also be used as an indicator with fluorescence properties which depends on the presence of the target molecule. The progress in biochemistry provides many ways (from simple and direct to complex and indirect) to extract the information of interest for the biologist from a measurement of fluorescence. Once the biochemistry is set, three main techniques are applicable for Lab on Chip to read the fluorescence information: Intensity, Polarization and Lifetime. It is important to maximize the intensity of light that is measured for the three techniques because of noise and other parasitic signals. It is based on the principles described in this section that are also applicable to phosphorescence, which is another type of luminescence.

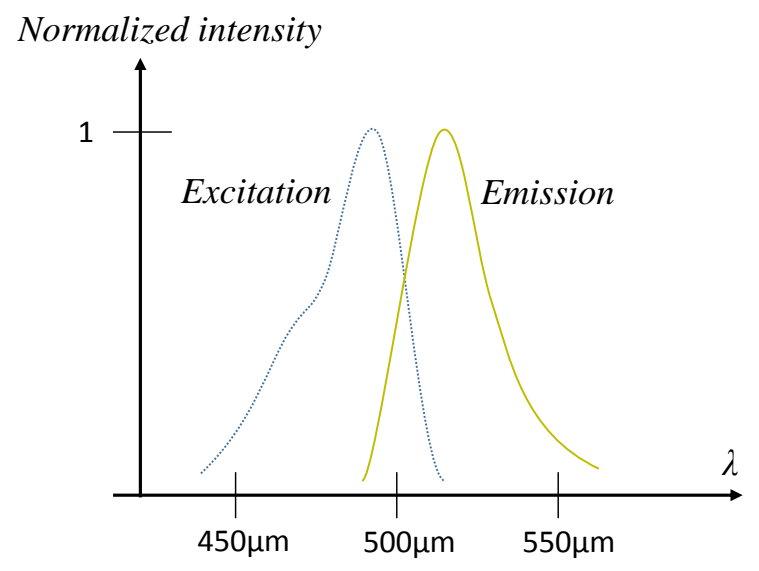

Fig. 2.45 : Image of a fluorescence excitation and emission spectrum (fluorescein)

Fluorescence measurement offers a better contrast than colorimetry. For example let's consider that one wants to make the difference between no product and a product at low concentration. Colorimetry measures a small decrease of a large light intensity caused by absorption. As a consequence, the relative variation is weak. Although fluorescence depends also on absorption of photons, fluorescence is detected only if there is product because what is detected is only what is reemitted. Therefore the relative variation is much larger. This is important because, even for high throughput application, concentrations are often lower than $1 \mu \mathrm{M}$. The detection limit of fluorescence spectroscopy can be lower than $1 \mathrm{nM}$. In this respect, colorimetry is very limited as the detection limit is in the order of $1 \mu \mathrm{M}$.

Nonetheless it should be noted that, because this technique still depends on absorption, the fluorescent signal intensity increases with the effective number of fluorophore molecules that has actually absorbed light. In order to not waste the incident light, the light intensity can be concentrated using a collimated laser beam as a light source. However the relative shallowness of the microfluidics canal is a problem because for a typical concentration of product the laser beam is not totally absorbed and cross the canal. In this respect, techniques depending only on the detection of product at surface level (i.e. CHEMFET, SPR) do not suffer from the thickness reduction. However these techniques require the integration of a sensing element inside the canal contrary to the fluorescence spectroscopy. 
The product absorbs the following light intensity $l_{\mathrm{a}}$ according to the beer lambert law:

$$
I_{a}=I_{0}\left(1-10^{-\varepsilon_{\lambda} \cdot l \cdot C}\right) \sim I_{0} \cdot \varepsilon_{\lambda} \cdot l \cdot C \cdot \ln (10)
$$

Where $I_{0}$ is the incident light intensity from the source. If the laser spot is smaller than the canal and we neglect the loss on the light path from the laser to the inside of the canal, $I_{0}$ is the laser intensity. $\varepsilon_{\lambda}$ is the molar extinction coefficient of the product and is dependent of the wavelength of excitation. $I$ is the thickness for a light coming orthogonally to the plane of the microfluidics chip. $\mathrm{C}$ is the concentration of the product. For low concentration and thickness, the absorbed light is proportional all these four parameters $\left(I_{0}, \varepsilon_{\lambda}, I, C\right)$.

The fluorescence emission depends on $I_{a}$ but also on the quantum yield (Q.Y.) because not all absorbed photon are reemitted. For the fluorescein fluorophore this quantum yield is $95 \%$ in a 0.1 solution of $\mathrm{NaOH}$ at $22^{\circ} \mathrm{C}$ but for Tryptophan, which is one of the amino acid responsible for the fluorescence of many proteins, the quantum yield is only $14 \%$ in water at $20^{\circ} \mathrm{C}$. This quantum yield depends on the non-radiative decay, quenching by other species (e.g. dissolved oxygen) and transfer of an exited state such as RET (resonance energy transfer). The latter can induced the emission of a photon but not from the considered fluorophore. When excited with a constant light source, the average reemitted intensity is $I_{e}=k Q l_{a}$, where $\mathrm{k}$ is a coefficient taking into account the Stockes shift on the entire emission spectrum. It is smaller than 1 because the emitted photons are less energetic than the absorbed ones. As this light is reemitted in all directions, only a fraction of it can be collected through a microscope objective.

The absorption of a photon is quasi instantaneous as soon as the photon arrives but it is not reemitted immediately. The average time to reemit the photon is called the lifetime $(\tau)$. The probability that the photon is emitted after a time $t$ is:

$$
p(t)=\frac{1}{\tau} e^{-\frac{t}{\tau}}
$$

To observe this phenomenon, a very short pulse of light should excite the fluorophores and a high bandwidth light sensor should measure the temporal response that is an exponential decay. When the incident light is constant the emitted light is also constant because of averaging effects. Zooming in time and looking at the emission of photons one by one show that it is a Poisson process. This results in quantum noise that can affect the measure for low intensity [88].

If the light is polarized, only the fluorophores with a transition moment aligned with the electrical field of the incident light are excited. The polarization of fluorescence will depend on the orientation of the transition moment at the emission of the photon. Due to agitation the fluorophores move and rotate and one can define a rotational correlation times that depends strongly on the size of the fluorophore. It is typically in the order of ns or tens of ns for a macromolecule. If the lifetime is much higher than this time, the polarization of the light is lost. As the lifetime of phosphorescent molecules is in the order of ms, anisotropy cannot be applied in this case. Fortunately the lifetime of many fluorophores are typically in the order of ns or tens of ns. Therefore one can expect a change in the polarization of light if the fluorophore attaches to a macromolecule bigger than itself.

\subsubsection{Considerations on the setup for Lab On Chips}

Research and development in biology make intensive use of spectrofluorometers, whether the sample is placed in a chamber or in microplate array for parallelization and achieving high throughput analysis. However, although the same principle is used and the optical setup is similar for analysis, most of the time it has to be adapted to read a microfluidics chips and the optical setup is built around the chip. Sometimes the chip is placed on an inverted epifluorescence microscope, offering great flexibility for visualization. Obviously the format of the microfluidics chips differs but also the way of achieving high throughput and small volumes is a completely different concept. The microplate readers use parallelization of the sensors 
and robotic arms while the samples are static in a well. Using microfluidics, the sample is preferred to be contained in a moving microdroplet although sensors can be easily parallelized due to small size. With a rate of 1000 droplets/s the same rate of analysis is achieved with a single sensor. This is much faster than using robotic arms while simplifying the setup.

Here, we will consider mainly that samples are contained in a flow of droplets passing in a canal, unless specified otherwise. Static analysis is possible with the same apparatus by moving the chip (with either a manual or a motorized system). One can also analyze static droplets in stop-flow or EWOD (electrowetting on dielectric) or also Dielectrowetting [89]. Differential measurement with moving droplets is easy because, when the oil separating two droplets is passing by, a reference signal can be read if the oil is non fluorescent. Ideally the spectrofluorometer can excite fluorescence and read it at any wavelength. It uses a white and stable light source (e.g. a Xenon lamp) that is either filtered or passed through a monochromator in order to select a wavelength. While it can still be used, it is more convenient to use a collimated laser source. Whereas the wavelength is fixed, the size of the beam offers an easy way to focus the intensity for more efficiency. If several wavelength of excitation are required, lasers beam are combined as well as the detectors for each spectrum of emission. Laser can also be coupled to an optical fiber to excite the fluorophore. The emission can also be collected with the optical fiber so that the probe location can be easily displaced.

A limitation of the laser beam intensity is the alteration of the microfluidic chips or the samples. At high intensity photobleaching of the fluorophore can be problematic for long measurement. Material should be chosen to be transparent to the wavelengths of interest, which are not necessary in the visible spectrum. For example, common borosilicate glass absorbs UV below $300 \mathrm{~nm}$. The fluorescence of the material should be low and is problematic for weak intensity measurement. The fluorescence of the glass depends strongly on impurities inside. In [89], the experiments at 4 wavelengths show that a commercial borosilicate glass has a slightly lower autofluorescence than plastic materials (PDMS, PC, COC and PMMA). It also depends on the excitation wavelength. Photobleaching of the material is possible but it does partially recover [90].

A confocal setup also helps to remove the fluorescence of the material and other interfering lights by reducing the depth of focus. This way, only the light coming from the focal plane is collected. As only what happen in the thickness of the canal is of interest, the optical requirements are relatively low, i.e. much less than a confocal microscope with 500nm or less axial resolution. In [91], authors use a relatively big pinhole size of $500 \mu \mathrm{m}$ and a $20 \times 0.75 \mathrm{NA}$ objective. It results in a $14 \mu \mathrm{m}$ probe depth, still smaller than the droplet thickness. Background parasitic signal can also be removed by two-photon absorption. In this case the measured fluorescence is concentrated at the focal point. The probe volume is very small, typically less than $1 \mu \mathrm{m}$ side [92].

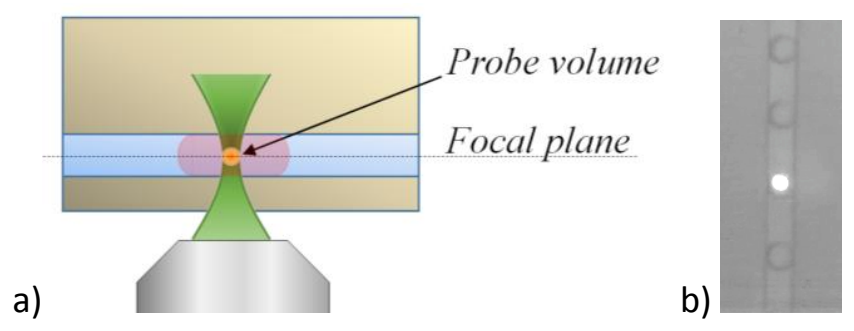

Fig. 2.46 a) Schematics of focused green laser spot in a droplet emitting orange fluorescence, b) top view image of a spot

Small probe volume can be required for Single Molecule Detection (SMD). It is often achieved with Spatial Two-Photon Fluorescence Cross-Correlation Spectroscopy [93]. However microfluidics offers the advantage to perform SMD through dilution without using an ultra-small probe volume. It benefits from the high repeatability on a large number of droplets in a small time that makes possible good statistical analysis [94]. Integrated fiber optics (or optical waveguides) may also improve the spatial resolution but it is limited by 
focusing issue at the blunt end of the fiber if there is no special ending [95-97]. Integrating spherical lenses to benefit from photonic nanojet is effective $[98,99]$. However these integrations require major changes in the process of fabrication of the chip.

Unless it is required, having a too small probe volume can be problematic. If the droplet content is inhomogeneous there is a risk that the fluorescent target is missed or in a region where the incident light is different (Fig. 2.456). This is a strong source of discrepancy for cell analysis in droplets for example. To avoid this, the light can be spread on a fine line across the canal width [100].

Other parameters to consider for fluorescence analysis in microfluidics are the $\mathrm{pH}$ (fluorophore are very sensitive to $\mathrm{pH}$ and a buffer is required), temperature (less sensitive), the formation of "excimer" with some fluorophores at high concentration. The latter causes a change of the fluorescence spectrum because of a change in the fluorophore nature. The biochemistry should take into account the environment of the fluorophore including the surfactant if there is any in the droplet. It is preferable to use a surfactant dissolved in the oil unless the surfactant is part of the biochemistry. For example, the surfactant is functionalized in [101]. With microfluidics, canal edges can also be problematic because the reflection of the laser beam on it can cause cross coupling in multiple points measurement for example. Another source of coupling is the diffusion of the fluorophores through the oil [102]. It is fortunately typically slow and depends strongly on the fluorophore, the liquids and the surfactant.

Sometimes, the biochemistry method needs a functionalized surface. Without modifying the process to functionalize the microfluidics chips it is possible to use functionalized magnetic bead. They are commercially available.

\subsubsection{Setup for fluorescence spectroscopy}

The figure 2.47 shows the optical setup for fluorescence spectroscopy with two static excitation monochromatic light sources that can be used simultaneously: blue $\left(\lambda_{0}\right)$ and green $\left(\lambda_{1}\right)$. Collimated laser sources suits very well for the application. Preferably they should be stable (power regulated if using laser diodes) because the measurement is sensitive to intensity. This setup also offers the possibility to read the fluorescence signal on two bands of interest: yellow with sensor $S_{0}$ and orange with sensor $S_{1}$. Each sensor measures the intensity over a certain time. This time can be long for a static observation in a microfluidics chamber. As the kinetic of the reactions are relatively slow, it is possible to average to obtain a good signal to noise ratio. For high throughput droplet analysis higher sensor bandwidth is required while the period of observation is shorter. Photomultiplier Tubes (PMT) are well suited in terms of performances (sensitivity, resolution, bandwidth, quantum yield). Furthermore the controlled amplification is adjusted to the signal level on a large dynamic range. The scintillator of the PMT should exhibit a good quantum yield at the considered wavelength. Alternatively Avalanche Photodiodes (APD) may also offer good performances [103]. 


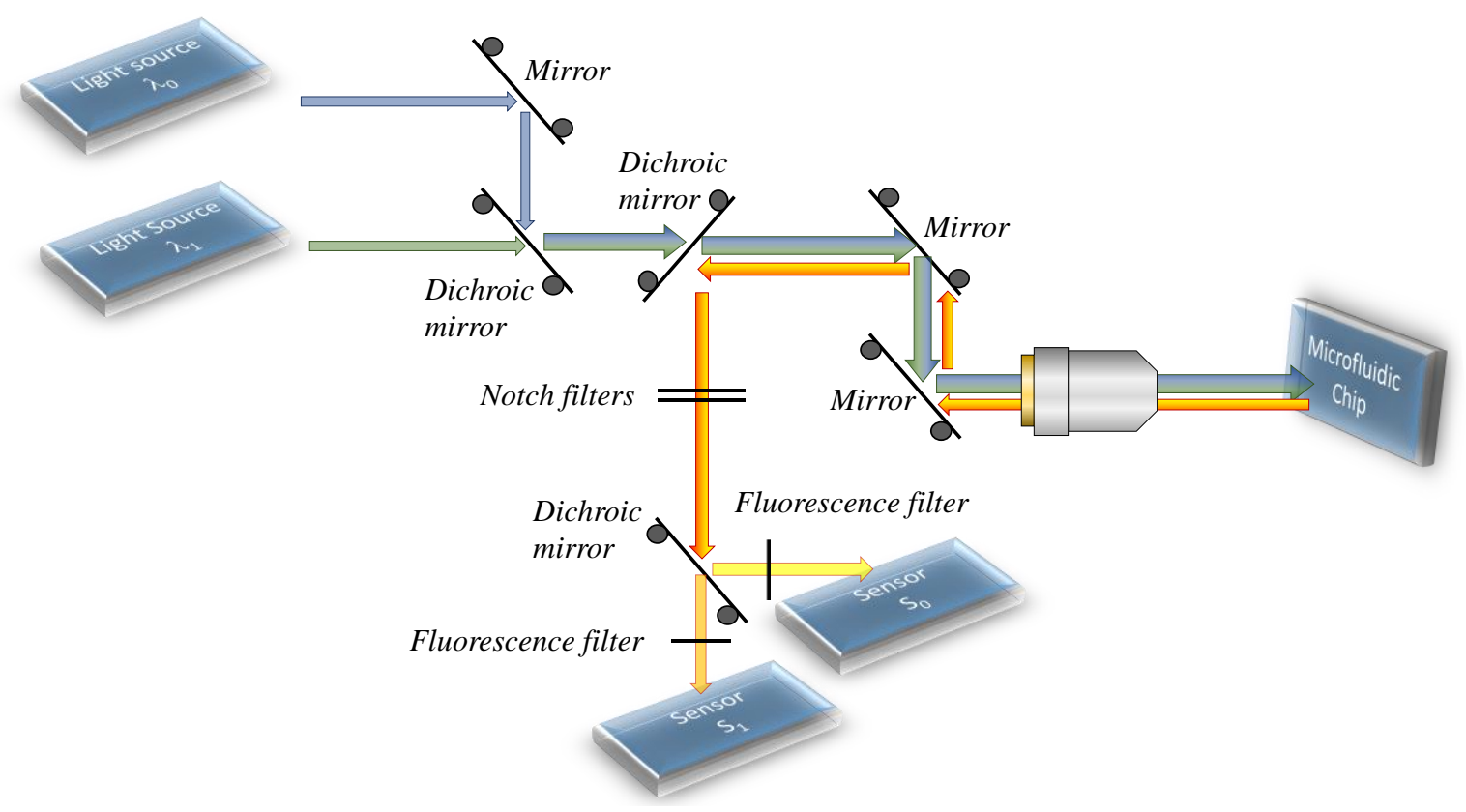

Fig. 2.47 fluorescence spectroscopy with two wavelength

The two light sources are combined in a single beam with a dichroic mirror which transmits the green light $\left(\lambda_{1}\right)$ without deflection and reflects the blue one in a specular way. The transition from transmission to reflectivity of this kind of mirror is quite sharp because it is based on interference with thin film layer. The mirror on the blue $\left(\lambda_{0}\right)$ source of light makes a $90^{\circ}$ turn to the beam but its main purpose is to offer a convenient way to adjust the blue beam spot on the dichroic mirror onto the outcoming spot of the transmitted green beam $\left(\lambda_{1}\right)$. It is preferable to have two fine knobs (tip and tilt, grey circle in figure 2.47) to adjust the orientation of the mirror plane and therefore the orientation of the laser beam and where it will hit the dichroic mirror. It should be noted that two degrees of freedom are enough to fully define the orientation of a beam. However, having two superposed spot on the dichroic mirror is not enough because the two beams can separate again. The two fine adjustment screws of the dichroic mirror will ensure that the beams are superposed and will hit the microfluidic chip at the same location. In theory, having two nonsuperposed laser beams hitting the chips at the same location is possible, if they are not too far from each other, but in practice superposition is recommended.

After passing through another dichroic mirror, the superposed beam reaches a telescopic system of two mirrors to adjust this beam on the axis of the objective. Without these 4 degrees of adjustment the position and orientation of the green laser would be necessary and it is much less convenient. The telescopic system enables also to change the direction of the beam if this setup is to be adapted on an inverted epifluorescence microscope for example. The objective can be the one of an epifluorescence microscope whether it is implemented in a microscope or not. If not mounted of a microscope, the objective or the chip should be placed on a translator to finely place the focal plane on the sample location in the chip. 2D translation of the chip in the focal place is also necessary to precisely hit the sample at its location. The size of the beam spot on the microfluidics chip is proportional to the wavelength, to the focal distance and inversely proportional to the initial beam diameter. These relations come from the limit of diffraction. For example if the spot size is $200 \mu \mathrm{m}$ with a focal distance of $12 \mathrm{~mm}$ and it will be $20 \mu \mathrm{m}$ for $1,2 \mathrm{~mm}$. A good numerical aperture will collect the maximum light but it requires a short focal distance for a given objective lens diameter. The microfluidics canal wall thickness will limit the focal distance.

The collected light is collimated when coming out of the objective. When reaching the first dichroic mirror on its path, it is reflected. The cutoff wavelength of this dichroic mirror should be between the excitation and the emission. However a small but still significant part of the laser beam, which comes from a reflection on the microfluidics chip, is also reflected by the dichroic mirror. It should be noted that dichroic 
mirror have a typical transmission coefficient of $90 \%$. It means that $10 \%$ is reflected even for a wavelength inside the transmission band (it is normally a non-dissipative element). Two sharp notch filters of high attenuation order (typically 5 or more) are used to remove efficiently the remaining excitation signals. Another dichroic mirror is used to separate the two bands of wavelength (yellow and orange on the example of the figure 2.47). A fluorescence filter is used to further filter what is outside the band considered (e.g. environmental light of the room). These filters are also based on interference in order to be very selective. In case that it is not sufficient and that differential measurement cannot be used (no droplet), it is possible to use a classical scheme with modulated light source and a lock-in amplifier to read out the signals.

Based on the same principle, the presented setup can be adapted for a single or more excitation light source and a single or more optical sensor. In practice, it is more convenient to measure the fluorescence while observing the microfluidics chip. For example it is possible to see where the laser spot hits the chip while making sure that droplet are properly formed in a canal. A white source of light (e.g. halogen lamp) is filtered and lightens the part of the chip to be observed (see figure 2.48). The filter is either high pass, low pass or band pass depending on where is a window of wavelength available. This light should not excite the fluorophores but also should not be measured as a fluorescence signal by the sensors. A dichroic mirror is used to separate the image (toward the camera and its optics) from the fluorescence signal. In an alternative configuration to the figure 2.48 setup, the image can go straight and the rest is reflected. A part of the fluorescence signal can also go toward the camera to have an image of the spot superposed with the image of the canal. Inverted microscope can be featured with wheels to hold and select the filter or the dichroic mirror so that the setup is adaptable. A safety filter should be added before the binocular to cutoff the excitation wavelength because reflection in the eyes is a risk.

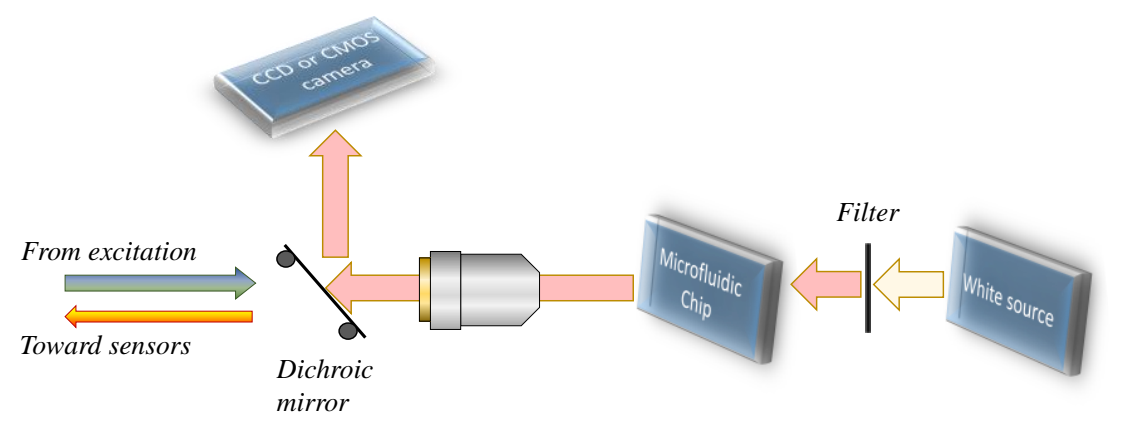

Fig. 2.48 : Additional camera setup

\subsubsection{Setup for fluorescence polarization}

To measure polarization some elements must be added to the setup of presented in figure 2.47. First, the light sources should be polarized. Lasers are usually polarized but a polarization filter can be added to keep the desired component of polarization. On the sensing side a polarization cube separates the two components (see figure 2.49). A polarization filter with the proper orientation is added before each sensor to further remove the unwanted component of the light. Finally the measurement is performed by calculating the polarization $(P)$ or the anisotropy $(r)$ from the intensity $\left(I_{/ /}\right.$and $\left.I_{\perp}\right)$ measured by the two sensors:

$$
\begin{aligned}
& P=\frac{I_{/ /}-I_{\perp}}{I_{/ /}+I_{\perp}} \\
& r=\frac{I_{/ /}-I_{\perp}}{I_{/ /}+2 \cdot I_{\perp}}
\end{aligned}
$$


Both values are null if the two components are equal. This is the case for non-polarized light. Because it is ratiometric, these two values do not depend on the global intensity level. It influences the signal to noise ratio but not the average value. For this reason polarization results are reproducible with different equipment whereas intensity measurement is used only to monitor change unless a reference sample is used. It is less sensitive to the light source stability and to all parameters of equation 1 (concentration, thickness...).

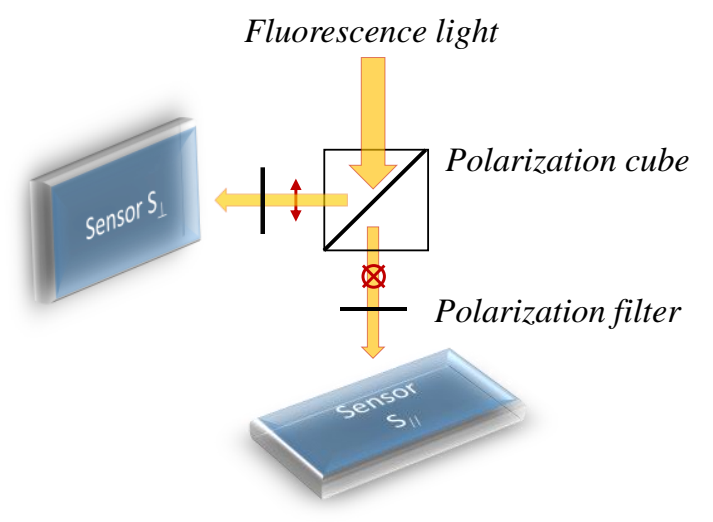

Fig. 2.49 Additional setup for polarization

\subsubsection{Setup for time resolved fluorescence}

Implementing time-resolved fluorescence does not modify the optical setup of figure 2.47. The light of source must be pulsed (see figure 2.50) at a rate higher than the droplet rate but smaller than at least 5 times the lifetime to measure. Pulse duration should be much smaller than the lifetime of the fluorophore (typically some ns). The pulse duration of laser diodes can be in the order of hundreds of ps [104]. It makes is suitable for many fluorophores. Many lasers offer better performance in terms of pulse duration or power but are more expensive and sometimes very long to stabilize.
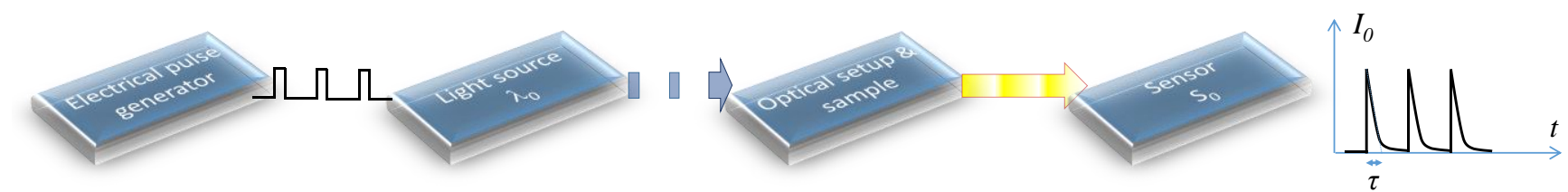

Fig. 2.50 Implementation for time resolved fluorescence spectroscopy

For the implementation presented on figure 2.50, the sensor must have a high bandwidth. The lifetime $\tau$ is extracted from the exponential decay contained in the sensor signal. Another way to obtain the exponential decay is called Time Correlated Single Photon Counting (TCSPC). It consists in measuring the arrival time of each single photon after a pulse with a Time to Digital Converter (TDC). The resolution of the TDC is in the order of the lifetime of smaller. The histogram of these arrival times is the exponential decay from which the lifetime is extracted. Single photon counting offers very good sensitivity. However, it requires that no more than one photon is received after a pulse. Otherwise there is a risk that two photons arrive too close. The second would fall into the dead time of the photon detector and would not be detected. As the photon emission is a Poisson process the probability is never null but for 1 photon over 10 pulses the lifetime estimation is quite accurate (3\%, [105]). This is reserved for low intensity. It has been shown to be applicable, using Single Photon Avalanche Diodes (SPAD) as detectors, for droplet analysis [106] at about 1000 droplets per second. The standard deviation on the lifetime measurement is limited by the quantic noise and is $1 / \sqrt{N}, \mathrm{~N}$ being the total number of received photon. Similarly to polarization, although it offers different possibilities, time resolved spectroscopy does not depend on the global intensity 
level. Intensity only affects signal to noise ratio. Therefore it is less sensitive to parameters such as light source stability and drift, concentration or thickness of the canal.

\subsubsection{2D imaging}

The setups for a single point measurement can be reused for $2 \mathrm{D}$ imaging. The sensor is replaced by a camera. There is a tradeoff of performance and cost for the choice of the camera: CCD, ICCD, EMCCD, CMOS, which can be cooled to enhance signal to electronic noise. The light source is less focused and often needs to be homogeneous on the area of observation. Although the performance are lower in terms of sensitivity, resolution, bandwidth on a single point (excitation light is not focused, each element of the sensing matrix is not optimized) it can be used to replace single point measurement and give supplementary information (e.g. the size and shape of a cell). It is possible to monitor cells labelled with fluorophore through time lapse microscopy in microfluidic culture [107]. To further improve the possibilities or the performances, confocal and two photons techniques are used. Dark field illumination also offers better performances because when the light comes from the sides there is less background signal [108]. On the sensor side, the intensity is not the only technique for imaging; time resolved fluorescence can be implemented with camera. This is called Fluorescence Lifetime Imaging (FLIM) [109].

\subsubsection{Wavelength-resolved spectroscopy and Raman scattering}

Using three color cameras without fluorescence filter offers some capabilities for wavelength resolved imagery. It can be used for barcoding droplets. This is useful to identify the droplet that is analyzed, particularly in high throughput application [110]. However the spectral resolution is too poor for many applications. Using several sensors with different fluorescence filters as in figure 2.47 is better but the number of sensors is limited. A prism or a monochromator will achieve better spectral resolution. Due to dispersion a spot of light is transformed in a 1D line of light. Each sensors put on this 1D line measured the intensity at a different wavelength (figure 2.51). If the fluorescence comes from a $1 \mathrm{D}$ line, the result is a $2 \mathrm{D}$ surface to be measured with a camera. One dimension is the spectral resolution and the other is the spatial resolution. An alternative is to use one dimension for spectral resolution and the second dimension for time resolution with a streak camera [111].

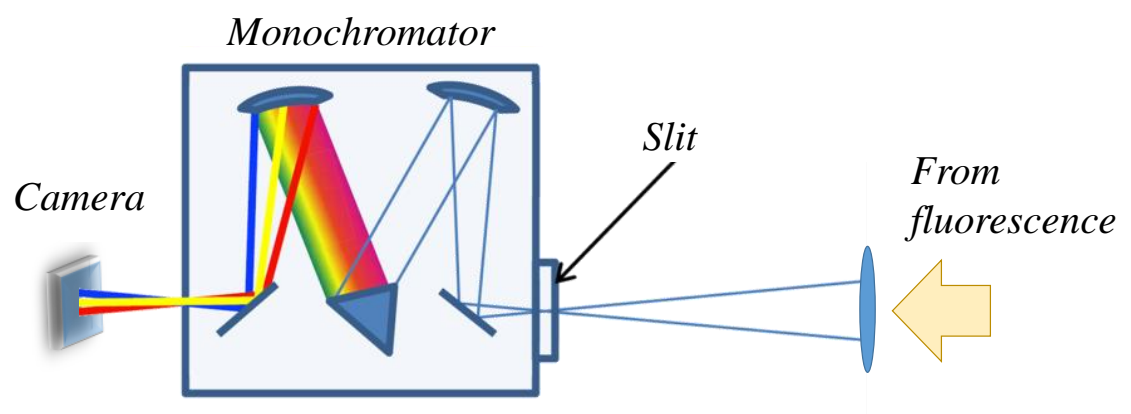

Fig. 2.51: Principle of spectrum decomposition using a monochromator

Raman scattering spectroscopy demands a very good resolution for distinguishing the emission peaks that characterize the sample. The Raman scattering is the absorption of a photon by a material and reemission at a lower wavelength (anti-stockes) because the material has lost energy. The spectrum of the emitted signal contains peaks that are a fingerprint of its chemical nature and its surrounding. It can be measured with a setup similar to the fluorescence spectroscopy. However the Raman diffusion is too low and mixed with other phenomenon (e.g. Mie and Rayleigh diffusion). Surface Enhanced Raman Spectroscopy (SERS) provides enough intensity for analysis in a microfluidic chip [112]. This technique requires a metal surface close to the sample. Metal deposition in a chamber works but the chip process has to be modified. Alternatively, using metal nanoparticle does not necessitate metal deposition. The nanotubes are suspended in a colloid solution and should be mixed very efficiently with the sample. This mixing can be 
done in the microfluidic chip. The main difficulty is to preserve the high SERS activity of the nanoparticle. To solve this problem other chemical substances have to be mixed.

\subsubsection{High throughput applications and cytometry}

High throughput screening is used for evaluating quickly the potential of very large number of molecules to have an interaction with a target (e.g. a cell). The information is mostly binary. If there is an interaction the molecule may be potentially a drug and is further study with other techniques. Robotic equipment working with microplate array intensively used to accelerate the test and reduced the quantity of chemicals per test. However, volumes that are smaller than microliter are very difficult to manipulate and the throughput is limited by the mechanical speed of the robot and parallelization. Microfluidics overcomes the difficulty of manipulating small volumes as a $100 \mathrm{pL}$ or smaller droplet is a routine with this technology. The droplet rate offers much higher throughput than robot capabilities. On one hand, there is still a difficulty for injecting the large number of products in a sequential way in the chip. On the other hand, it is easy to dilute an injected product to test it at different concentrations and obtain dose response at high speed. It been implemented with fluorescence spectroscopy in [113]. As some products react only for a certain range of concentration, this prevents false negative test.

Flow cytometry consists in counting the number of cells in a solution flow and to characterize it. Based on the measured characteristics the cells are sometimes sorted individually. The solution is injected in a capillary and the cells are passing in the probe volume one by one because of the diameter of the capillary force the cell to be aligned in a single line. If a light illuminates one side of the capillary, there is an intensity drop on the other side when a cell is passing by. A small microfluidic canal can replace the capillary. Sometimes, a sheath liquid is injected with a hydrodynamic focusing to run laminarly on the side of the sample solution and further reduce the width and forcing cell alignment. Fluorescence spectroscopy offers better sensitivity and more information. The laser spot size is important to distinguish between two close cells when they are in a continuous flow. If they are encapsulated in a droplet, special care should be taken to not encapsulate two cells in the same droplet. If a sorter is added the techniques is called FACS (Fluorescence Activated Cell Sorting). The same principle can be used for droplet sorting and is not limited to cell sorting [114].

\section{References}

[1] L. Gervais, N. de Rooij, E. Delamarche, Microfluidic Chips for Point-of-Care Immunodiagnostics, Adv. Mater. 23 (24), H151 (2011)

[2] C. D. Chin, V. Linder, S. K. Sia, Commercialization of microfluidic point-of-care diagnostic devices, Lab Chip 12, 2118 (2012)

[3] B. H. Weigl, R. L. Bardell, C. R. Cabrera, Lab-on-a-chip for drug development, Adv. Drug Deliv. Rev. 55(3), 349 (2003)

[4] J. Ducrée, S. Haeberle, S. Lutz, S. Pausch, F. Von Stetten, . Zengerle, The centrifugal microfluidic BioDisk platform, J. Micromech. Microeng. 17(7), S103 (2007)

[5] S.-Y. Teh, R. Lin, L.-H. Hung, and A. P. Lee, Droplet microfluidics, Lab Chip 8(2), 198 (2008)

[6] A. W. Martinez, S. T. Phillips, G. M. Whitesides, E. Carrilho, Diagnostics for the Developing World: Microfluidic Paper-Based Analytical Devices, Anal. Chem. 82(1), 3 (2010)

[7] T. M. Squires and S. R. Quake, Microfluidics: Fluid physics at the nanoliter scale, Rev. Mod. Phys. 77(3), 977 (2005) 
[8] A. Ghosh, Scaling Laws, in Mechanics Over Micro and Nano Scales, ed. by S. Chakraborty Springer (New York, 2011) p. 61

[9] D. J. Beebe, G. A Mensing, G. M. Walker, Physics and applications of microfluidics in biology, Annu. Rev. Biomed. Eng. 4, 261 (2002)

[10] F. M. White, I. Corfield, Viscous fluid flow 3 (McGraw-Hill, New York, 2006)

[11] J. S. Kuo, D. T. Chiu, Controlling Mass Transport in Microfluidic Devices, Annu. Rev. Anal. Chem., 4(1), 275 (2011)

[12] N. L. Jeon, S. K. W. Dertinger, D. T. Chiu, I. S. Choi, A. D. Stroock, G. M. Whitesides, Generation of solution and surface gradients using microfluidic systems, Langmuir 16(22), 8311 (2000)

[13] M. H. V Werts, V. Raimbault, R. Texier-Picard, R. Poizat, O. Français, L. Griscom, J. R. G. Navarro, Quantitative full-colour transmitted light microscopy and dyes for concentration mapping and measurement of diffusion coefficients in microfluidic architectures, Lab Chip 12(4), 808 (2012)

[14] M. H. V Werts, V. Raimbault, M. Loumaigne, L. Griscom, O. Français, J. R. G. Navarro, A. Débarre, B. Le Pioufle, Optical microscopy and spectroscopy of analyte-sensitive functionalized gold nanoparticles in microfluidic systems, in Colloidal Nanocrystals for biomedical applications VIII, Proc. SPIE 8595, 85950W (2013)

[15] T. P. Lagus, J. F. Edd, A review of the theory, methods and recent applications of high-throughput single-cell droplet microfluidics, J. Phys. D. Appl. Phys 46(11), 114005 (2013)

[16] H. Gu, M. H. G. Duits, F. Mugele, Droplets formation and merging in two-phase flow microfluidics, Int. J. Mol. Sci. 12(4), 2572 (2011)

[17] K. W. Oh, K. Lee, B. Ahn, E. P. Furlani, Design of pressure-driven microfluidic networks using electric circuit analogy, Lab Chip 12(3), 515 (2012)

[18] N. A. Mortensen, F. Okkels, H. Bruus, Reexamination of Hagen-Poiseuille flow: Shape dependence of the hydraulic resistance in microchannels, Phys. Rev. E 71(5), 57301 (2005)

[19] T. Bourouina, A. Bosseboeuf, and J.-P. Grandchamp, Design and simulation of an electrostatic micropump for drugdelivery applications, J. Micromech. Microeng. 7(3), 186 (1997)

[20] M. A. Holden, S. Kumar, E. T. Castellana, A. Beskok, P. S. Cremer, Generating fixed concentration arrays in a microfluidic device, Sensors \& Actuators, B Chem. 92(1-2), 199 (2003)

[21] Y. Wang, T. Mukherjee, Q. Lin, Systematic modeling of microfluidic concentration gradient generators," J. Micromech. Microeng. 16(10), 2128 (2006)

[22] N. Li Jeon, H. Baskaran, S. K. W. Dertinger, G. M. Whitesides, L. Van de Water, M. Toner, Neutrophil chemotaxis in linear and complex gradients of interleukin8 formed in a microfabricated device, Nat. Biotechnol. 20(8), 826 (2002)

[23] J. P. Brody, P. Yager, Diffusion-based extraction in a microfabricated device, Sensors \& Actuators A Phys. 58(1), 13 (1997) 
[24] P. Yager, T. Edwards, E. Fu, K. Helton, K. Nelson, M. R. Tam, B. H. Weigl, Microfluidic diagnostic technologies for global public health, Nature 442(7101), 412 (2006)

[25] T. Schneider, J. Kreutz, D. T. Chiu, The Potential Impact of Droplet Microfluidics in Biology, Anal. Chem. 85(7), 3476 (2013)

[26] X. Casadevall i Solvas, A. deMello, Droplet microfluidics: recent developments and future applications., Chem. Commun. 47 (7), 1936 (2011)

[27] T. Thorsen, R. W. Roberts, F. H. Arnold, and S. R. Quake, Dynamic Pattern Formation in a VesicleGenerating Microfluidic Device, Phys. Rev. Lett. 86(18), 4163 (2001)

[28] S. L. Anna, N. Bontoux, H. A. Stone, Formation of dispersions using 'flow focusing' in microchannels, Appl. Phys. Lett. 82(3), 364 (2003)

[29] R. Seemann, M. Brinkmann, T. Pfohl, and S. Herminghaus, Droplet based microfluidics, Reports Prog. Phys. 75(1), 16601 (2012)

[30] R. Gorkin, J.-M. J. Park, J. Siegrist, M. Amasia, B. S. Lee, J.-M. J. Park, J. Kim, H. Kim, M. Madou, Y.-K. Cho, Centrifugal microfluidics for biomedical applications, Lab Chip 10(14), 1758 (2010)

[31] J. Ducrée, S. Haeberle, T. Brenner, T. Glatzel, R. Zengerle, Patterning of flow and mixing in rotating radial microchannels, Microfluid. Nanofluidics 2(2), 97 (2006)

[32] M. Madou, J. Zoval, G. Jia, H. Kido, J. Kim, N. Kim, "Lab on a CD," Annu. Rev. Biomed. Eng. 8(1), 601 (2006)

[33] O. Strohmeier, M. Keller, F. Schwemmer, S. Zehnle, D. Mark, F. von Stetten, R. Zengerle, N. Paust, Centrifugal microfluidic platforms: advanced unit operations and applications, Chem. Soc. Rev. 44(17), 6187 (2015)

[34] A. W. Martinez, S. T. Phillips, M. J. Butte, G. M. Whitesides, Patterned Paper as a Platform for Inexpensive, Low-Volume, Portable Bioassays, Angew. Chemie Int. Ed. 46(8), 1318 (2007)

[35] Y. Xia, J. Si, Z. Li, Fabrication techniques for microfluidic paper-based analytical devices and their applications for biological testing: A review, Biosens. Bioelectron. 77, 774 (2016)

[36] A. K. Yetisen, M. S. Akram, C. R. Lowe, Paper-based microfluidic point-of-care diagnostic devices, Lab Chip 13(12), 2210 (2013)

[37] J.M. Berg, J.L. Tymoczoko, G.J. Gatto, L ; Stryer, "Biochemistry", 8th edn. (W.H. Freeman and Co Ltd, New York, 2015)

[38] M. D. Hanwell, D. E. Curtis, D. C. Lonie, T. Vandermeersch, E. Zurek, G. Hutchinson, Avogadro: an advanced semantic chemical editor, visualization, and analysis platform, J. Cheminformatics 4, 17 (2012).

[39] T.J. Smith, MOLView: A program for analyzing and displaying atomic structures on the Macintosh personal computer, J. Molecular Graphics 13(2), 1995. Website: http://molview.org/. Accessed 3 April 2018 
[40] [The PyMOL Molecular Graphics System, Version 1.8 Schrödinger, LLC. Website: http://pymol.org. Accessed 3 April 2018

[41] E.T. Bolton, J. McCarthy, A General Method for the Isolation of RNA complementary to DNA, in Proc. Natl. Acad. Sci. USA 48(8), 1390 (1962)

[42] A.H. Sturtevant, A History of Genetics, (Cold Spring Harbor Laboratory Press, 2001)

[43] A. Abbas, A.H. Lichtman, S. Pillai, Cellular and Molecular Immunology, 9th edn.(Elsevier, Philadelphia,2018)

[44] X.L. Su, Y. Li, A self-assembled monolayer-based piezoelectric immunosensor for rapid detection of Escherichia coli 0157:H7, Biosensors and Biolectronics 19(6), 563 (2004)

[45] C. Prussin, D.D. Metcalfe, Detection of intracytoplasmic cytokine using flow cytometry and directly conjugated anti-cytokine antibodies, J. Immunol. Methods 188(1), 117 (1995)

[46] B.J. Takács, Protein Purification: Theoretical and Methodological Considerations, in Encyclopedia of Analytical Chemistry : Applications, Theory and Instrumentation (Wiley \& Sons Ltd, 2006)

[47] J. Gao, S. Ma, D.T. Major, K. Nam, J. Pu, D.G. Truhlar, Mechanisms and Free Energies of Enzymatic Reactions, Chem. Rev. 106(8), 3188 (2006)

[48] D. Melloul, S. Marshak, E. Cerasi, Regulation of insulin gene transcription, Diabetologia, 45(3), 309 (2012)

[49] F. Jacob, J. Monod, Genetic regulatory mechanisms in the synthesis of proteins, J. Molecular Biology 3, 318 (1961)

[50] S. Hoops, S. Sahle, R. Cauges, C. Lee, J. Pahle, N. Simus, M. Singhal, L. Xu, P. Mendes, U. Kummer, COPASI - a Complex Pathway Simulator, Bioinformatics 22(24), 3067 (2006)

[51] Y. Gendrault, M. Madec, C. Lallement, J. Haiech, Modeling biology with HDL languages: a first step toward a genetic design automation tool inspired from microelectronics, IEEE Trans. Biomed. Eng. 61(4), $1231(2014)$

[52] J. Haiech, Y. Gendrault, M.C. Kilhoffer, R. Randjeva, M. Madec, C. Lallement, A general framework improving teaching ligand binding to a macromolecule, Biochim. BioPhys. Acta, 1843(10), 2348 (2014)

[53] J.N. Weiss, The Hill equation revisited : uses and misuses, the FASEB Journal 11(11), 835 (1997)

[54] U. Alon, An introduction to systems biology : Design principles of biological circuits, (Chapman \& Hall/CRC, 2006)

[55] J.H. Santiago, S.T. Wereley, C.D. Meinhart, D.J. Beebe, R.J. Adrian, A particle image velocimetry system for microfluidics, Exp. in Fluids 25(4), 316 (1998)

[56] S.T. Wereley, J.G. Santiago, R. Chiu, C.D. Meinhart, RJ. Adrian, Micro-resolution particle image velocimetry, in Proc Micro- and nanofabricated structure s and devices for biomedical environment applications, Proc. SPIE 3258, 122 (1998) 
[57] C. Cierpa, C. J. Kähler, Particle imaging techniques for volumetric three-component (3D3C) velocity measurements in microfluidics, J. Vis, 15, 1 (2012)

[58] S.T. Wereley, CD. Meinhart Recent advances in Micro-Particle Image Velocimetry, Annu. Rev. Fluid Mech. 42, 557 (2010)

[59] H. F. Li , M. Yoda, Multilayer nano-particle image velocimetry (MnPIV) in microscal Poiseille flows, Meas. Sci. Technol. 19, 075402 (2008)

[60] S.L. Anna, Droplets and bubbles in microfluidic devices, Annu. Rev. Fluid Mech 48, 285 (2016)

[61] R. Seemann, M. Brinkmann, T. Pfohl, S. Herminghaus, Droplet based microfluidics, Rep. Prog. Phys. 75016601 (2012)

[62] M. Rossi, R. Lindken, BP. Hierck, J. Westerweel, Tapered microfluidic chip for the study of biochemical response at subcellular level of endothelial cell to shear flow, Lab on a Chip, 9, 1403 (2009)

[63] L.E. Rodd, J.J. Cooper-White, D.V. Boger, GH. McKinley, Role of the elasticity number in the entry flow of dilute polymer solutions in micro-fabricate contraction geometries, J. Non-Newtonian Fluid Mech. 143, 170191 (2007)

[64] J. Rumble (ed.),CRC Handbook of Chemistry and Physics, $98^{\text {th }}$ edn. (Taylor \& Francis Group, 2017)

[65] C. W. Macosko. Rheology: Principle, Measurements, and Application, (WILEY-VCH, 1994)

[66] G. Astarita, G. Marrucci, L. Nicolais (ed), Rheology, Volume 1 : Principles, (Springer,1980)

[67] H. A. Barnes, J. F. Hutton, K. Walters, Vol.3: An introduction to rheology, $3^{\text {rd }}$ edn. (Elsevier,AmsterdamLondon-New York-Tokyo, 1993)

[68] P.-G. De Gennes, F. Brochard-Wyart, D. Guere, Capillary and Wetting Phenomena -Drops, Bubbles, Pearls, Waves, (Springer, New York, 2004)

[69] C.D. Meinhart, S.T. Wereley, M.H.B. Gray, Volume illumination for two-dimensional particle image velocimetry, Meas. Sci. Technol. 11:809 (2000)

[70] C.J. Bourdon, M.G. Olsen, A.D. Gorby, Validation of an analytical solution for depth of correlation in microscopic particle image velocimetry, Meas. Sci. Technol. 15, 318 (2004)

[71] M.G. Olsen, R. J. Adrian, Out-of-focus effects on particle image visibility and correlation in microscopic particle image velocimetry, Exp. Fluids, 29 S166 (2000)

[72] O. Carrier, F.G. Ergin, H.Z. Li, B.B. Watz, D. Funfschilling, Time-resolved mixing and flow-field measurements during droplet formation in a flow-focusing junction, J. Micromech. Microeng. 25, 084014 (2015)

[73] S. Devasenathipathy, J. G. Santiago, S. T. Wereley, C. D. Meinhart, K. Takehara, Particle imaging techniques for microfabricated fluidic systems, Experiments in Fluids, 34, 504 (2003)

[74] V. Van Steijn, MT. Kreutzer, CR. Kleijn, $\mu$-PIV study of the formation of segmented flow in microfluidic Tjunctions, Chem. Eng. Science 62, 7505 (2007)

[75] D. Funfschilling, H. Debas, H.Z. Li, T.G. Mason, Flow-field dynamics during droplet formation by dripping in hydrodynamic-focusing microfluidics, Phys. Rev. E 80, 015301 (2009)

[76] D.E. Smith, S. Chu, Response of flexible polymers to sudden elongational flow, Science 281, 1335 (1998)

[77] L. Boussel, V. Rayz, C. McCulloch, A. Martin, G. Acevedo-Bolton, M. Lawton, R. Higashida, W. S. Smith, W. L. Young, D.Sloner, Aneurysm growth occurs at region of low wall shear stress, Stroke, 39, 2997 (2008) 
[78] J. N. Tropper, M. A. Gimbrone, Blood flow and vascular gene expression: fluid shear stress as a modulator of endothelial phenotype, Molecular Medicine Today, 5, 40 (1999)

[79] C. Poelma, K. Van der Heiden, B. P. Hierck, R. E. Poelmann, J. Wersterweel, Measurements of the wall shear stress distribution in the outflow track of an embryonic chicken heart, J. R. Soc. Interface, 7, 91 (2010)

[80] C. Poelma, P. Vennemann, R. Lindken, J. Westerweel, In vivo blood flow and wall shear stress measurements in vitelline network, Exp. Fluids, 45, 703 (2008)

[81] Y. Sugii, R. Okuda, K. Okamoto, M. Takeda, Velocity measurement of both red blood cells and plasma of in vitro blood flow using high-speed micro PIV technique, Meas. Sci. Technol. 16, 1126 (2005)

[86] K. E. Audrey, T. P. Scott, C. S. Adam, A. M. Katherine, W. M. Andres, S. Pierre, J. Nina, P. Mara, M. W. George, Quantifying Colorimetric Assays in Paper-Based Microfluidic Devices by Measuring the Transmission of Light through Paper, Analytical Chemistry 81(120), 8447 (2009)

[82] M. O'Toole, D. Diamond, Absorbance Based Light Emitting Diode Optical Sensors and,sensing devices, Sensors 8, 2453 (2008)

[83] A. Hsiao, M. R. Gartia, T.-W. Chang, X. Wang, P. Khumwan, G. L. Liu, Colorimetric plasmon resonance microfluidics on nanohole array sensors, Sensing and Bio-Sensing Research 5, 24 (2015)

[84] M. T. Koesdjojo, S. Pengpumkiat, Y. Wu, A. Boonloed, D. Huynh, T. P. Remcho, A. V. T. Remcho, Cost Effective Paper-Based Colorimetric Microfluidic Devices and Mobile Phone Camera Readers for the Classroom, J. Chem. Educ. 92(4), 737 (2015)

[85] Y. Jung, J. Kim, O. Awofeso, H. Kim, F. Regnier, E. Bae, Smartphone-based colorimetric analysis for detection of saliva alcohol concentration, Appl. Opt. 54(131), 9183 (2015)

[86] G. Minas, J.C. Ribeiro, R.F. Wolffenbuttel, J.H. Correia, On-Chip Integrated CMOS Optical Detection Microsystem for Spectrophotometric Analyses in Biological Microfluidic Systems, in IEEE International Symposium on Industrial Electronics, Dubrovnik, Croatia, June 2005.

[87] Lakowicz, Joseph R., Principles of Fluorescence Spectroscopy, $3^{\text {rd }}$ Edn. (Springer, 2006)

[88] M. Kollner, J. Wolfrum, How many photon are necessary for fluorescence-lifetime measurements?, Chem. Phys. Lett. 200(12), 199 (1992)

[89] H. Geng, J. Feng,L. M. Stabryla, S. K. Cho, Dielectrowetting manipulation for digital microfluidics: creating, transporting, splitting, and merging of droplets, Lab Chip 17(6), 1060 (2017)

[90] A. Piruska, I. Nikcevic, S. H. Lee, C. Ahn, W. R. Heineman, P. A. Limbacha, C. J. Seliskar, The autofluorescence of plastic materials and chips measured under laser irradiation, Lab on a Chip 12, 1348(2005)

[91] G. D. M. Jeffries, R. M. Lorenz, D. T. Chiu, Ultrasensitive and High-Throughput Fluorescence Analysis of Droplet Contents with Orthogonal Line Confocal Excitation, Anal Chem. 82(23), 9948 (2010)

[92] Y Zeng, L. Jiang, W. Zheng, D. Li, S. Yao, J. Y. Qu, Quantitative imaging of mixing dynamics in microfluidic droplets using two-photon fluorescence lifetime imaging, Opt. Lett. 36, 2236- (2011). 
[93] P.S. Dittrich, P. Schwille, Spatial two-photon fluorescence crosscorrelation spectroscopy for controlling molecular transport in microfluidic structures", Anal Chem 74, 4472 (2002)

[94] A. M Streets, Y. Huang, Microfluidics for biological measurements with single-molecule resolution", Current Opinion in Biotechnology 25, 69 (2014)2014

[95] X. Su, S. E. Kirkwood, M. Gupta, L. Marquez-Curtis, Y. Qiu, A. Janowska-Wieczorek, W. Rozmus, Ying Y. Tsui, Microscope-based label-free microfluidic cytometry, Optics Express 19(1), 387- (2011)

[96] Y.-C. Tung, M. Zhang, C.-T. Lin, K. Kurabayashi, S. J. Skerlos, PDMS-based opto-fluidic micro flow cytometer with two-color, multi-angle fluorescence detection capability using PIN photodiodes, Sensors and Actuators B: Chemical 98(2-3), 356, (2004)

[97] M. L. Chabinyc, D. T. Chiu, J. C.McDonald, A. D. Stroock, J. F. Christian, A.M. Karger, and G. M. Whitesides, An Integrated Fluorescence Detection System in Poly(dimethylsiloxane) for Microfluidic Applications, Anal. Chem. 73, 4481 (2001)

[98] P. Ghenuche, J. de Torres, P. Ferrand, J. Wenger, Multi-focus parallel detection of fluorescent molecules at picomolar concentration with photonic nanojets arrays, Appl. Phys. Lett. 105, 131102 (2014)

[99] Y. J. Fan, Y. C. Wu, Y. Chen, Y. C. Kung, T. H. Wu et al., Three dimensional microfluidics with embedded microball lenses for parallel and high throughput multicolor fluorescence detection, Biomicrofluidics 7, 044121 (2013

[100] E. Schonbrun, P. E. Steinvurzel, K. B. Crozier, A microfluidic fluorescence measurement system using an astigmatic diffractive microlens array, Optics Express 19(2), 1385 (2011

[101] S. Ursuegui, M. Mosser, A. Wagner, Copper-free click chemistry for microdroplet's W/O interface engineering. RSC Advances, 6(97), 94942 (2016).

[102] P. Gruner, B. Riechers, B. Semin, J. Lim, A.Johnston, K. Short, J.-C. Baret, Controlling molecular transport in minimal emulsions, Nature Communications 7, 10392 (2016),.

[103] M. S.-Art, A. J. deMello, J.B. Edel, High-Throughput DNA Droplet Assays Using Picoliter Reactor Volumes, Anal. Chem. 79(17), 6682 (2007)

[104] W. Uhring, V. Zint, J. Bartringer, A low-cost high-repetition-rate picosecond laser diode pulse generator, in Semiconductor lasers and laser dynamics, Strasbourg,Sept.2004, Proc. SPIE 5452, 583 (2004)

[105] P. B. Coates, The correction for photon 'pile-up' in the measurement of radiative lifetime, J. Physics E: Sci. Instrum. 1(8), 878 (1968)

[106] J. Leonard, N. Dumas, J.P. Caussé, S. Maillot, N. Giannakopoulou, S. Barre, W. Uhring. Highthroughput time-correlated single photon counting, Lab-on-Chip 14(22), 4297 (2014)

[107] I. Santi, N. Dhar, D. Bousbaine, Y. Wakamoto, J. D. McKinney, Single-cell dynamics of the chromosome replication and cell division cycles in mycobacteria. Nat. commun. 4, 2470 (2013) 
[108] Y. Zhao, D. Chen, H. Yue, M. M. Spiering, C. Zhao, S. J. Benkovic, T. Jun Huang, Dark-Field Illumination on Zero-Mode Waveguide/Microfluidic Hybrid Chip Reveals T4 Replisomal Protein Interactions, Nano Lett. 14(4), 1952 (2014)

[109] G. Giraud, H. Schulze, D.-U. Li, T. T. Bachmann, J. Crain, D.Tyndall, J.Richardson, R.Walker, D. Stoppa, E. Charbon, R. Henderson, J. Arlt, Fluorescence lifetime biosensing with DNA microarrays and a CMOS-SPAD imager, Biomed. Opt. Express 1, 1302-1308 (2010)

[110] Y. Ding, S. Stavrakis, X. Casadevalli Solvas, A. J. deMello, A high throughput droplet-based microfluidic barcode generator, in 17th International Conference on Miniaturized Systems for Chemistry and Life Sciences, 27-31 October 2013, Freiburg, Germany.

[111] M. Zlatanski, W. Uhring, and J.-P. Le Normand, Sub-500-ps Temporal Resolution Streak-Mode Optical Sensor, IEEE Sensors Journal, 15(11), 6570 (2015)

[112] Q. Zhoua, T. Kima, Review of microfluidic approaches for surface-enhanced Raman scattering", Sensors and Actuators B: Chemical 227, 504 (2016)

[113] O. J. Miller, . El Harrak, T. Mangeat, J.-C. Baret, L. Frenz, B. El Debs, E. Mayot, M. L. Samuels, E. K. Rooney, P. Dieu, M. Galvan, D. R. Link, A. D. Griffiths, High-resolution dose-response screening using droplet-based microfluidics, Proc Natl Acad Sci U S A. 109(2): 378-383 (2002)

[114] J.-C. Baret, O. J. Miller, V. Taly, M. Ryckelynck, A. El-Harrak, L. Frenz, C. Rick, M. L. Samuels, J. B. Hutchison, J. J. Agresti, D. R. Link, D. A. Weitz, A. D. Griffiths,Fluorescence-activated droplet sorting (FADS): efficient microfluidic cell sorting based on enzymatic activity. Lab on a Chip, 9(13), 18501858 (2009) 